\title{
Warm gas in central regions of nearby galaxies
}

\section{Extended mapping of $\mathrm{CO}(3-2)$ emission}

\author{
M. Dumke ${ }^{1,2,3}$, Ch. Nieten ${ }^{2}$, G. Thuma ${ }^{2}$, R. Wielebinski ${ }^{2}$, and W. Walsh ${ }^{2}$ \\ 1 SMTO, Steward Observatory, The University of Arizona, 933 N. Cherry Avenue, Tucson, Arizona 85721, USA \\ 2 Max-Planck-Institut für Radioastronomie, Auf dem Hügel 69, 53121 Bonn, Germany \\ 3 Institut de Radio Astronomie Millimétrique, 300 rue de la Piscine, 38406 St. Martin d'Hères, France
}

Received 30 August 2000 / Accepted 3 May 2001

\begin{abstract}
We have mapped the $\mathrm{CO}(3-2)$ line emission from several nearby galaxies, using the Heinrich-HertzTelescope on Mt. Graham, Arizona. Unlike earlier observations, our investigation is not restricted to starburst galaxies, but includes twelve galaxies of various types and in different stages of star forming activity. Furthermore, we have not only observed the central positions of these objects, but have obtained maps of the extended $\mathrm{CO}(3-2)$ emission, with a typical map extent of 2 to 3 arcmin in each direction. Our observations show that this extended mapping is necessary to reveal spatial changes of the ISM properties within the galaxies. In this paper we present the data sets and some data analysis. We compare the galaxies in view of their morphology and excitation conditions, using line ratios, luminosities and other properties, like the extent of the $\mathrm{CO}(3-2)$ emission. The main results of this $\mathrm{CO}(3-2)$ survey are: 1 . In none of the observed objects the emission is confined to the nucleus, as claimed in some earlier publications. $\mathrm{CO}(3-2)$ emission can be detected for some objects to the same extent as the $\mathrm{CO}(2-1)$ and the $\mathrm{CO}(1-0)$ lines. 2. The emission is more concentrated to the vicinity of star forming structures (nuclear regions and spiral arms) than the lower CO transitions for most of the observed objects. This is shown by decreasing $(3-2) /(1-0)$ line intensity ratios from the very centres towards larger radii. The (deconvolved) sizes of the central emission peaks in the $\mathrm{CO}(3-2)$ line vary from about $300 \mathrm{pc}$ up to $3 \mathrm{kpc}$. 3 . The $\mathrm{CO}(3-2)$ luminosity is stronger in objects that contain a nuclear starburst or morphological peculiarities. The total power emitted in the $\mathrm{CO}(3-2)$ line from the central regions (i.e. excluding spiral arms/outer disk) is highest in the starburst galaxies NGC 2146, M 82, NGC 3628, and in the spiral galaxy M 51. When comparing the total power normalized to the size of the emission region, the starburst galaxies M 82 and NGC 253 show the highest values (about three times higher than most other objects), while NGC 278 and NGC 4631 show the lowest. 4. With the present spatial resolution, the line ratios $R_{3,1}$ seem to be independent of Hubble type, color or luminosity. Most galaxies with enhanced central star formation ("starbursts") show line ratios of the integrated intensities of $R_{3,1} \sim 1.3$ in the very centre and $\sim 1.0$ at a radius of about $1 \mathrm{kpc}$. Objects with a ring-like (or double-peak if seen edge-on) molecular gas distribution (NGC 253, M 82, and NGC 4631) show lower ratios. The two galaxies that have $\mathrm{CO}(3-$ 2) emission distributed over their spiral arms (NGC 891 and M 51) show very low line ratios despite their high infrared luminosities. This result suggests that CO emission in these objects reflects a large amount of molecular gas, but not enhanced star forming activity. 5. Starburst galaxies show $\mathrm{CO}(3-2)$ emission also in their disks. The line intensities are higher than that of normal galaxies. This suggests that even if a starburst is a localized phenomenon, it is related to different properties of the molecular gas over the whole galaxy.
\end{abstract}

Key words. galaxies: ISM - galaxies: spiral - radio lines: galaxies

\section{Introduction}

Lower lying $\mathrm{CO}$ line transitions which are observable in the mm-wavelength range are a widely used tracer of molecular hydrogen. Extensive work has been done for the $\mathrm{CO}(1-0)$ and the $\mathrm{CO}(2-1)$ line, whose upper levels are lying $5.5 \mathrm{~K}$ and $17 \mathrm{~K}$ above the ground level. Even if these

Send offprint requests to: M. Dumke, e-mail: mdumke@as.arizona.edu observations are a reliable tracer of the total column densities of $\mathrm{H}_{2}$ in external galaxies, where in general several molecular cloud complexes are covered by one telescope beam, these lines cannot trace $\mathrm{CO}$ gas which is highly excited. This higher excitation could be caused by collisional processes in a dense medium or by a strong radiation field leading to higher temperatures. Since these conditions often occur in the inner regions of molecular clouds where star formation is taking place, other tracers are needed 
to determine the physical state of the gas that actually is forming stars.

The $J=3$ level of the $\mathrm{CO}$ molecule lies $33 \mathrm{~K}$ above the ground state, and the critical density for the 3-2 transition is $\sim 5 \times 10^{4} \mathrm{~cm}^{-3}$. It is therefore only excited if the gas is sufficiently warm and/or dense. Whereas the lower transitions trace the large bulk of molecular gas at low and moderate temperatures, the $\mathrm{CO}(3-2)$ line gives some insight into the molecular gas in the immediate surroundings of star forming regions, where gas properties may be different.

While numerous publications exist about observations of the $\mathrm{CO}(1-0)$ and the $\mathrm{CO}(2-1)$ lines in nearby galaxies - large surveys in which only one or a few points in many galaxies have been observed (e.g. Braine et al. 1993; Young et al. 1995) as well as detailed mapping observations of selected objects (e.g. Reuter et al. 1991; García-Burillo et al. 1992; Golla \& Wielebinski 1994; and many others) - the number of published $\mathrm{CO}(3-2)$ observations is still small. The reason for this was a lack of good sub-mm telescopes on sites which allow observations at $345 \mathrm{GHz}$, the rest frequency of the $\mathrm{CO}(3-2)$ line. The first detection of extragalactic $\mathrm{CO}(3-2)$ was reported by Ho et al. (1987), in the nearby galaxy IC 342 .

In the following years, a few - mainly starburst galaxies have been investigated in the $\mathrm{CO}(3-2)$ line, but these observations usually covered only the very centre. Recently Mauersberger et al. (1999) observed a larger sample of about 30 nearby galaxies, but this was restricted to one position for each of these objects. Extended maps of three nearby galaxies were presented by Wielebinski et al. (1999).

In this paper we report the extensive mapping of 12 large nearby galaxies in the $\mathrm{CO}(3-2)$ line. The main goal of these observations was to obtain a homogeneous set of $\mathrm{CO}(3-2)$ data from a galaxy sample which covers various Hubble types as well as different stages of activity. While the spatial resolution of our observations is not sufficient to investigate the physical conditions in individual molecular clouds, the data provide information on the large-scale distribution of highly excited CO gas in galaxies. Together with published data from lower transitions it is possible to determine the global excitation state of the molecular gas as a function of star forming activity and the location of the emitting region in the observed objects.

The main criterion used to select the target galaxies was the strength of the $\mathrm{CO}(1-0)$ line. Further criteria were the expected global line-width (which should be small enough to fit into the limited bandwidth) and the availability of sufficiently large data sets of the lower CO transitions. In addition the visibility at the observatory site was considered for the selection. In Sect. 2 we describe the performed observations and the steps of reducing and calibrating the data, while in Sect. 3 we present the main results. This section includes also a set of figures for each object. We compare the observed galaxies in view of their morphology, kinematics, and excitation conditions
Table 1. Galaxies mapped in the $\mathrm{CO}(3-2)$ emission, with their adopted positions and LSR velocities. The coordinates given here represent the $(0,0)$ position in the maps shown in Sect. 3. References are: NGC 253, NGC 278, NGC 2146, NGC 4631, and NGC 6946 (Young et al. 1995), NGC 891 (García-Burillo et al. 1992), Maffei 2 (Weliachew et al. 1988), IC 342 (Ishizuki et al. 1990), M 82 (Rieke et al. 1980), NGC 3628 (Irwin \& Sofue 1996), M 51 (García-Burillo et al. 1993), and M 83 (Petitpas \& Wilson 1998).

\begin{tabular}{lrrc}
\hline Source & RA $(2000)$ & Dec. $(2000)$ & $v_{\text {lsr }}\left[\mathrm{km} \mathrm{s}^{-1}\right]$ \\
\hline NGC 253 & $0^{\mathrm{h}} 47^{\mathrm{m}} 33^{\mathrm{s}} .0$ & $-25^{\circ} 17^{\prime} 20^{\prime \prime}$ & 251 \\
NGC 278 & $0^{\mathrm{h}} 52^{\mathrm{m}} 04^{\mathrm{s}} .5$ & $47^{\circ} 33^{\prime} 01^{\prime \prime}$ & 640 \\
NGC 891 & $2^{\mathrm{h}} 22^{\mathrm{m}} 33^{\mathrm{s}} .1$ & $42^{\circ} 20^{\prime} 55^{\prime \prime}$ & 527 \\
Maffei 2 & $2^{\mathrm{h}} 41^{\mathrm{m}} 55.2$ & $59^{\circ} 36^{\prime} 11^{\prime \prime}$ & -36 \\
IC 342 & $3^{\mathrm{h}} 46^{\mathrm{m}} 48^{\mathrm{s}} .3$ & $68^{\circ} 05^{\prime} 46^{\prime \prime}$ & 32 \\
NGC 2146 & $6^{\mathrm{h}} 18^{\mathrm{m}} 37^{\mathrm{s}} .6$ & $78^{\circ} 21^{\prime} 19^{\prime \prime}$ & 903 \\
M 82 & $9^{\mathrm{h}} 55^{\mathrm{m}} 52^{\mathrm{s}} .6$ & $69^{\circ} 40^{\prime} 47^{\prime \prime}$ & 225 \\
NGC 3628 & $11^{\mathrm{h}} 20^{\mathrm{m}} 17^{\mathrm{s}} .0$ & $13^{\circ} 35^{\prime} 20^{\prime \prime}$ & 844 \\
NGC 4631 & $12^{\mathrm{h}} 42^{\mathrm{m}} 07^{\mathrm{s}} .7$ & $32^{\circ} 32^{\prime} 28^{\prime \prime}$ & 608 \\
M 51 & $13^{\mathrm{h}} 29^{\mathrm{m}} 52^{\mathrm{s}} .5$ & $47^{\circ} 11^{\prime} 46^{\prime \prime}$ & 470 \\
M 83 & $13^{\mathrm{h}} 37^{\mathrm{m}} 00^{\mathrm{s}} .7$ & $-29^{\circ} 51^{\prime} 58^{\prime \prime}$ & 510 \\
NGC 6946 & $20^{\mathrm{h}} 34^{\mathrm{m}} 51^{\mathrm{s}} .9$ & $60^{\circ} 09^{\prime} 15^{\prime \prime}$ & 46 \\
\hline
\end{tabular}

in Sect. 4, where also some conclusions following from this work are presented. The basic data obtained from this survey are collected in Table 3, which can also be found in Sect. 4.

A more detailed analysis of the physical properties in the ISM of the observed galaxies is beyond the scope of this paper. On some of the observed objects more detailed investigations will be conducted in forthcoming publications.

\section{Observations and data reduction}

We used the Heinrich-Hertz-Telescope ${ }^{1}$ (Baars \& Martin 1996; Baars et al. 1999), located on Mt. Graham, Arizona, during four periods of variable length in April 1998, December 1998, April 1999, and January 2000, to obtain the data presented in this paper.

We usually had several hours of good atmospheric conditions with a zenith opacity between 0.2 and 0.8 at $345 \mathrm{GHz}$. For the observations we used a 2-channel SIS receiver (with the channels sensitive to orthogonal polarizations), provided by the MPIfR Bonn. Each of the two receiver channels was connected to a 2048-channel AOS, providing a total bandwidth of $1 \mathrm{GHz}$ and a channel separation of $0.48 \mathrm{MHz}$ (velocity resolution $\sim 0.9 \mathrm{~km} \mathrm{~s}^{-1}$ ). System temperatures for both receiver channels were typically between 600 and $1200 \mathrm{~K}$.

All results in this paper are given in main beam brightness temperatures $\left(T_{\mathrm{mb}}\right)$, which are related to the antenna

1 The HHT is operated by the Submillimeter Telescope Observatory on behalf of Steward Observatory and the MPI für Radioastronomie. 
temperature, corrected for atmospheric absorption and rear spillover via $T_{\mathrm{mb}}=\left(F_{\text {eff }} / B_{\text {eff }}\right) T_{\mathrm{A}}^{*}$ (the IRAM calibration scheme). The main beam efficiency of the HHT at $345 \mathrm{GHz}$ is about 0.46 and the forward efficiency about 0.92 (Wilson et al. 2000).

We used the wobbler-switch mode for our observations, with the subreflector wobbling with a frequency of $2 \mathrm{~Hz}$ and the reference positions located at $\pm 4^{\prime}$. All target observations were made on a rectangular grid in RADec-orientation with a pixel separation of $10^{\prime \prime}$ in each direction, in order to achieve a full sampling for the given beamwidth of about $22^{\prime \prime}$. We regularly observed strong continuum sources (planets, W3OH) and also point-like spectral line sources to check the pointing accuracy. We also checked the focussing of the telescope two times a day on a planet. While the focus parameters were stable within $\pm 0.03 \mathrm{~mm}$ and the pointing accuracy was usually about $3^{\prime \prime}$, the observations of some days in April 1998 suffered from larger pointing errors (up to $15^{\prime \prime}$ ) due to an encoder problem. We could, however, overcome this problem by regularly mapping the inner parts of the target galaxies, where line shapes change rapidly. This allowed an accurate relative positioning of the individual observations of each galaxy. An absolute pointing accuracy of $5^{\prime \prime}$ (maximum errors) was finally obtained. This was also confirmed by re-observing every source after the encoder problem was fixed. This pointing problem has only a negligible effect on our absolute calibration (because our sources are not point-like). But together with a small misalignment of both receiver channels due to the complicated optics of the receiver it may degrade the beam width of the reduced and co-added spectra from nominal 22" (HPBW) to about $24^{\prime \prime}$.

The temperature scale calibration procedure is described by Mauersberger et al. (1999); the absolute calibration was checked by regular observations of galactic spectral line sources. We did not find any systematic deviations of our results from the brightness temperatures listed in Stanek et al. (1995). However, some additional calibration uncertainty can arise from a sideband ratio $\neq 1$ with the used DSB receiver, and from the fact that the sideband ratio may have varied for individual tunings. Hence the absolute calibration scale may be accurate to $15 \%$, what is not unusual in the sub-mm range. The relative calibration scale could be checked by re-observing the central region of each object for every individual tuning. After adjusting the temperature scale of some data sets, the remaining relative calibration uncertainty is $<3 \%$ for objects with narrow lines (like NGC 278 and IC 342) and may be up to $8 \%$ over the whole mapped region for objects which cover a large velocity range, or between various objects at different velocities. The error estimates given in Sect. 3 take into account only the relative uncertainties (besides the spectral noise), since only these are important for a comparison between the objects and various regions within one object.

The data reduction was performed using the GILDASpackage including the CLASS and GRAPHIC programs in a standard manner. Baselines of first order were subtracted from each spectrum. In many cases the baselines of receiver channel 2 were curved; from these spectra we subracted also baselines of second or third order and checked the results by comparison with the corresponding spectra of channel 1. Finally several individual spectra on each position of the observed region were coadded with an appropriate weighting.

\section{Results}

The target galaxies selected for this study and for which we have obtained extended maps are listed in Table 1. Some important data from the literature are compiled in Table 2, while our results are collected in Table 3 . These include the measured line flux (i.e. the velocity-integrated line intensity, spatially integrated over the central emission peak), the power radiated in the $\mathrm{CO}(3-2)$ line (also spatially integrated), the spatial extent of the central emission peak, the emitted power normalized to the size of the emitting region, line ratios in the centre and the disk, and the line width in the centre, which is not corrected for different inclinations. Both Tables 2 and 3 can be found in Sect. 4. In this section we will refer to line ratios $R$, total fluxes $F$, and other terms; their definitions can also be found in Sect. 4.

For each galaxy we present a set of figures illustrating the basic results. These are (a) a raster map of the spectra we obtained within a galaxy, (b) a contour map of the velocity-integrated line intensity, overlaid on an optical image extracted from the Digitized Sky Survey ${ }^{2}$, (c) a position-velocity diagram along the major axis which is defined by the position angle (ccw from north) in Table 2, and (d) a small graph ${ }^{3}$ showing the measured variation with galactocentric distance of the velocity-integrated intensity $I_{\mathrm{CO}(3-2)}$, both along the major and minor axis. While the raster maps (a) are shown with velocity resolutions of $\Delta v=10 \mathrm{kms}^{-1}$ in the central regions and $20 \mathrm{~km} \mathrm{~s}^{-1}$ in the outer parts for some galaxies, the other figures and the data analysis are done using data cubes with $\Delta v=10 \mathrm{~km} \mathrm{~s}^{-1}$ for all positions. For the positionvelocity diagrams (c) and the intensity distribution (d) the offsets along the axes are always chosen in a way that increasing offsets correspond to increasing declinations. This means that the strips go either from southeast to northwest or from southwest to northeast, even if this seems to be in contradiction with the usual definition of the position angle, which is counted up to $180^{\circ}$ counterclockwise from north. Further we note that for some of the galaxies (NGC 253, Maffei 2, IC 342, NGC 2146, M 82, and NGC 4631) the zero positions of figures (c) and (d) do not

\footnotetext{
${ }^{2}$ The DSS images are based on photographic data obtained using Oschin Schmidt Telescope on Palomar Mountain. The Digitized Sky Survey was produced at the Space Telescope Science Institute under U.S. Government grant NAG W-2166.

${ }^{3}$ We acknowledge the use of the GRACE program for plotting purposes
}

(http://plasma-gate.weizmann.ac.il/Grace/). 
coincide with the central positions given in Table 1 , but are shifted to the $\mathrm{CO}(3-2)$ maxima; see the corresponding subsections for details. This was done in cases where a wrong reference position for the cuts along the major and minor axis could have lead to incorrect results. In figure parts (a) and (b), however, the $(0,0)$ position always corresponds to that given in Table 1 .

The spectra of three of the galaxies (NGC278, NGC 4631, and M51) have already been published by Wielebinski et al. (1999); nevertheless we have included these in order to have a complete sample of galaxies which are analysed in the same manner. This provides better statistics for our conclusions.

The rms noise in our spectra depends on the observing time spent on each point. The latter was chosen according to the strength of the signal and the location of the grid point within the galaxy. For the final velocity resolution of $10 \mathrm{~km} \mathrm{~s}^{-1}$, near the centre of the mapped objects the channel-to-channel rms is ranging from $20 \mathrm{mK}$ to about $60 \mathrm{mK}$. Near map edges the rms is generally increasing, reaching about $160 \mathrm{mK}$ at worst. In general, the uncertainties due to spectral noise are smaller than the overall calibration uncertainty as discussed in Sect. 2. We further note that any absolute calibration error is of a systematic nature and has a similar effect for all objects in our sample, minimizing the effect on the conclusions one can draw from a comparison of the galaxies.

\subsection{NGC 253}

The Sculptor galaxy NGC 253 is one of the nearest $(D=$ 2.5 Mpc; Mauersberger et al. 1996 and references therein) spiral galaxies which exhibit strongly enhanced star formation (a "starburst") in its central region (Rieke et al. 1988). It is nearly edge-on $\left(i=78^{\circ}\right.$, Pence 1981) and is classified as $\mathrm{SAB}(\mathrm{s}) \mathrm{c}$. The starburst nucleus of NGC 253 is one of the brightest extragalactic sources from the infrared to cm-wavelengths. It is presumably triggered by the potential of a bar that is visible in many wavelength ranges.

Due to its small distance and its luminosity this galaxy has already been studied by several authors in detail. There are also numerous investigations of the CO gas. Wall et al. (1991) observed NGC 253 in the (2-1) and (3-2) lines of the isotopomers ${ }^{12} \mathrm{CO}$ and ${ }^{13} \mathrm{CO}$. They analysed the data to determine excitation conditions for the major and minor axis. Large maps of the $\mathrm{CO}(1-0)$ and the $\mathrm{CO}(2-1)$ emission of this galaxy were obtained by Houghton et al. (1997) and Mauersberger et al. (1996). The higher transitions (3-2) and (4-3) of the CO molecule were also observed, and maps are presented by Israel et al. (1995). The most recent CO study, including the three lowest transitions of the most abundant isotopomers, was published by Harrison et al. (1999).

Morphology. The extent of this galaxy is several arcminutes, but our observations are restricted to the inner part of the optical disk, out to a radius of about $65^{\prime \prime}(800 \mathrm{pc})$. The data we obtained are shown in Fig. 1. The highest brightness temperatures in the central region reach values of 3 to $4 \mathrm{~K}$. Israel et al. (1995) measured somewhat higher temperatures $(5-6 \mathrm{~K})$. These are not in contradiction with our values due to the smaller beam of the JCMT. However, Wall et al. (1991) also observed peak temperatures of about $6 \mathrm{~K}$ in the $\mathrm{CO}(3-2)$ line with the CSO, thus with a beam size similar to our observations.

The central position of our observations was originally chosen at the coordinates given by Young et al. (1995). It was shifted afterwards to the value given in Table 1 to better fit to the central position often given by other authors. The intensity peak of the $\mathrm{CO}(3-2)$ data, moreover, is located at the position given by Mauersberger et al. $(1996)$, which is at $(\Delta \mathrm{RA}, \Delta$ Dec. $)=\left(5^{\prime \prime} .4,6^{\prime \prime} .0\right)$ relative to the $(0,0)$ position. This was taken as reference point for the data analysis (Figs. 1c and d).

The morphology of the observed $\mathrm{CO}(3-2)$ emission is - considering the different beams - similar to the maps given by Israel et al. (1995). We cannot resolve the two intensity peaks (which are separated by $\sim 10^{\prime \prime}$ ) in the total intensity map, but a twin-peak structure is suggested by the position-velocity diagram along the major axis of NGC 253, with the stronger peak in the southwest. The source size can be estimated by Gaussian fits along the major and minor axis. When deconvolved from the telescope beam, assumed to be $24^{\prime \prime}$ (see Sect. 2), this is $35^{\prime \prime} \times 15^{\prime \prime}$, which corresponds to a linear size of $430 \mathrm{pc} \times 180 \mathrm{pc}$ at the distance of NGC 253. These values suggest that the molecular gas temperature and density fall off relatively quickly with increasing distance from the centre, even along the bar.

Intensities. The integrated $\mathrm{CO}(3-2)$ flux of the region mapped in NGC 253 is $F_{\mathrm{CO}(3-2)}=8.74 \pm 0.30 \times$ $10^{4} \mathrm{Jy} \mathrm{km} \mathrm{s}^{-1}$. The total power radiated in the $\mathrm{CO}(3-2)$ line is $6.0 \pm 0.2 \times 10^{30} \mathrm{~W}$. Since we have not mapped the whole galaxy, but only the inner $\sim 2 \mathrm{kpc}$ of the optical disk, the total values (for the whole galaxy) are probably somewhat larger. Houghton et al. (1997) have detected $\mathrm{CO}(1-0)$ emission out to a radius of $11^{\prime}(8 \mathrm{kpc})$, hence there may be also an extended $\mathrm{CO}(3-2)$ component far away from the centre.

The maximum integrated line intensity in our map (Fig. 1b) is $I_{\mathrm{CO}(3-2)}=680 \pm 60 \mathrm{~K} \mathrm{~km} \mathrm{~s}^{-1}$. This value is somewhat lower than data given by other authors, even when observed with (or convolved to) similar telescope beams. Harrison et al. (1999) give a value of $998 \mathrm{~K} \mathrm{~km} \mathrm{~s}^{-1}$, which is consistent with $990 \mathrm{~K} \mathrm{~km} \mathrm{~s}^{-1}$ given by Israel et al. (1995). Wall et al. (1991) measured even $1200 \mathrm{~K} \mathrm{~km} \mathrm{~s}^{-1}$. Hence it seems that our value for $I_{\mathrm{CO}(3-2)}$ is about $30 \%$ below the other values, and this difference is larger than our overall calibration uncertainty. It is very unlikely that we have lost some emission due to incorrect baseline subtraction. We should also note that data for this galaxy were taken during different observing periods, and the individual data sets were consistent with each other. Hence there is no obvious explanation for the intensity differences 
(a) NGC253 ${ }^{12} \mathrm{CO}(3-2)$

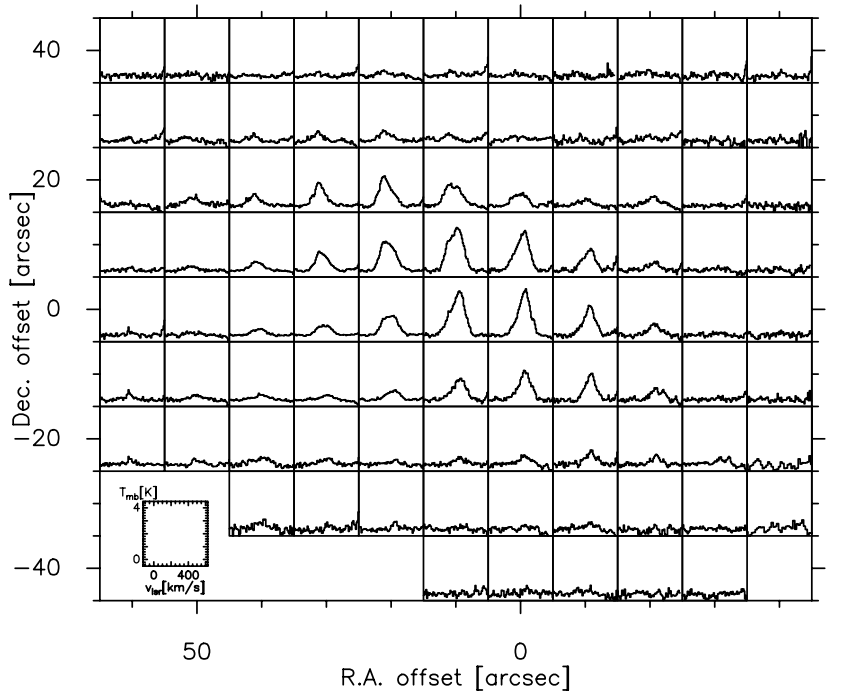

(c)

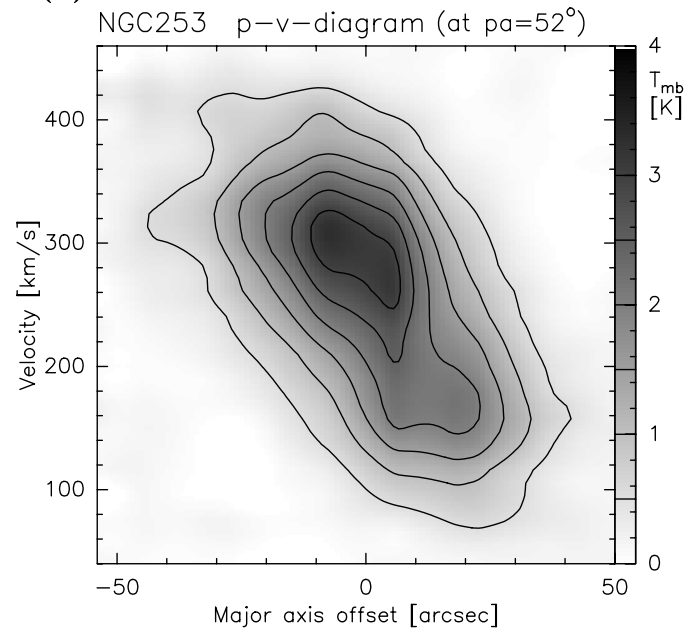

(d) (b)

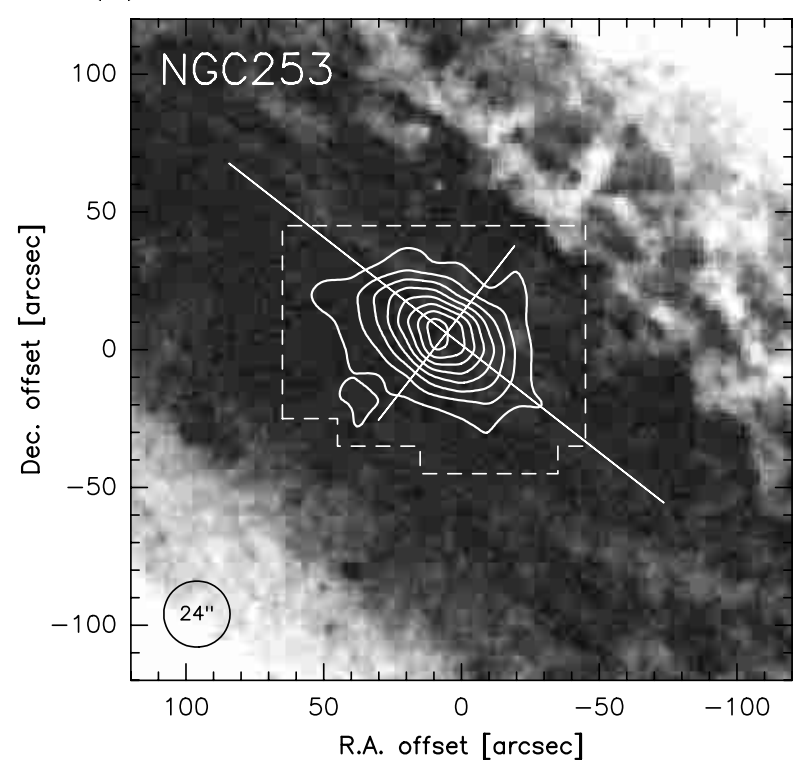

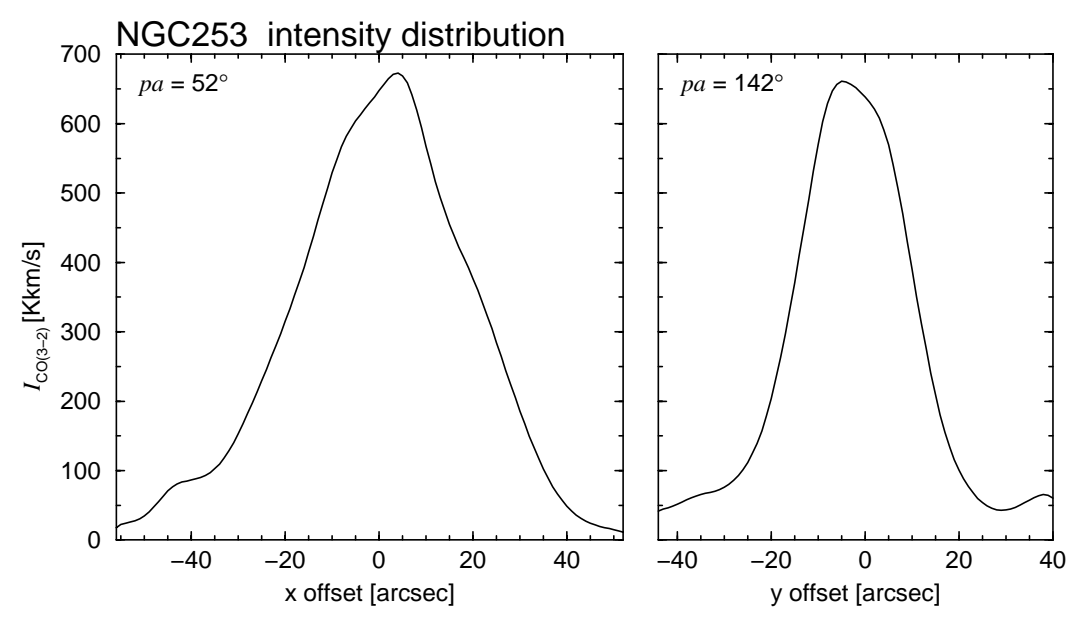

Fig. 1. CO(3-2) data of NGC 253: a) (upper left) raster map of the individual spectra, with $(0,0)$ corresponding to the position given in Table 1. The scale of the spectra is indicated by the small box inserted in the lower left corner of the image. b) (upper right) contour map of the integrated intensity, overlaid on an optical image extracted from the Digitized Sky Survey. Contour levels are $80,160, \ldots, 640 \mathrm{~K} \mathrm{~km} \mathrm{~s}^{-1}$. The region covered by the spectra shown in a) is indicated by the dashed polygon, and the cross shows the reference position for data analysis (see text) and the direction of the major and minor axis. c) (lower left) position-velocity diagram along the major axis. Contours are $0.5,1.0, \ldots 3.0 \mathrm{~K}$. d) (lower right) intensity distribution along the major and minor axis, respectively. Note that for figure parts c) and $\mathbf{d}$ ) the zero position is taken from Mauersberger et al. (1996) and differs from the position $(0,0)$ in figure parts $\mathbf{a})$ and $\mathbf{b})$ by $(\Delta \mathrm{RA}, \Delta$ Dec. $)=\left(5^{\prime \prime} .4,6^{\prime \prime} .0\right)$.

compared to earlier publications. However, atmospheric calibration errors more severe than expected cannot be completely ruled out, considering the low declination of NGC 253 at the HHT.

Line Ratios. Because of this calibration difference, our estimated line ratios, using the $\mathrm{CO}(1-0)$ and the $\mathrm{CO}(2-1)$ data from Wall et al. (1991) and Harrison et al. (1999), are systematically below those estimated by these authors. We find $R_{3,1}=0.8 \pm 0.1$ and $R_{3,2}=0.7 \pm 0.1$ in the central region. For the disk, at a distance at about $30^{\prime \prime}$ from the centre, we get a slightly lower value, $R_{3,2}=0.5 \pm 0.1$. This decrease in the line ratio can also be seen in the data of Wall et al. (1991). However, the value for the central region is surprisingly low when compared with those for other starburst galaxies in our sample (see Table 3). When we assume a $\mathrm{CO}(3-2)$ line intensity $30 \%$ higher, similar to the value of Harrison et al. (1999), we would estimate $R_{3,1} \sim 1.1$ for the central region. This is still below the average value for the other starburst galaxies. A low line ratio is even more surprising when we consider that, due to the small distance to NGC 253, we sample only the very inner star-forming centre, whereas for other galaxies a larger part of the disk is also observed on the central points due to the lower linear resolution. These line ratios indicate densities of $10^{3}-10^{3.5} \mathrm{~cm}^{-3}$ at temperatures of $20-40 \mathrm{~K}$ in the centre, but $n\left(\mathrm{H}_{2}\right)<10^{3} \mathrm{~cm}^{-3}$ in 


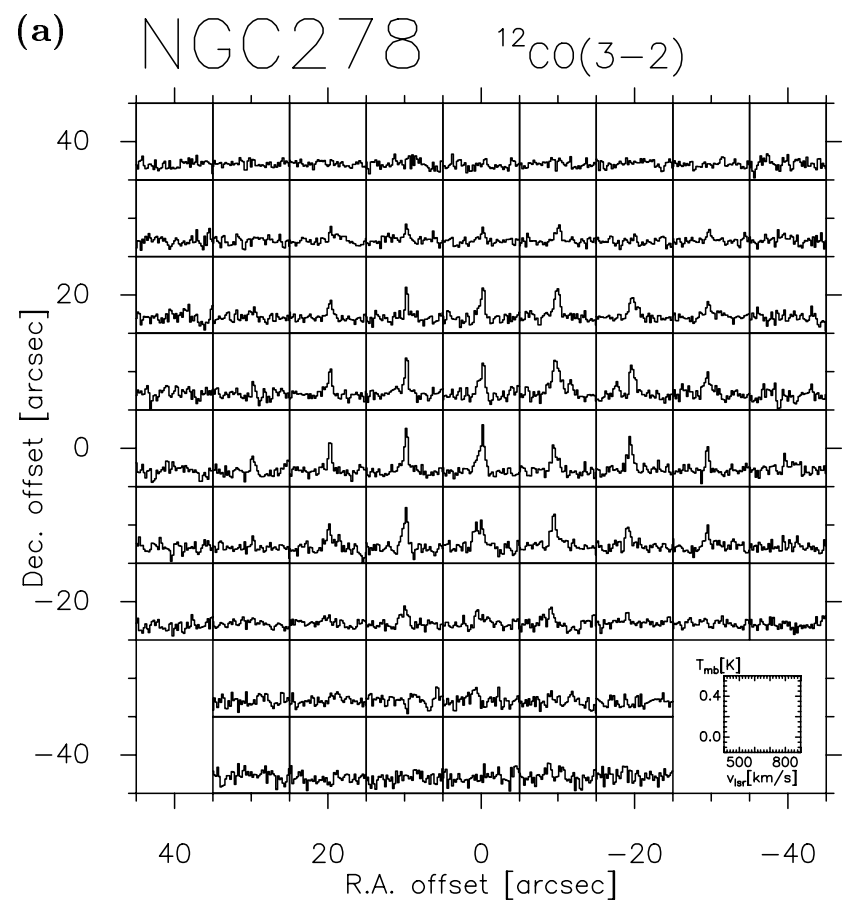

(b)

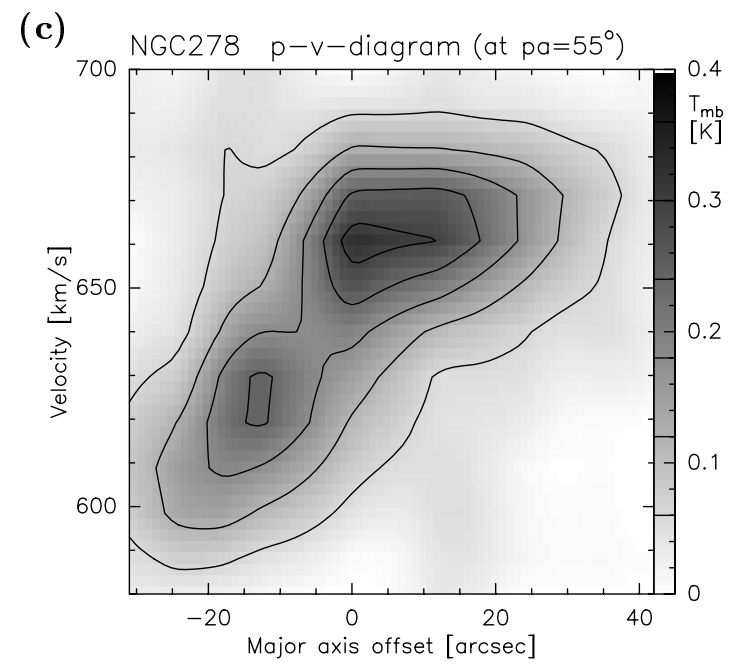

(d)
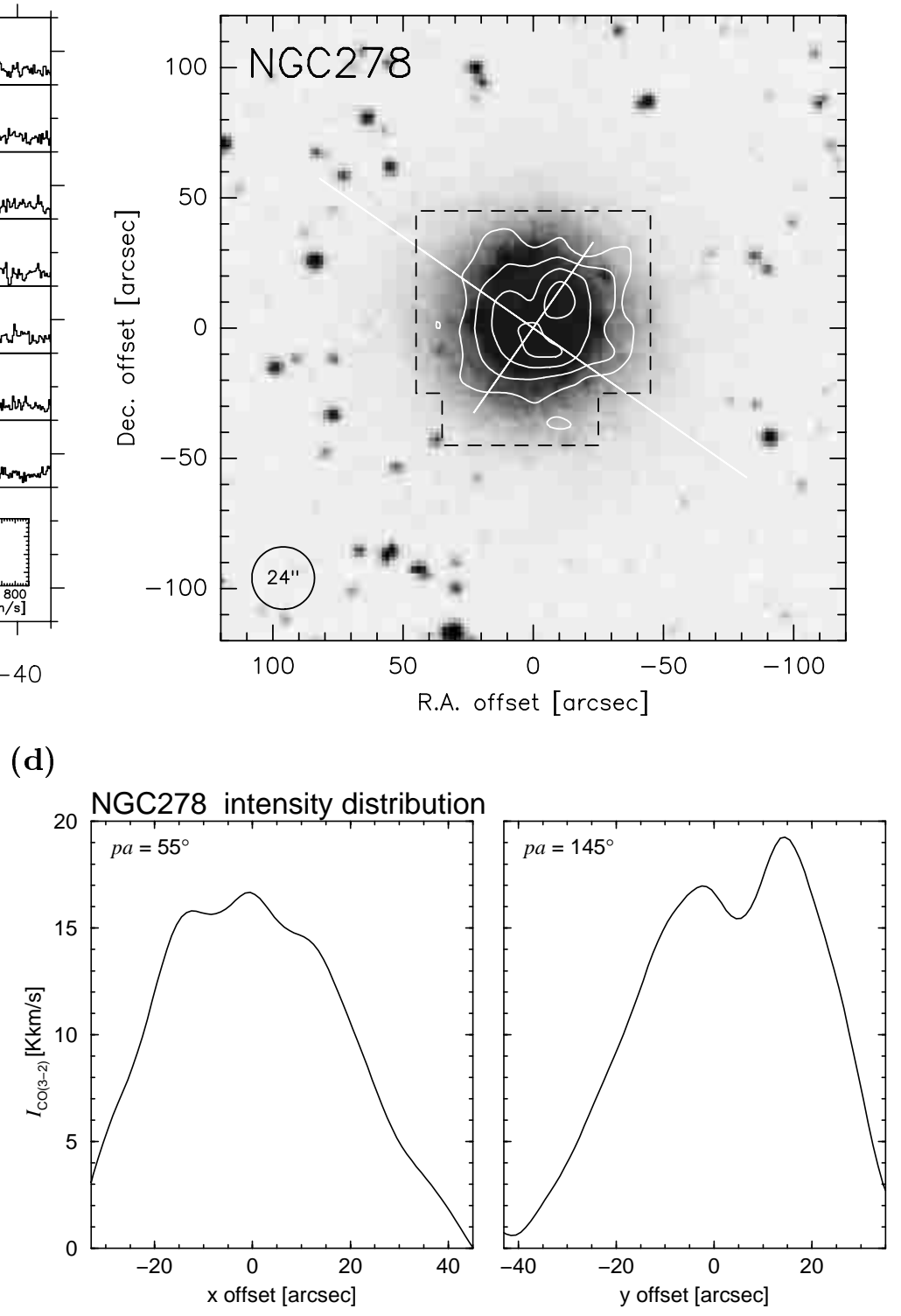

Fig. 2. CO(3-2) data of NGC 278: a) (upper left) raster map of the individual spectra, with $(0,0)$ corresponding to the position given in Table 1 . The scale of the spectra is indicated by the small box inserted in the lower right corner of the image. b) (upper right) contour map of the integrated intensity, overlaid on an optical image extracted from the Digitized Sky Survey. Contour levels are $4,8,12,16 \mathrm{~K} \mathrm{~km} \mathrm{~s}^{-1}$. The region covered by the spectra shown in a) is indicated by the dashed polygon, and the cross shows the adopted centre and the direction of the major and minor axis. c) (lower left) position-velocity diagram along the major axis. Contours are $0.06,0.12, \ldots 0.30 \mathrm{~K} . \mathbf{d}$ ) (lower right) intensity distribution along the major and minor axis, respectively.

the bar. As for all of the observed galaxies, the values given for kinetic temperature and density are only approximate, and a more detailed discussion is only possible with the use of ${ }^{13} \mathrm{CO}$ data. For NGC 253, we also refer to the publications cited above.

Kinematics. We see only the rigidly rotating part of this galaxy in the $\mathrm{CO}(3-2)$ line. The total linewidth of the $\mathrm{CO}(3-2)$ emission at the position $(10,10)$ was determined by a Gaussian fit to be $\Delta V \sim 200 \mathrm{~km} \mathrm{~s}^{-1}$. However, in the central region the velocity distribution of the emission can be described by two (or even three) components, corresponding to the double peak structure mentioned above, with a line width of about $120 \mathrm{kms}^{-1}$ for each component.

\subsection{NGC 278}

NGC 278 is a small $\left(D_{25}=2\right.$ ' 1 ), barred Sb galaxy. Its distance is $12.4 \mathrm{Mpc}$, based on the radial velocities given by Sandage \& Tammann (1981) and $H_{0}=75 \mathrm{~km} \mathrm{~s}^{-1}$. The data from this object available up to now are rather sparse. Schmidt et al. (1990) show that the nucleus of this 
galaxy is dominated by young components and suggest a recent starburst, based on emission line spectra. The CO molecule was observed in its lower rotational transitions by Braine et al. (1993) and Young et al. (1995). These authors, however, observed just a central point or three points on an arbitrarily chosen axis, respectively. In fact, the raster map shown by Wielebinski et al. (1999), which is produced from the same data set as presented in the current paper and shown in Fig. 2a, is the first mapping of the molecular ISM in this galaxy at all. Because of the sparse data, even basic morphological properties are not well known. Our data can be used to get some constraints on the orientation: in the channel maps (not shown), the largest velocity gradient can be found from southwest to northeast, along a position angle of $\sim 55^{\circ}$ (ccw from north). Hence this will be taken as position angle in the further analysis.

Morphology. The $\mathrm{CO}(3-2)$ emission is distributed throughout the entire optical disk. This is probably due to the limited spatial resolution of our data. Two emission maxima are visible in the northwest and southeast, i.e. above and below the central region along the minor axis (perpendicular to the largest velocity gradient). The interpretation depends strongly on the (unknown) inclination of the galaxy. In comparison with the NIR morphology (Rhoads 1998) these emission maxima most probably arise at both ends of a molecular bar. However, more CO data at higher angular resolution are needed for further discussion.

The intensity distribution of the $\mathrm{CO}(3-2)$ emission along the major and minor axis (Fig. 2d) is resolved and non-Gaussian. The $\mathrm{CO}(3-2)$ emission is not confined to a central region, but distributed over the whole (optically visible) galaxy. From a Gaussian fit to the intensity distribution we would determine a deconvolved source size of $38^{\prime \prime} \times 39^{\prime \prime}$. When we assume a disk-like distribution, the deconvolved source size would be rather $45^{\prime \prime} \times 46^{\prime \prime}$, which seems to be more appropriate for NGC 278. This corresponds to a linear size of $2.7 \mathrm{kpc} \times 2.8 \mathrm{kpc}$. It represents the extended $\mathrm{CO}(3-2)$ emission, not the size of a central emission peak, as for the other objects.

Intensities and Line Ratios. The integrated $\mathrm{CO}(3-$ 2) line flux of $\mathrm{NGC} 278$ is $F_{\mathrm{CO}(3-2)}=3.7 \pm 0.3 \times$ $10^{3} \mathrm{Jy} \mathrm{km} \mathrm{s}^{-1}$. The total power radiated in this line is $P_{\mathrm{CO}(3-2)}=6.2 \pm 0.5 \times 10^{30} \mathrm{~W}$. While this value is similar to that for other galaxies, in NGC 278 this power is radiated from a much larger region.

Braine et al. (1993) measured line intensities of 18 and $17 \mathrm{~K} \mathrm{~km} \mathrm{~s}^{-1}$ for the $\mathrm{CO}(1-0)$ and the $(2-1)$ line respectively. The intensity maximum in our map is $I_{\mathrm{CO}(3-2)}=$ $21 \pm 3 \mathrm{~K} \mathrm{~km} \mathrm{~s}^{-1}$. The spectra of Braine et al. (1993), however, were taken at a position which is $\left(-10^{\prime \prime}, 0^{\prime \prime}\right)$ relative to our central position which is given in Table 1 . For this position the integrated intensity is only $15 \pm 3 \mathrm{~K} \mathrm{~km} \mathrm{~s}^{-1}$. Hence we estimate line ratios of $R_{3,1}=R_{3,2}=0.8 \pm 0.2$, which were given by Wielebinski et al. (1999). These line ratios are consistent with molecular gas densities of $10^{3}-10^{3.5} \mathrm{~cm}^{-3}$ at temperatures of $20-40 \mathrm{~K}$.

The line ratios, total flux, and the extent of the emission region indicate that the amount of molecular gas is much smaller than in other galaxies, but the physical conditions in the molecular gas are not too different. This may suggest an even earlier Hubble type than Sb for this galaxy, assuming that much of the molecular gas has already been used up for star formation. A more detailed investigation of the line ratios has to await a complete mapping with higher angular resolution, including also the lower line transitions.

Kinematics. The lines in NGC 278 are relatively narrow, $\Delta V \sim 40 \mathrm{~km} \mathrm{~s}^{-1}$. This, as well as the low apparent rotational velocity of $\sim 40 \mathrm{~km} \mathrm{~s}^{-1}$, indicates that NGC 278 is oriented relatively face-on. At a distance of $12.4 \mathrm{Mpc}$, the angular diameter of $D_{25}=2$ '. 1 corresponds to about $8 \mathrm{kpc}$, which is much smaller than for normal spiral galaxies, and indicates also a lower rotational velocity. For an assumed real rotational velocity in the order of $100 \mathrm{~km} \mathrm{~s}^{-1}$ we can roughly estimate an inclination of about $20^{\circ}-25^{\circ}$.

\subsection{NGC 891}

NGC 891 is a nearby ( $D=9.6 \mathrm{Mpc}$, consistent with $H_{0}=75 \mathrm{~km} \mathrm{~s}^{-1}$; e.g. Allen et al. 1978), nearly exactly edge-on $\left(i=88^{\circ}\right)$ Sb galaxy which is often cited as being similar to our Milky Way. Due to this similarity and its orientation (which allows us to study the halo gas) this object has already been investigated at several wavelengths. Although NGC 891 has not undergone a starburst, it is an actively star-forming galaxy, which was not at least documented by the explosion of SN1986J in its southern half (e.g. Ball \& Kirk 1995). García-Burillo et al. (1992) and García-Burillo \& Guélin (1995) have mapped the CO $(2-1)$ and the $\mathrm{CO}(1-0)$ lines in this galaxy using the $30 \mathrm{~m}$ telescope on Pico Veleta. Interferometric observations were also published by Scoville et al. (1993)

Morphology. In Fig. 3 we show the first published $\mathrm{CO}(3-2)$ data of this prototype of a non-interacting, Milky Way-like spiral. The $\mathrm{CO}(3-2)$ emission seems to be relatively weak compared to the data on starburst galaxies already published. The peak temperatures do not exceed $T_{\mathrm{mb}} \sim 0.7 \mathrm{~K}$. In the central region, the (relatively narrow) main emission component is accompanied by a second, very weak and broad line, which represents the velocity components due to the central bar and which were already investigated for the lower CO transitions by García-Burillo \& Guélin (1995). While the $\mathrm{CO}(1-0)$ emission is more concentrated in the inner disk and shows a pronounced central peak, there is an interesting morphological similarity between the $\mathrm{CO}(2-1)$ and the $\mathrm{CO}(3-2)$ emission: intense, but not very extended emission regions in the centre and the southern half (at a galactocentric radius of about $50^{\prime \prime}$, and a less intense, but more extended emission region in the northern half where $\mathrm{CO}(3-2)$ emission is detectable at radii larger than $2^{\prime}$. We note that our observations did 

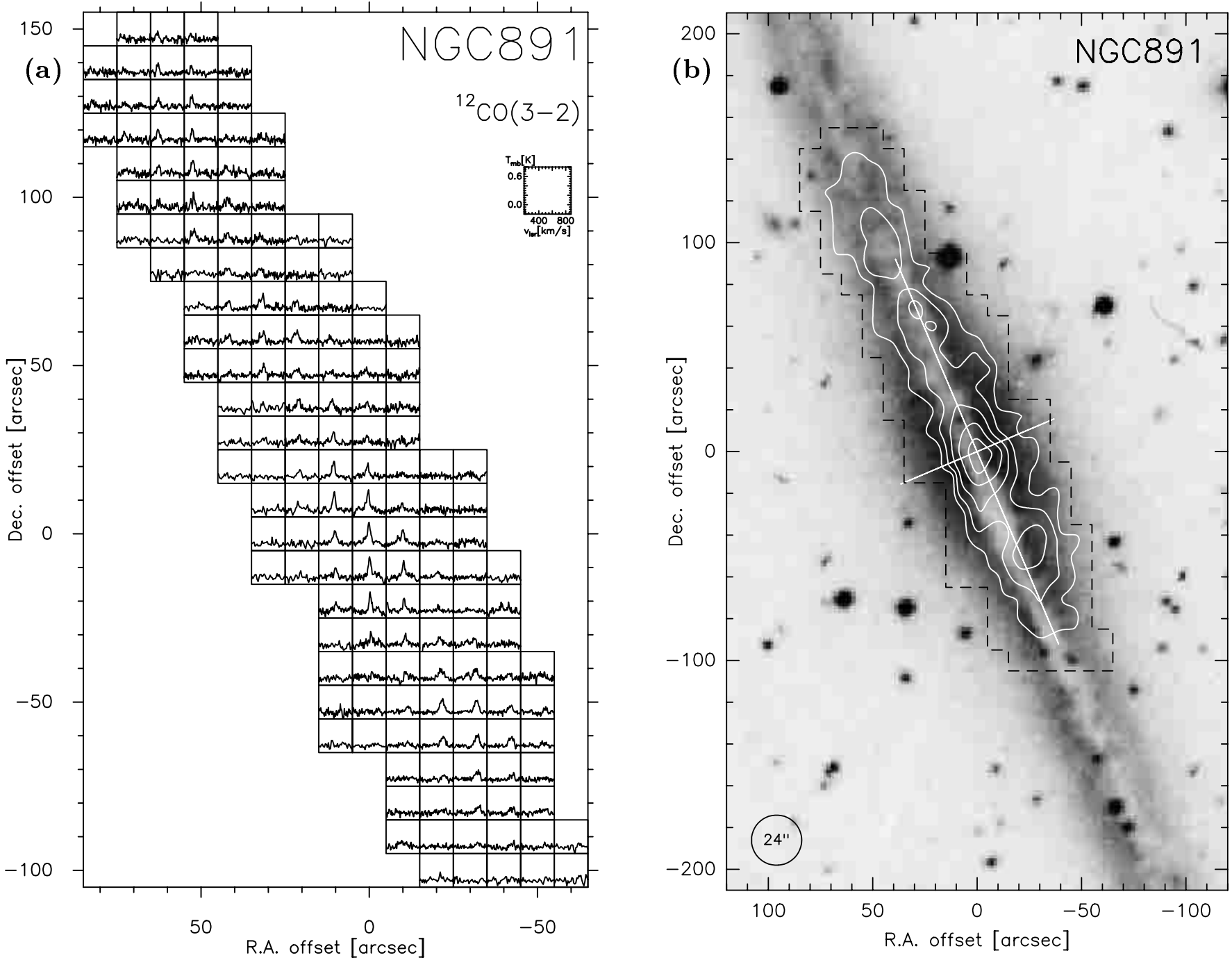

Fig. 3. a and b) $\mathrm{CO}(3-2)$ data of NGC 891: a) (left) raster map of the individual spectra, with $(0,0)$ corresponding to the position given in Table 1. The scale of the spectra is indicated by the small box inserted in the upper right corner of the image. b) (right) contour map of the integrated intensity, overlaid on an optical image extracted from the Digitized Sky Survey. Contour levels are $12,24, \ldots, 60 \mathrm{~K} \mathrm{~km} \mathrm{~s}^{-1}$. The region covered by the spectra shown in a) is indicated by the dashed polygon, and the cross shows the adopted centre and the direction of the major and minor axis.

not reach the edge of the emission at $160^{\prime \prime}$ from the centre. The shift of the southern emission peak off the major axis in western direction is visible in both the $\mathrm{CO}(2-1)$ and the $\mathrm{CO}(3-2)$ maps.

In order to estimate the size and the line flux of the central peak in NGC 891, we had to subtract the disk emission which can be found on the same line-of-sight in the fore- and background. Therefore we constructed a radial model of the off-centre emission, based on the measured intensities at $|x|>30^{\prime \prime}$, and used this model to estimate the underlying disk emission at small radii. After subtraction of this emission from the major axis intensity distribution, we find a deconvolved extent for the central $\mathrm{CO}(3-2)$ peak of $17^{\prime \prime} \times 8^{\prime \prime}$. This corresponds to $800 \mathrm{pc} \times 360 \mathrm{pc}$ at the distance of NGC 891, with the larger value along the major axis. These values concern only the central peak as noted above, emission can be detected along the whole (observed) major axis.
Intensities. Integrated over the whole region covered by our $\mathrm{CO}(3-2)$ mapping, we determine a total line flux of $F_{\mathrm{CO}(3-2)}^{\mathrm{tot}}=2.5 \pm 0.2 \times 10^{4} \mathrm{Jy} \mathrm{km} \mathrm{s}^{-1}$. This corresponds to a total power emitted in the $\mathrm{CO}(3-2)$ line of $P_{\mathrm{CO}(3-2)}^{\text {tot }}=2.5 \pm 0.2 \times 10^{31} \mathrm{~W}$ at the assumed distance of 9.6 Mpc. Since the emission does probably extend to even larger radii along the major axis, this is a lower limit. However, the amount of missed CO flux is smaller than a few percent. For a comparison of the integrated properties of this galaxy with the other objects in the sample, we estimate also the line flux of the central concentration and get, after subtraction of the disk emission in the foreand background, $F_{\mathrm{CO}(3-2)}^{\mathrm{cen}} \sim 4.7 \pm 0.6 \times 10^{3} \mathrm{Jy} \mathrm{km} \mathrm{s}^{-1}$, hence a total power radiated by the central regions of $P_{\mathrm{CO}(3-2)}^{\mathrm{cen}} \sim 4.7 \pm 0.6 \times 10^{30} \mathrm{~W}$.

Line Ratios. The $\mathrm{CO}$ data for lower transitions do not show very high brightness temperatures: these reach $T_{\mathrm{mb}} \sim 1.4 \mathrm{~K}$ for the $\mathrm{CO}(2-1)$ line and $T_{\mathrm{mb}} \sim 1.3 \mathrm{~K}$ for 

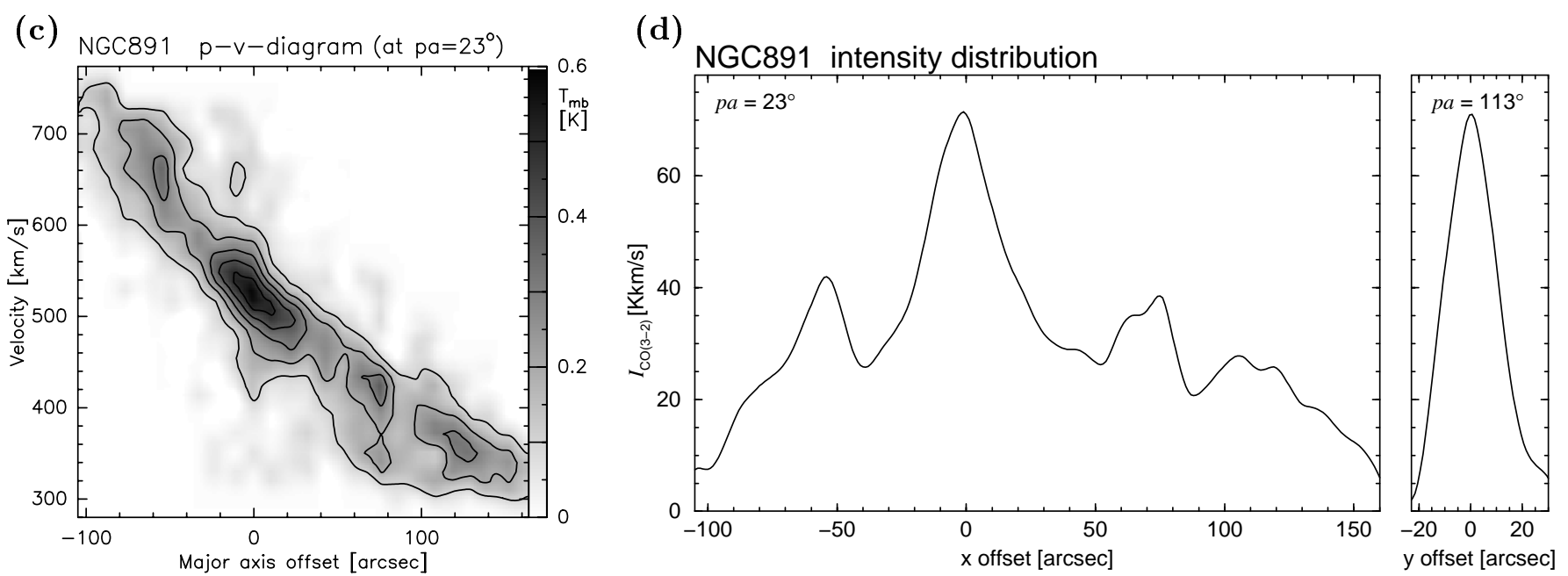

Fig. 3. c and d) $\mathrm{CO}(3-2)$ data of NGC 891: c) (left) position-velocity diagram along the major axis. Contours are 0.1, $0.2, \ldots 0.5 \mathrm{~K} . \mathbf{d})$ (right) intensity distribution along the major and minor axis, respectively.

the $\mathrm{CO}(1-0)$ line (García-Burillo \& Guélin 1995). Since the higher value for the $\mathrm{CO}(2-1)$ line is due to the smaller beam and the therefore smaller beam dilution factor, the temperatures give a line ratio of $R_{3,1} \sim 0.5$.

In the centre of NGC 891, García-Burillo et al. (1992) measured an integrated CO intensity for the (1-0) line of about $180 \mathrm{~K} \mathrm{~km} \mathrm{~s}^{-1}$ (in the $T_{\mathrm{mb}}$-scale). With a similar telescope beam size we find $I_{\mathrm{CO}(3-2)}=71 \pm 9 \mathrm{~K} \mathrm{~km} \mathrm{~s}^{-1}$, which yields a line ratio of only $R_{3,1}=0.4 \pm 0.1$. This is smaller than the value for $R_{3,1}$ we would obtain from the peak temperatures, which means that the broad lowintensity spectral component from the central bar and/or central molecular disk is weaker in the $\mathrm{CO}(3-2)$ line. This indicates lower excitation temperatures in this compenent than in the very centre. Furthermore, this line ratio is also much lower than that for the other galaxies in our sample, which typically show line ratios of $\sim 1$ or higher. In the disk, this ratio seems to increase slightly up to $R_{3,1}=$ $0.5 \pm 0.1$; the difference to the central region, however, is not significant. From these low line ratios we estimate $\mathrm{H}_{2}$ densities $<10^{3} \mathrm{~cm}^{-3}$ for the disk as well as for the central region of NGC 891.

Kinematics. The position-velocity diagram (Fig. 3c) does not show the steep velocity gradient of the central bar or molecular disk (as seen by García-Burillo et al. 1992 for the lower transitions). The kinematics of the $\mathrm{CO}(3-2)$ rather follow that of the $\mathrm{H}$ I emission (e.g. Rupen 1991). This again suggests that highly excited molecular gas, while it is ubiquitous in the spiral arms, plays only a minor role in the central molecular gas disk or in the molecular bar of NGC 891. This is different from other galaxies, where most of the $\mathrm{CO}(3-2)$ is emitted from the central region, as opposed to the spiral arms.

The line width is $\Delta V \sim 100 \mathrm{~km} \mathrm{~s}^{-1}$ at the central position, and decreases to the north and the south to about $80 \mathrm{~km} \mathrm{~s}^{-1}$. This concerns only the narrow main component. As already mentioned, the second, weaker, component due to noncircular velocities in the central region is much broader.

\subsection{Maffei 2}

Maffei 2 is a nearby starburst galaxy, classified as $\mathrm{SAB}(\mathrm{rs}) \mathrm{bc}$, and located near the galactic plane $(b=-0.5$; e.g. Spinrad et al. 1971). As distance to this object we assume the commonly used value of $D=5 \mathrm{Mpc}$ to be consistent with previous publications (e.g. Hurt et al. 1993; Hüttemeister et al. 1995). Due to its location in the sky and its systemic velocity $\left(v_{\mathrm{lsr}}=-36 \mathrm{~km} \mathrm{~s}^{-1}\right)$, molecular line observations of this object are rare. Published $\mathrm{CO}(1-$ $0)$ and (2-1) data exist from Rickard et al. (1977), Sargent et al. (1985), and Weliachew et al. (1988). The CO(3-2) line was observed by Hurt et al. (1993) with the CSO. The $\mathrm{CO}(4-3)$ line was also observed in the meantime, and will be published together with more recent $\mathrm{CO}(1-0)$ and (2-1) data (Walsh et al., in prep.).

Morphology. Our map (Fig. 4a) covers a region which is about twice as large as the region mapped by Hurt et al. (1993). Our data confirm that the $\mathrm{CO}(3-2)$ emission is as the lower transitions - concentrated to the inner disk of this galaxy. The peak brightness temperatures are between 1 and $1.5 \mathrm{~K}$, similar to the values of Hurt et al. (1993) when transformed to the $T_{\mathrm{mb}}$-scale. Their intensities, however, are slightly higher than our values. For the central position, which is also the maximum of our map, we measure a value of $I_{\mathrm{CO}(3-2)}=139 \pm 10 \mathrm{~K} \mathrm{~km} \mathrm{~s}^{-1}$. The highest value of Hurt et al. (1993) exceeds $170 \mathrm{~K} \mathrm{~km} \mathrm{~s}^{-1}$ (after smoothing to $24^{\prime \prime}$ ) and is therefore about $20 \%$ higher. These differences may have several causes - pointing or calibration uncertainties in either of both data sets. Hurt et al. (1993) do not give efficiency values, and their Fig. 1 indicates pointing difficulties. For Maffei 2, all data presented here were taken after the encoder problem mentioned in Sect. 2 has been corrected, hence the pointing accuracy for the Maffei2 data set was $3^{\prime \prime}$. 
(a) Maffei $2{ }^{12} \mathrm{co}(3-2)$

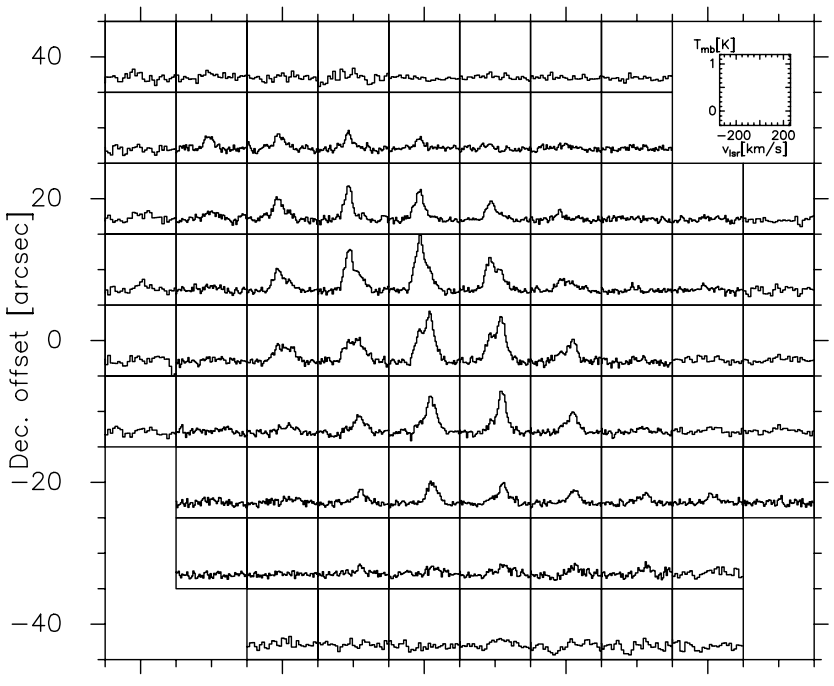

40

(c)

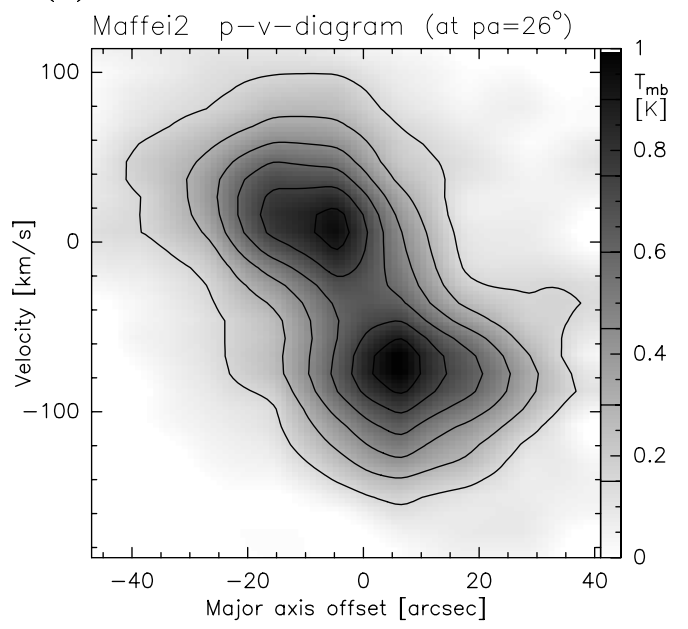

(d) (b)
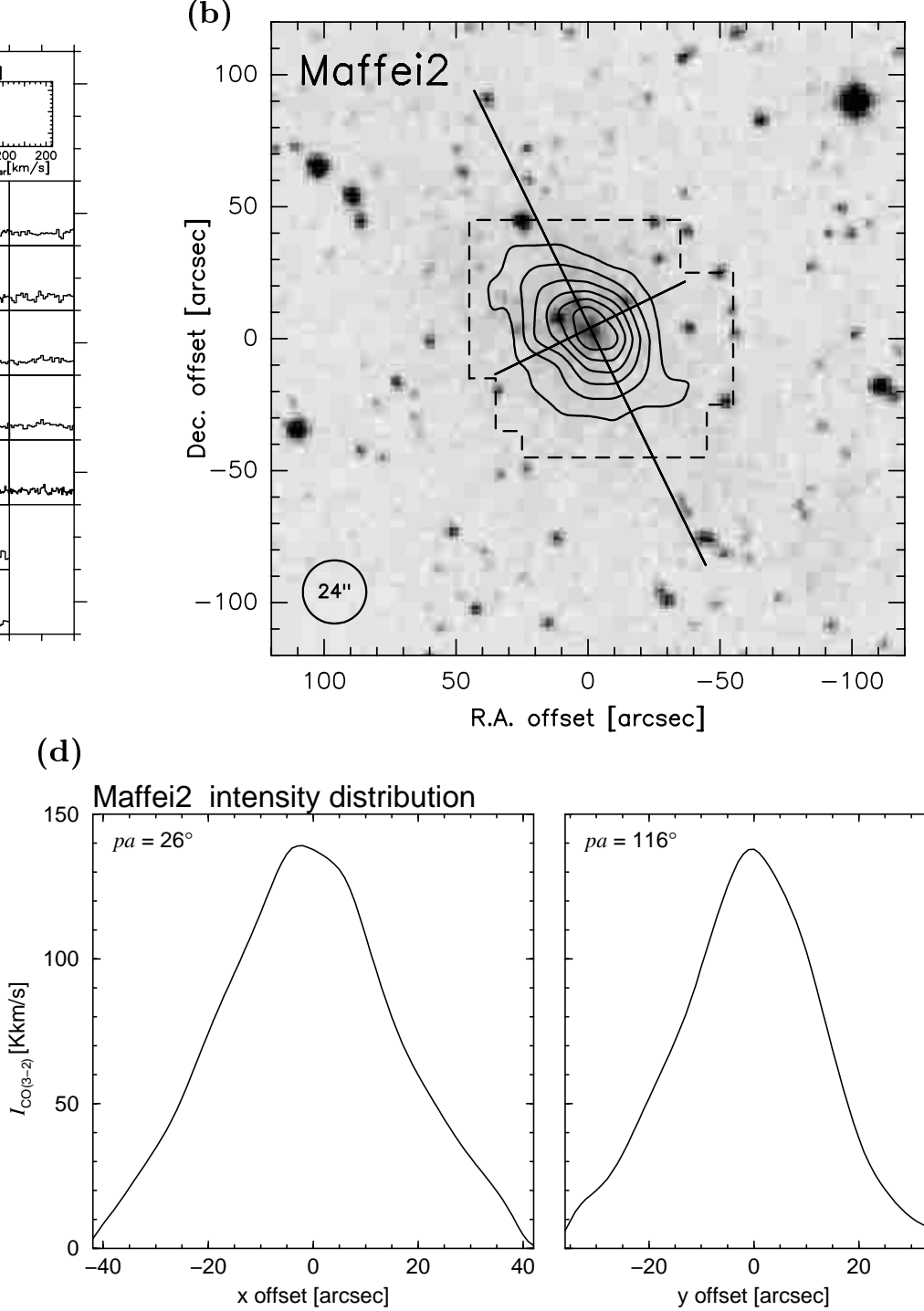

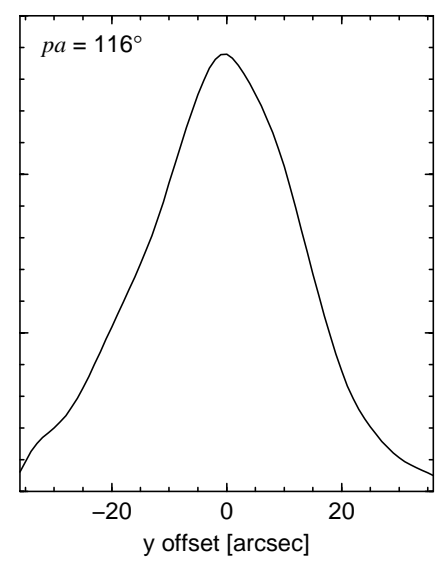

Fig. 4. $\mathrm{CO}(3-2)$ data of Maffei 2: a) (upper left) raster map of the individual spectra, with $(0,0)$ corresponding to the position given in Table 1 . The scale of the spectra is indicated by the small box inserted in the upper right corner of the image. b) (upper right) contour map of the integrated intensity, overlaid on an optical image extracted from the Digitized Sky Survey. Contour levels are $20,40, \ldots, 120 \mathrm{~K} \mathrm{~km} \mathrm{~s}^{-1}$. The region covered by the spectra shown in a) is indicated by the dashed polygon, and the cross shows the reference position for data analysis (see text) and the direction of the major and minor axis. c) (lower left) position-velocity diagram along the major axis. Contours are $0.15,0.3, \ldots 0.9 \mathrm{~K}$. d) (lower right) intensity distribution along the major and minor axis, respectively. Note that for figure parts $\mathbf{c})$ and $\mathbf{d})$ the zero position differs from the position $(0,0)$ in figure parts $\mathbf{a})$ and $\mathbf{b})$ by $(\Delta \mathrm{RA}, \Delta$ Dec. $)=\left(-0^{\prime \prime} .8,4^{\prime \prime} .0\right)$.

The reference position of our observations which is listed in Table 1 was chosen at the coordinates of Weliachew et al. (1988). For the data analysis (Figs. $4 \mathrm{c}$ and $\mathrm{d}$ ) we shifted the reference point slightly in northeastern direction onto the $\mathrm{CO}(3-2)$ maximum, located at $(\Delta \mathrm{RA}, \Delta$ Dec. $)=\left(-0^{\prime \prime} .8,4^{\prime \prime} .0\right)$ relative to the $(0$, $0)$ position. This position is identical with the reference position used by Hurt et al. (1993).

From Gaussian fits we estimate a deconvolved source size of $32^{\prime \prime} \times 21^{\prime \prime}$, corresponding to a linear size of $780 \mathrm{pc} \times$ $520 \mathrm{pc}$ at the distance of Maffei 2. Even if the intensity distribution (Fig. 4b) is centrally peaked, the shape of the individual spectra as well as the position-velocity diagram along the major axis suggest a ring-like distribution of the highly excited molecular gas, with a diameter of $10^{\prime \prime}-20^{\prime \prime}$. This is too small to be resolved by our telescope beam. A two-peaked appearance of the position-velocity diagram along the minor axis (not shown) suggests a more complicated kinematical structure, perhaps a bar which is inclined with respect to the main disk. However, the bar-like structure as seen in $\mathrm{CO}(1-0)$ interferometric maps (Hurt \& Turner 1991) is not present in our data.

Intensities and Line Ratios. The integrated line flux of Maffei 2 in the $\mathrm{CO}(3-2)$ line is $F_{\mathrm{CO}(3-2)}=1.87 \pm$ $0.08 \times 10^{4} \mathrm{Jy} \mathrm{km} \mathrm{s}^{-1}$, which corresponds at the distance to Maffei 2 to a total power of $P=5.1 \pm 0.3 \times 10^{30} \mathrm{~W}$. 
This value for $P_{\mathrm{CO}(3-2)}$ is similar to most other galaxies in our sample.

From the $\mathrm{CO}(1-0)$ data of Weliachew et al. (1988) and our observations we have determined a line ratio of $R_{3,1}=$ $1.3 \pm 0.2$ in the centre of Maffei 2 and $R_{3,1}=0.8 \pm 0.2$ in the disk (at radii of $\sim 30^{\prime \prime}$ ). This suggests high excitation temperatures in the starburst region. From a simple LVG analysis we derive densities of $n\left(\mathrm{H}_{2}\right) \sim 10^{3.5} \mathrm{~cm}^{-3}$ in the centre, falling of rapidly to $n\left(\mathrm{H}_{2}\right)<10^{3} \mathrm{~cm}^{-3}$. The kinetic temperatures in the centre must be $T>50 \mathrm{~K}$. Interestingly the estimated line ratios for Maffei 2 are higher than those for other galaxies with a ring-like molecular gas distribution, and resemble those for objects showing a more centrally peaked distribution.

Kinematics. The spectra from the central region show linewidths of $\Delta V \sim 150 \mathrm{~km} \mathrm{~s}^{-1}$. This can be attributed to two individual components, with line parameters of $V_{0} \sim$ $-85 \mathrm{~km} \mathrm{~s}^{-1}$ and $\Delta V \sim 70 \mathrm{~km} \mathrm{~s}^{-1}$ for the northeastern and $V_{0} \sim 10 \mathrm{~km} \mathrm{~s}^{-1}$ and $\Delta V \sim 90 \mathrm{~km} \mathrm{~s}^{-1}$ for the southwestern component.

\subsection{IC 342}

The SBcd galaxy IC 342 is one of the nearest grand-design spiral galaxies. Distance estimates range from $1.8 \mathrm{Mpc}$ (McCall 1989) - this value implies that infrared and CO luminosities are similar to those of the Milky Way - to $8 \mathrm{Mpc}$ (Sandage \& Tammann 1974). In order to be consistent with previous molecular line studies, we assume a distance of $D \sim 4.5 \mathrm{Mpc}$ (Xie et al. 1994; Steppe et al. 1990, and references therein).

IC 342 is oriented almost face-on $\left(i=25^{\circ}\right)$ and exhibits a nuclear starburst. Young \& Scoville (1982) observed the $\mathrm{CO}(1-0)$ transition along two perpendicular strips in this galaxy. More recent interferometric observations were performed by Ishizuki et al. (1990). Xie et al. (1994) made a study of the two lowest rotational transitions of ${ }^{12} \mathrm{CO}$ and ${ }^{13} \mathrm{CO}$ in NGC 2146 and IC 342. Also the $\mathrm{CO}(3-2)$ line (Steppe et al. 1990), and even $\mathrm{CO}(4-3)$ emission (Güsten et al. 1993), was already observed. Recently, Schulz et al. (2001) observed ${ }^{13} \mathrm{CO}$ in the $(2-1)$ and $(3-2)$ transitions using the IRAM 30-m telescope.

Morphology. We mapped IC 342 out to a galactocentric distance of about $1.3 \mathrm{kpc}$. Within this mapped region, our data show a concentration of the $\mathrm{CO}(3-2)$ emission to the inner disk of IC 342. The emitting region is slightly elongated along the major axis of the galaxy, with a deconvolved source size of $38^{\prime \prime} \times 24^{\prime \prime}$, corresponding to $840 \mathrm{pc} \times 520 \mathrm{pc}$ at the assumed distance of $4.5 \mathrm{Mpc}$. In CO data with higher spatial resolution (e.g. Schulz et al., in prep.), this elongated structure consists of at least two emission maxima, separated by $\sim 8^{\prime \prime}$. The southwest of these maxima corresponds to our original reference position (see Table 1). For the data analysis (Figs. 5c and d) we shifted the reference point by $4^{\prime \prime}$ along the major axis in northeastern direction, between these two maxima. This position, at $(\Delta \mathrm{RA}, \Delta$ Dec. $)=\left(1^{\prime \prime} .7,3^{\prime \prime} \cdot 6\right)$, corresponds to the $\mathrm{CO}(3-2)$ maximum when observed with the angular resolution of the HHT.

Intensities and Line Ratios. We find a peak intensity of the $\mathrm{CO}(3-2)$ line of $I_{\mathrm{CO}(3-2)}=148 \pm 6 \mathrm{~K} \mathrm{~km} \mathrm{~s}^{-1}$. Steppe et al. (1990) obtained higher values (with their smaller beam) especially in the northeastern part of our map. This difference may reflect the beam dilution resulting from the barred distribution of the molecular gas, as seen in the CO(1-0) line by Ishizuki et al. (1990). The CO(2-1) observations of Xie et al. (1994) which are obtained with a similar beam size as our $\mathrm{CO}(3-2)$ data allow us to directly estimate line ratios. We find $R_{3,2}=1.2 \pm 0.1$ in the centre, but values slightly smaller than 1 at $30^{\prime \prime}$ distance from the centre along the major axis. For the $(2-1) /(1-$ $0)$ ratio Xie et al. (1994) give $R_{2,1}=1.1 \pm 0.3$ for data smoothed to $45^{\prime \prime} \mathrm{HPBW}$, which corresponds to $R_{3,1} \sim 1.3$ and $\sim 1$ in the centre and the disk respectively. These values suggest a kinetic temperature of $T>50 \mathrm{~K}$ and gas densities of $n\left(\mathrm{H}_{2}\right) \sim 10^{3.5} \mathrm{~cm}^{-3}$ in the centre, but lower values in the disk. Thus the physical conditions in the molecular gas are comparable to other objects such as Maffei 2. We find an integrated line flux of $F_{\mathrm{CO}(3-2)}=$ $2.19 \pm 0.06 \times 10^{4} \mathrm{Jy} \mathrm{km} \mathrm{s}^{-1}$. With the assumed distance of $4.5 \mathrm{Mpc}$ to IC 342 , this translates to a total power emitted in the $\mathrm{CO}(3-2)$ line of $P_{\mathrm{CO}(3-2)}=4.9 \pm 0.2 \times 10^{30} \mathrm{~W}$. This value is similar to those for other spiral galaxies in our sample, but note the distance uncertainties mentioned at the beginning of this subsection (the distance enters as $D^{2}$ into the total power). The ratio $P_{\mathrm{CO}(3-2)} /\left(l_{x} \times l_{y}\right)$, i.e. the total power divided by the size of the emitting region, is $11.2 \times 10^{30} \mathrm{~W} \mathrm{kpc}^{-2}$, which is also of the same order as for some other objects in our sample.

Kinematics. A velocity gradient, consistent with normal galactic rotation, is visible along the major axis (Fig. 5c). Due to the small inclination $\left(i \sim 25^{\circ}\right)$ the line width is small, especially when compared to the edge-on objects in our sample. We determined it by a Gaussian fit to $\Delta V \sim 60 \mathrm{~km} \mathrm{~s}^{-1}$. It is relatively constant over the region where we detected $\mathrm{CO}(3-2)$ emission. Since IC 342 is classified as a barred spiral, this small line width indicates either a very particular orientation of the bar, or the absence of highly excited $\mathrm{CO}$ in the bar, since otherwise the various possible orbits would lead to a higher velocity dispersion near the centre of this galaxy.

\subsection{NGC 2146}

NGC 2146 is the most distant object in our sample. Distances cited in the literature range from 14 to $21 \mathrm{Mpc}$; we adopt $17 \mathrm{Mpc}$ throughout this study. This galaxy is classified as $\mathrm{SB}(\mathrm{s})$ ab pec, and with an inclination of $65^{\circ}$ it still can be considered as oriented edge-on. NGC 2146 is a starburst galaxy, showing a superwind driven by violent star formation in its central region (Armus et al. 1995), but has no obvious companion - a fact that distinguishes NGC 2146 from other known nearby starburst galaxies. Despite its remarkable appearance in the optical, the molecular line data on this galaxy are sparse. 


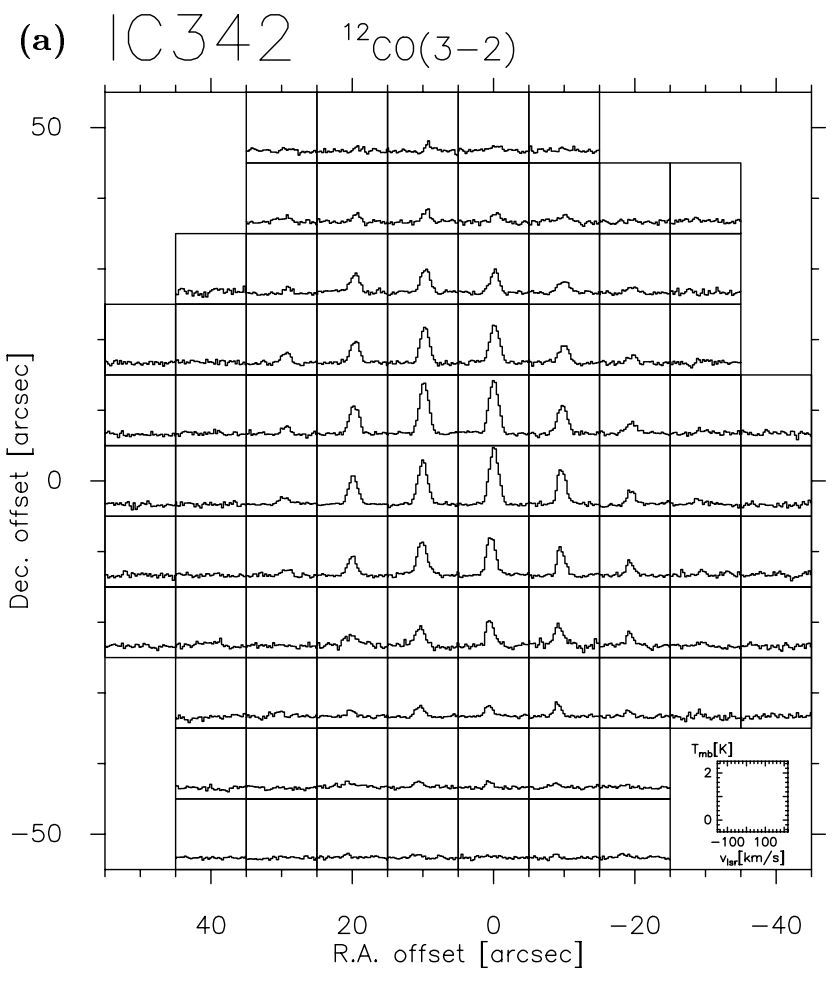

(b)

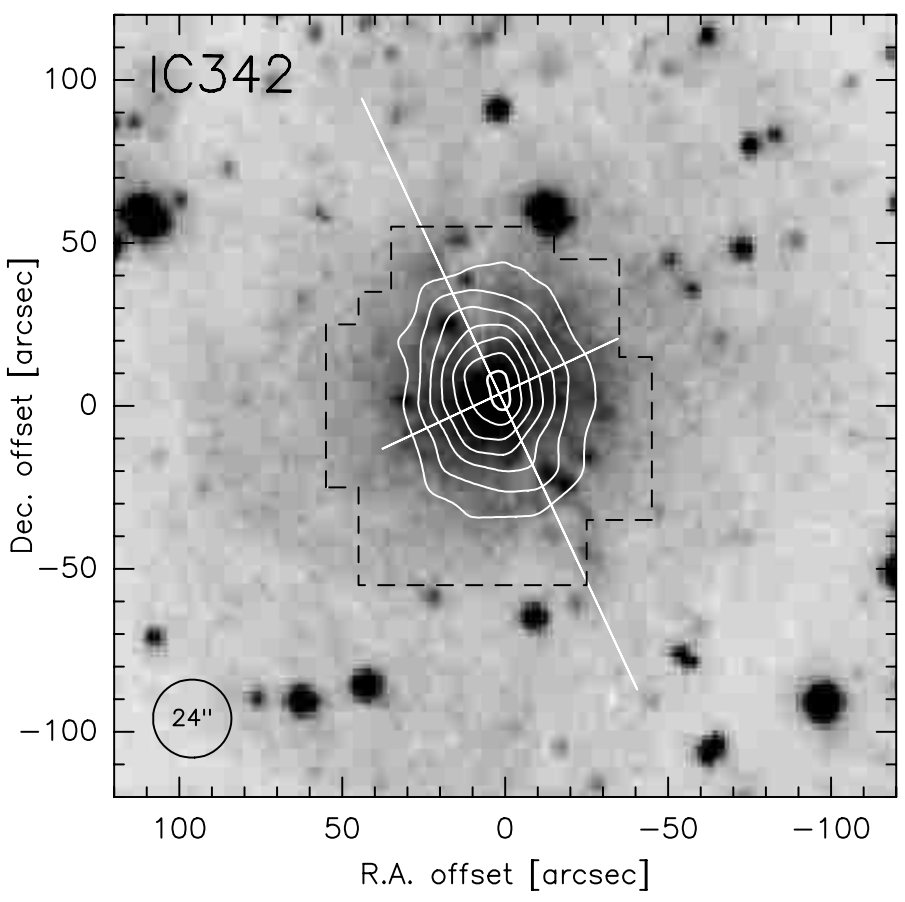

(d)
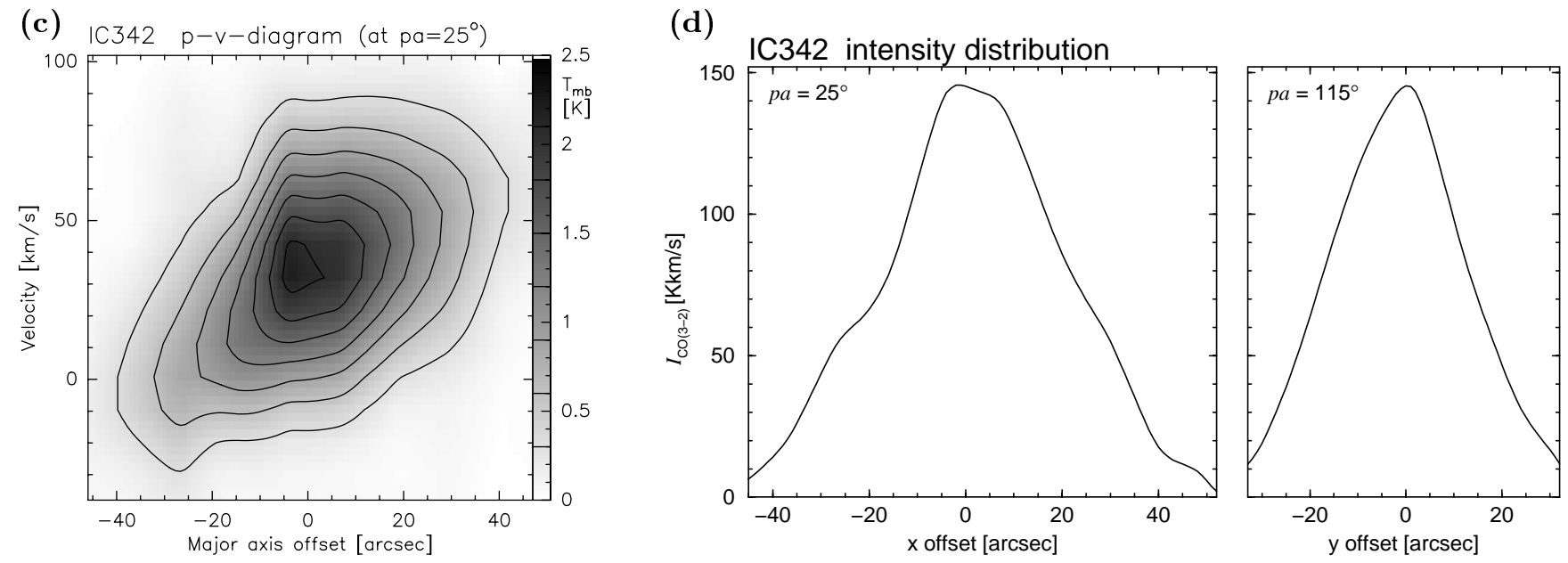

Fig. 5. CO(3-2) data of IC 342: a) (upper left) raster map of the individual spectra, with $(0,0)$ corresponding to the position given in Table 1. The scale of the spectra is indicated by the small box inserted in the lower right corner of the image. b) (upper right) contour map of the integrated intensity, overlaid on an optical image extracted from the Digitized Sky Survey. Contour levels are $20,40,60, \ldots, 140 \mathrm{~K} \mathrm{~km} \mathrm{~s}^{-1}$. The region covered by the spectra shown in a) is indicated by the dashed polygon, and the cross shows the reference position for data analysis (see text) and the direction of the major and minor axis. c) (lower left) position-velocity diagram along the major axis. Contours are $0.3,0.6, \ldots 2.1 \mathrm{~K}$. d) (lower right) intensity distribution along the major and minor axis, respectively. Note that for figure parts c) and $\mathbf{d}$ ) the zero position differs from the position $(0,0)$ in figure parts a) and b) by $(\Delta \mathrm{RA}, \Delta$ Dec. $)=\left(1^{\prime \prime} .7,3^{\prime \prime} .6\right)$.

Xie et al. (1994) observed the two lowest rotational transitions of ${ }^{12} \mathrm{CO}$ and ${ }^{13} \mathrm{CO}$, but only along the major and minor axis. A few years earlier, Jackson \& Ho (1988) obtained an interferometer map of the (1-0) transition. Recently further $\mathrm{CO}(1-0)$ and $(2-1)$ maps, covering the whole optical disk, were obtained at the IRAM 30-m telescope (Dumke et al., in prep.). There are also interferometric data for the central region made with the Plateau de Bure interferometer (Greve et al. 2000; Greve et al. in prep.). Here we present the first $\mathrm{CO}(3-2)$ data of this galaxy.

Morphology and Intensities. Because of some pointing problems during the first observing run in April 1998 (see Sect. 2) some spectra in the northern half of the mapped region had to be discarded during the data reduction, but were re-observed. The similarity between the line shapes of 
(a) NGC2146 ${ }^{12} \mathrm{Co}(3-2)$

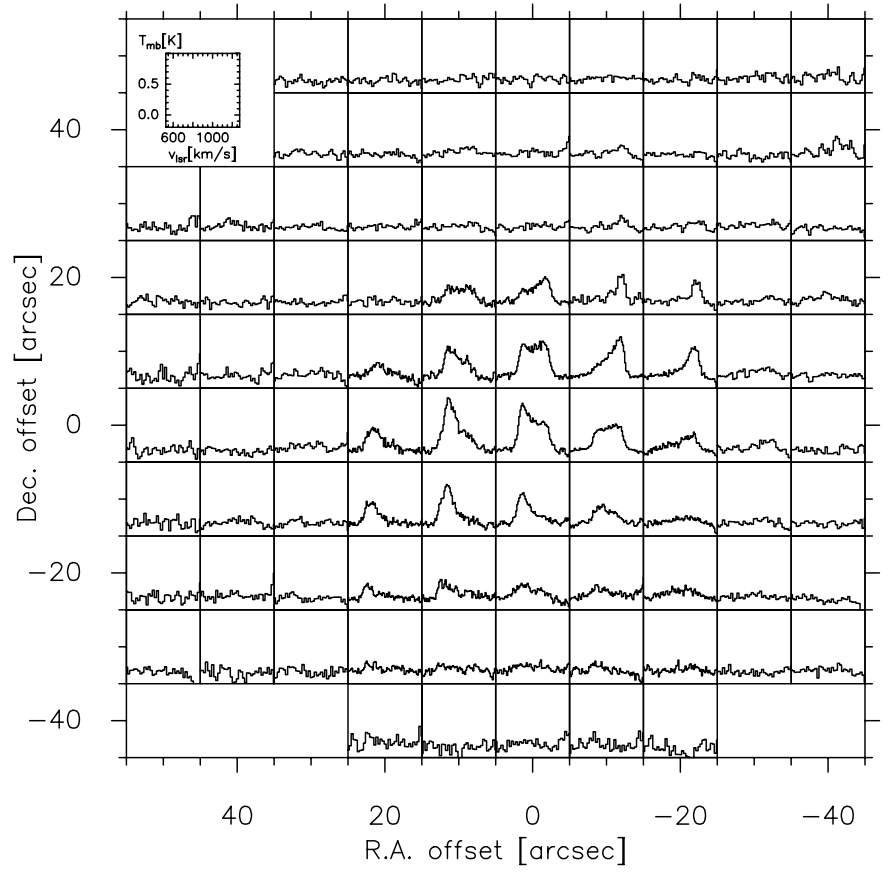

(b)

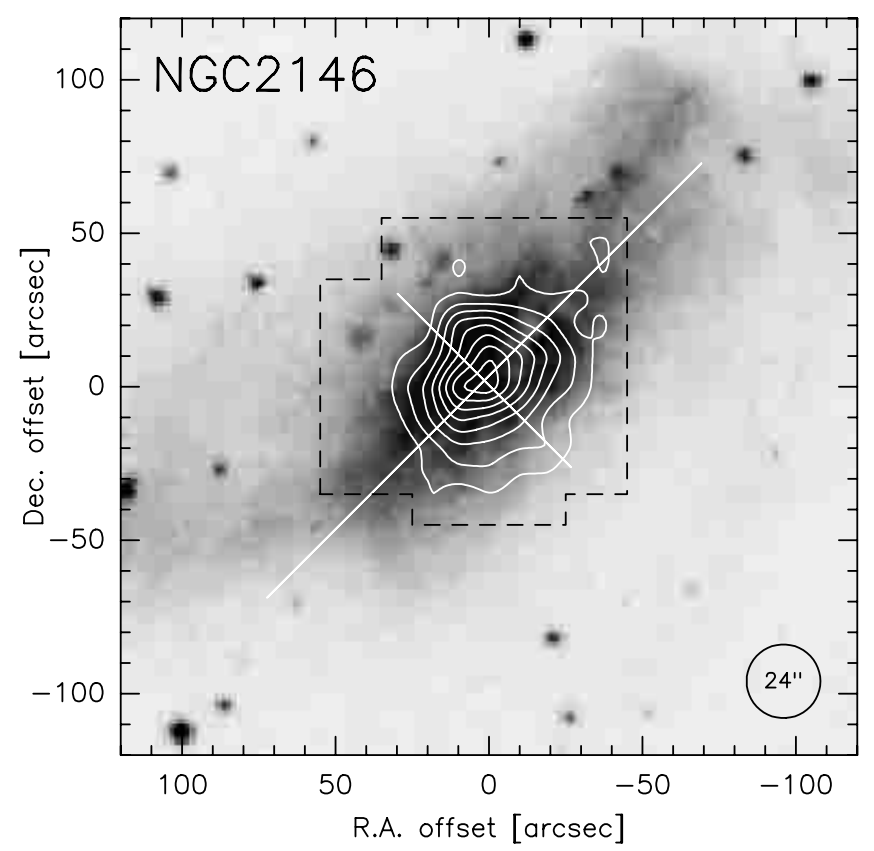

(c) $\mathrm{NGC2146} p-v$-diagram (at $\mathrm{pa}=135^{\circ}$ )

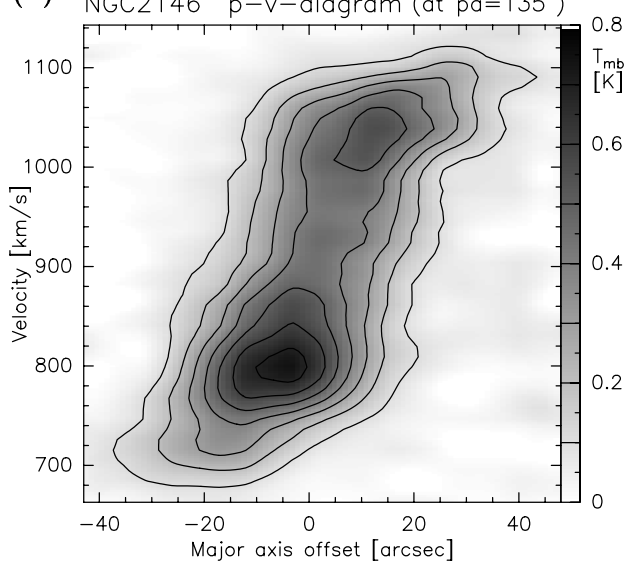

(d) NGC2146 intensity distribution

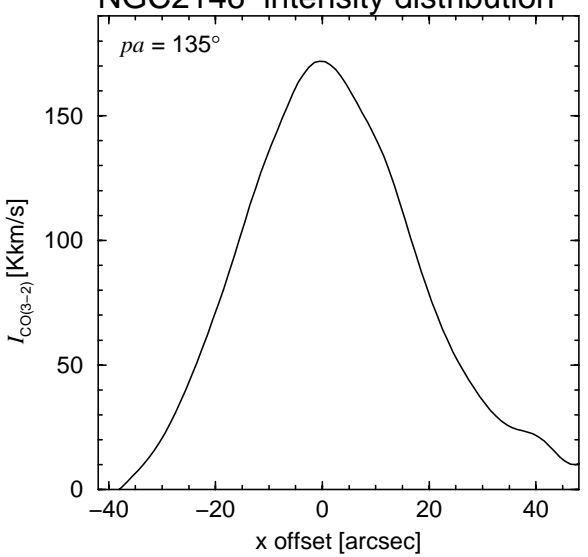

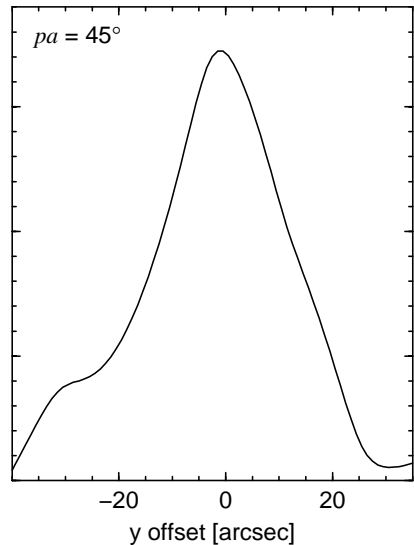

Fig. 6. CO(3-2) data of NGC 2146: a) (upper left) raster map of the individual spectra, with $(0,0)$ corresponding to the position given in Table 1. The scale of the spectra is indicated by the small box inserted in the upper left corner of the image. b) (upper right) contour map of the integrated intensity, overlaid on an optical image extracted from the Digitized Sky Survey. Contour levels are $20,40,60, \ldots, 160 \mathrm{~K} \mathrm{~km} \mathrm{~s}^{-1}$. The region covered by the spectra shown in a) is indicated by the dashed polygon, and the cross shows the reference position for data analysis (see text) and the direction of the major and minor axis. c) (lower left) position-velocity diagram along the major axis. Contours are $0.1,0.2, \ldots 0.7 \mathrm{~K}$. d) (lower right) intensity distribution along the major and minor axis, respectively. Note that for figure parts $\mathbf{c})$ and $\mathbf{d}$ ) the zero position differs from the position $(0,0)$ in figure parts a) and b) by $(\Delta \mathrm{RA}, \Delta$ Dec. $)=\left(1^{\prime \prime} .5,2^{\prime \prime} .0\right)$.

our final $\mathrm{CO}(3-2)$ map and the $\mathrm{CO}(2-1)$ data of Xie et al. (1994) shows that the pointing accuracy finally reached is relatively good.

The reference position for our observations (Table 1) was that given by Young et al. (1995). For the data analysis (see Figs. 6c and d) we shifted the reference point by $(\Delta \mathrm{RA}, \Delta$ Dec. $)=\left(1^{\prime \prime} .5,2^{\prime \prime} .0\right)$ onto the measured $\mathrm{CO}(3-2)$ maximum, and the position angle was assumed to be $135^{\circ}$. While the optical position angle is $143^{\circ}$, the major axis of the $\mathrm{CO}(1-0)$ distribution as found by interferometric observations (Jackson \& Ho 1988) is closer to $135^{\circ}$, and Xie et al. (1994) used even $128^{\circ}$ for their observations.

The CO(3-2) emission of NGC 2146 is elongated along the major axis of the galaxy, with a deconvolved source size of $29^{\prime \prime} \times 17^{\prime \prime}$. This corresponds to $2.4 \mathrm{kpc} \times 1.4 \mathrm{kpc}$ at a distance of $17 \mathrm{Mpc}$. The source extent along the minor axis is rather large. However, if we take the orientation $\left(i=65^{\circ}\right)$ and the peculiar optical appearance of this galaxy into account, it seems unlikely that the emission should be confined to a line along the position angle. The integrated line flux is $1.94 \pm 0.14 \times 10^{4} \mathrm{Jy} \mathrm{kms}^{-1}$, 

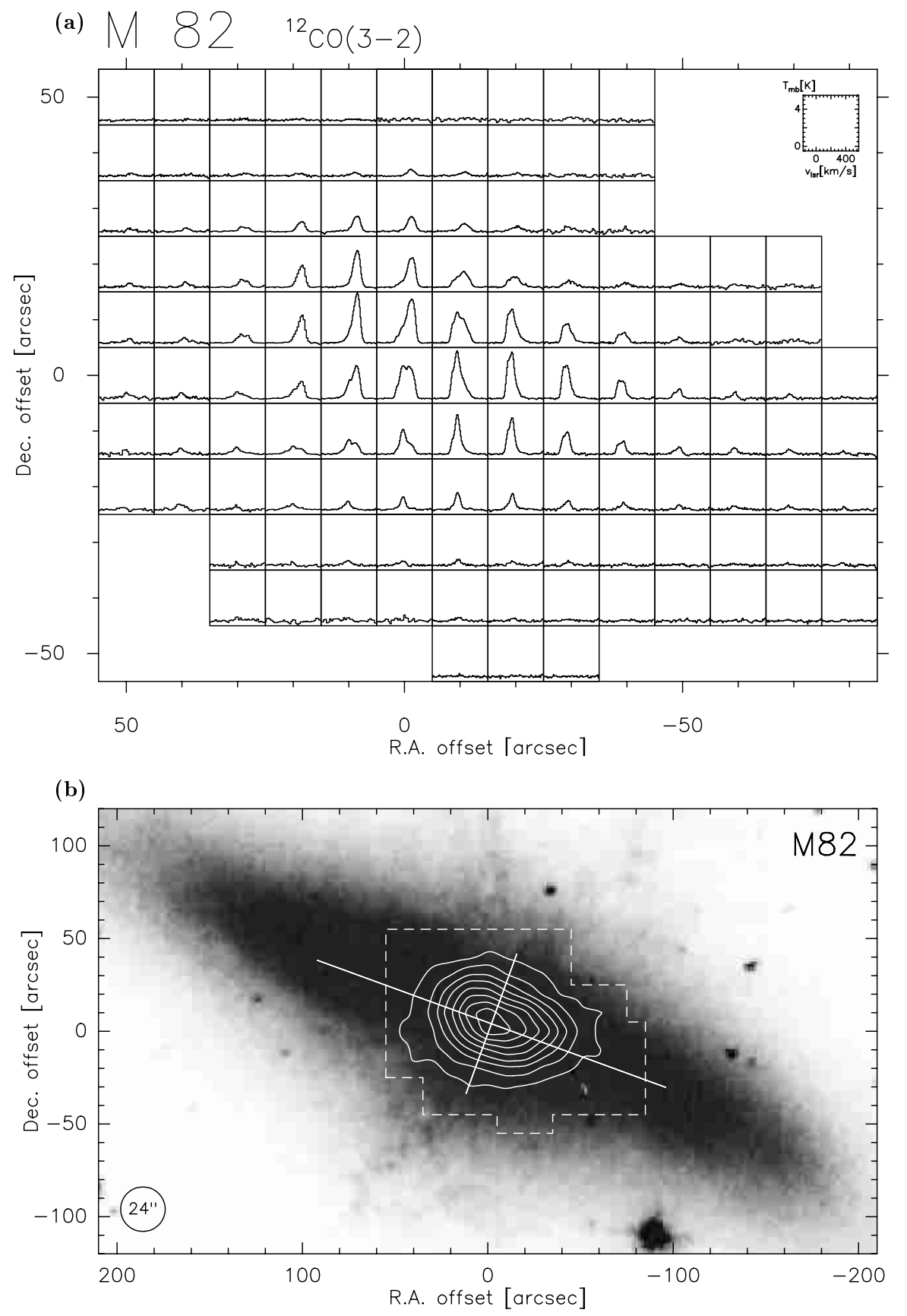

Fig. 7. a and b) $\mathrm{CO}(3-2)$ data of M 82: a) (upper) raster map of the individual spectra, with $(0,0)$ corresponding to the position given in Table 1. The scale of the spectra is indicated by the small box inserted in the upper right corner of the image. b) (lower) contour map of the integrated intensity, overlaid on an optical image extracted from the Digitized Sky Survey. Contour levels are 70, 140, 220, $300, \ldots, 700 \mathrm{~K} \mathrm{~km} \mathrm{~s}^{-1}$. The region covered by the spectra shown in a) is indicated by the dashed polygon, and the cross shows the reference position for data analysis and the direction of the major and minor axis.

but the large distance to NGC 2146 makes this the object with the largest power emitted in the $\mathrm{CO}(3-2)$ line. With $P_{\mathrm{CO}(3-2)}=6.1 \pm 0.5 \times 10^{31} \mathrm{~W}$ this galaxy is about four times as luminous as the prototype starburst galaxy M 82. This points to a strongly enhanced starburst activity in this object. This activity may probably be caused by a merging event, which can account for the disturbed appearance. It may also be the cause for physical conditions in the molecular gas that allow emission from the $J=3$ level of CO over a larger area. Further, such a merging event may also have caused a gas outflow perpendicular to the major axis of the galaxy which may also be the reason for the large measured source size.

When we compare the total power emitted in the $\mathrm{CO}(3-2)$ line with the size of the region in which it is produced, we find that the ratio of these two numbers is similar to the values found in the central regions of Maffei 2 and IC 342. Hence NGC 2146 is capable of maintaining physical gas conditions which are typical for starbursts over a large region, with a diameter of more than $2 \mathrm{kpc}$ and a thickness of more than $1 \mathrm{kpc}$.

Line Ratios. The velocity-integrated $\mathrm{CO}(3-2)$ intensity in the centre is $I_{\mathrm{CO}(3-2)}=172 \pm 17 \mathrm{~K} \mathrm{~km} \mathrm{~s}^{-1}$. For the $\mathrm{CO}(2-1)$ transition Xie et al. (1994) have measured $I_{\mathrm{CO}(2-1)}=123.3 \pm 27.4 \mathrm{~K} \mathrm{~km} \mathrm{~s}^{-1}$ at a similar angular resolution. Since their relatively large error includes also calibration uncertainties, it will only partly enter into our line ratio calculation, which yields a value of $R_{3,2}=1.4 \pm 0.2$ in the centre, and $1.0 \pm 0.2$ at a $30^{\prime \prime}$ offset along the major axis. For the $(2-1) /(1-0)$ line ratio Xie et al. give 
(c)

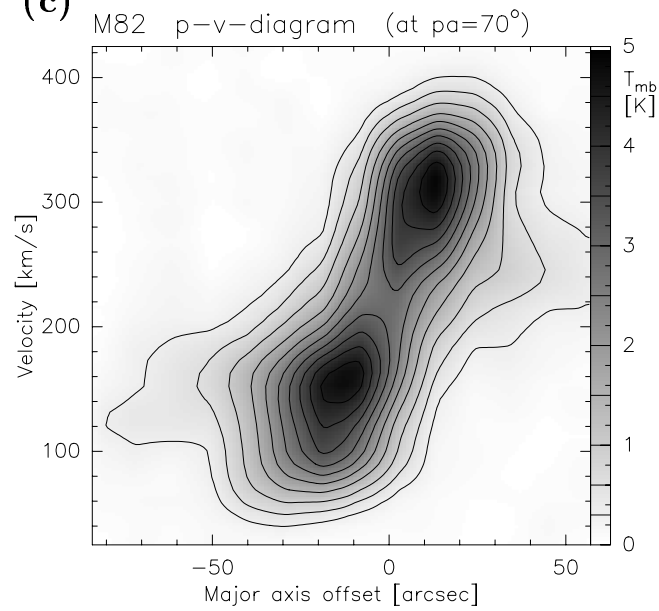

(d)

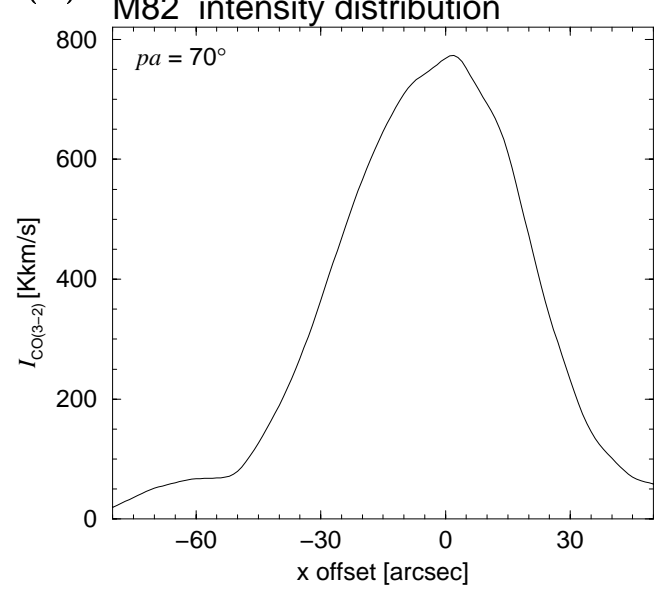

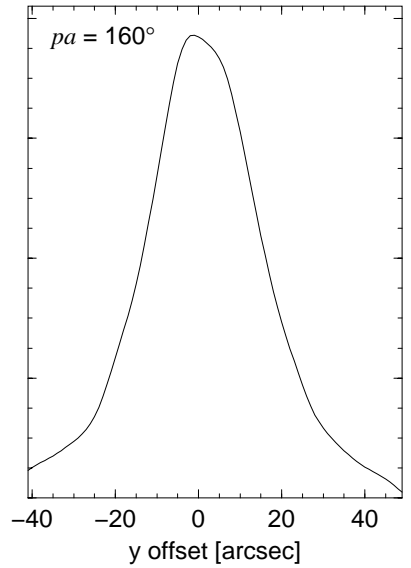

Fig. 7. c and d) $\mathrm{CO}(3-2)$ data of $\mathrm{M} 82$ : c) (left) position-velocity diagram along the major axis. Contours are 0.3, 0.6, 1.0, $1.5, \ldots 4.5 \mathrm{~K} . \mathrm{d}$ ) (right) intensity distribution along the major and minor axis, respectively. Note that for figure parts c) and d) the zero position differs from the position $(0,0)$ in figure parts a) and b) by $(\Delta \mathrm{RA}, \Delta$ Dec. $)=\left(-2^{\prime \prime} .0,4^{\prime \prime} .0\right)$.

$R_{2,1}=0.95 \pm 0.23$ in the centre (for a resolution of $45^{\prime \prime} \mathrm{HPBW}$. From these values we derive a value for the $(3-2) /(1-0)$ line ratio in the centre of this galaxy of $R_{3,1}=1.3 \pm 0.2$. This line ratio fits well with those of the other starburst galaxies in our sample, indicating similar densities and temperatures. Mauersberger et al. (1999) measured a slightly higher value for $I_{\mathrm{CO}(3-2)}$ and derived therefore a line ratio of $I_{3,1} \sim 1.6$.

Kinematics. The kinematics of the highly excited CO gas in NGC 2146 show rigid rotation with a large velocity gradient in the inner disk, reaching rotation velocities of $\pm 150 \mathrm{~km} \mathrm{~s}^{-1}$ at a radius of $15^{\prime \prime}$. The positionvelocity diagram along the minor axis, which is not shown here, shows further a large line width in the centre, indicating noncircular velocities. A fit to the central spectrum yields $\Delta V \sim 280 \mathrm{~km} \mathrm{~s}^{-1}$ and hence the largest linewidth among the galaxies in our sample. The line-shape, however, is clearly non-Gaussian. A two-component fit yields a line-width of about $130 \mathrm{~km} \mathrm{~s}^{-1}$ for the component that is more prominent in the southeastern half (with $V_{0} \sim 820 \mathrm{~km} \mathrm{~s}^{-1}$ ) and $150 \mathrm{~km} \mathrm{~s}^{-1}$ for the northwestern half (with $V_{0} \sim 990 \mathrm{~km} \mathrm{~s}^{-1}$ ).

All this points to NGC 2146 as a galaxy with violent star formation over a large fraction of its inner disk. Since this galaxy has no visible companion, the most reasonable explanation is a recent merger, which should be modelled with the help of interferometric line observations of $\mathrm{HI}$ and $\mathrm{CO}$.

\section{7. $M 82$}

The prototypical nearby starburst galaxy M 82 is classified as I0 and has an inclination of $81^{\circ}$. We assume a distance of $3.2 \mathrm{Mpc}$, consistent with the value given by Tammann \& Sandage (1968). It is one of the most frequently observed extragalactic sources, because of its relatively high intensity in practically all wavelength ranges.
Different transitions of CO were observed and analysed by several authors. Knapp et al. (1980) noted an unusual line ratio of $R_{2,1} \sim 2$ in the inner part of this galaxy (which has not been confirmed by more recent observations). Wild et al. (1990), Turner et al. (1990), and Lo et al. (1990) observed the $\mathrm{CO}(3-2)$ emission at selected positions in $\mathrm{M} 82$. A CO $(3-2)$ map of the inner disk $\left(r \leq 30^{\prime \prime}\right)$ was presented by Tilanus et al. (1991). The (4-3) transition has been observed by Güsten et al. (1993) and White et al. (1994). Recently the HHT was used for $\mathrm{CO}(7-6)$ observations of this object (Mao et al. 2000).

Morphology. In this paper we present the first $\mathrm{CO}(3-2)$ map of M 82 which covers the object out to a radius of more than $1^{\prime}(>900 \mathrm{pc})$. The $\mathrm{CO}(3-2)$ emission is strongly enhanced in the vicinity of the centre, but it is still detected at a high level $\left(I_{\mathrm{CO}(3-2)}=30-50 \mathrm{~K} \mathrm{~km} \mathrm{~s}^{-1}\right)$ out to the edges of the observed region. The double-peak structure seen in ${ }^{13} \mathrm{CO}(3-2)$ data and in maps of other transitions (Mao et al. 2000) is not visible in the total intensity map of the ${ }^{12} \mathrm{CO}(3-2)$ emission (Fig. 7b), but in the position-velocity diagram (Fig. 7c). This points to differences in the excitation conditions and/or optical depths in the molecular lines between the centre and the molecular ring structure seen in the two peaks.

The reference position for our observations was chosen on the IR intensity peak (Rieke et al. 1980; see Table 1). For the data analysis (Figs. 7c and d) we shifted the reference point by $(\Delta \mathrm{RA}, \Delta \mathrm{Dec})=.\left(-2^{\prime \prime} .0,4^{\prime \prime} .0\right)$ onto the measured $\mathrm{CO}(3-2)$ maximum, and the position angle was assumed to be $70^{\circ}$, as in Tilanus et al. (1991). This value lies between the optical position angle $\left(65^{\circ}\right)$ and that determined by Neininger et al. (1998) from their interferometric ${ }^{13} \mathrm{CO}$ map of the centre of M 82 .

The source size - deconvolved by the beam - is measured to be $46^{\prime \prime} \times 23^{\prime \prime}$, corresponding to a linear scale of $710 \mathrm{pc} \times 360 \mathrm{pc}$ at the distance of $\mathrm{M} 82$. This region is larger than in some other starburst galaxies in the 
(a) NGC3628 ${ }^{12} \mathrm{Co}(3-2)$
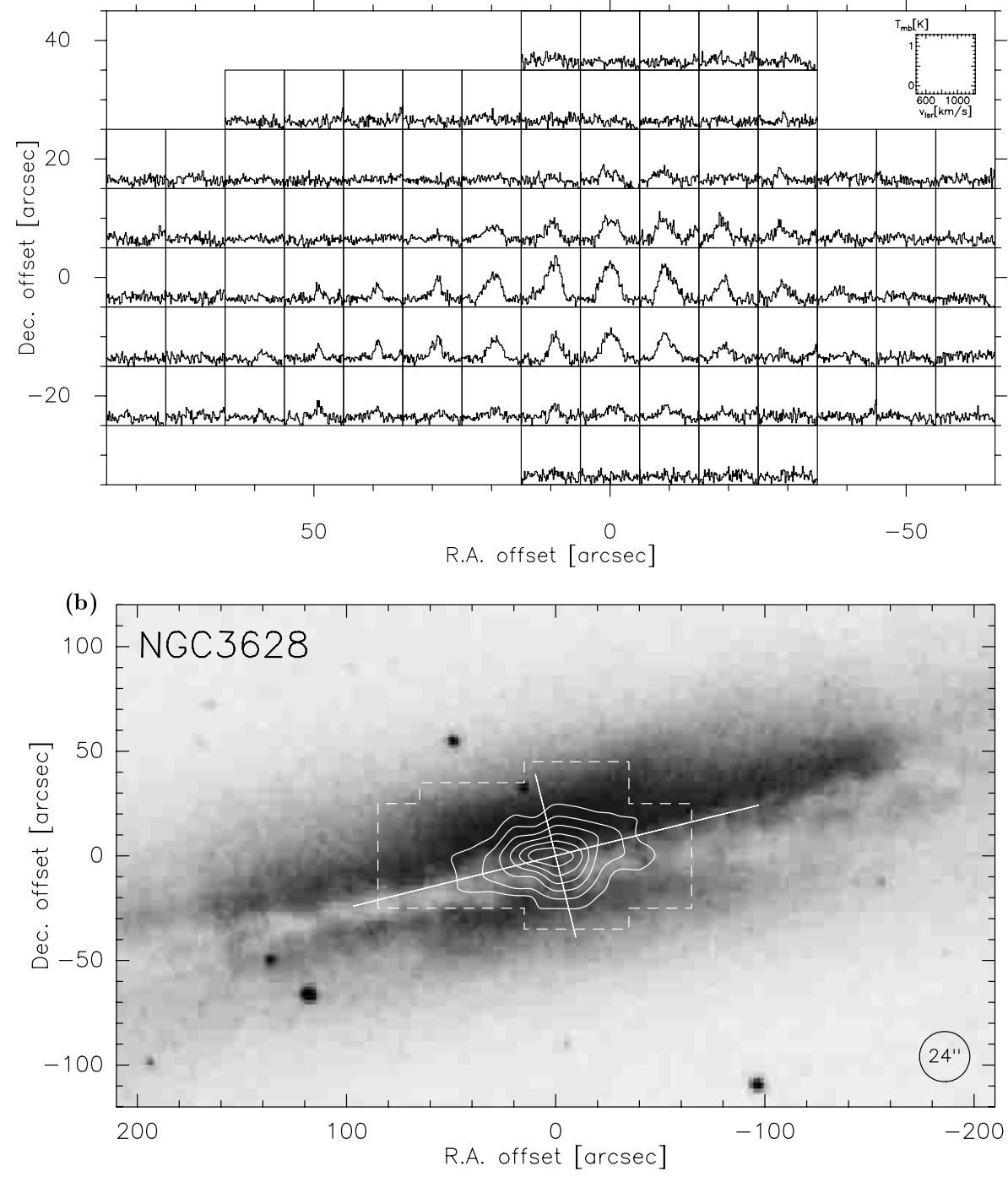

Fig. 8. a and b) $\mathrm{CO}(3-2)$ data of NGC 3628: a) (upper) raster map of the individual spectra, with $(0,0)$ corresponding to the position given in Table 1 . The scale of the spectra is indicated by the small box inserted in the upper right corner of the image. b) (lower) contour map of the integrated intensity, overlaid on an optical image extracted from the Digitized Sky Survey. Contour levels are 30, $60, \ldots 180 \mathrm{~K} \mathrm{~km} \mathrm{~s}^{-1}$. The region covered by the spectra shown in a) is indicated by the dashed polygon, and the cross shows the adopted centre and the direction of the major and minor axis. current sample, e.g. NGC 253 or Maffei 2. However, M 82 is not a spiral galaxy, but rather a small irregular, and rapid star formation could be triggered over a large fraction of this object by its interaction with M 81 .

Intensities and Line Ratios. M 82 shows the strongest $\mathrm{CO}(3-2)$ emission of all objects in our sample. Typical brightness temperatures are $T_{\mathrm{mb}}=4-5 \mathrm{~K}$. The total $\mathrm{CO}(3-2)$ line flux in the region covered by our map is $F_{\mathrm{CO}(3-2)}=14.63 \pm 0.40 \times 10^{4} \mathrm{Jy} \mathrm{km} \mathrm{s}^{-1}$; the total power emitted in this line is $P_{\mathrm{CO}(3-2)}=16.4 \pm 0.5 \times 10^{30} \mathrm{~W}$. The line intensity measured in the central region is $I_{\mathrm{CO}(3-2)}=$ $770 \pm 70 \mathrm{~K} \mathrm{~km} \mathrm{~s}^{-1}$. A direct comparison with the values given by Wild et al. (1990) and Tilanus et al. (1991) is difficult due to the different beam sizes. The $\mathrm{CO}(1-0)$ data of Wild et al., however, were observed with the same angular resolution as our $\mathrm{CO}(3-2)$ data and allow the determination of line ratios. In the central region, we find a value of $R_{3,1}=1.0 \pm 0.2$, which is consistent with the results presented by Tilanus et al. (1991). At a distance of $20^{\prime \prime}$ from the centre along the major axis, the line ratio drops to $0.8 \pm 0.2$. Note the different reference position of Wild et al. (1990). We will not discuss the physical properties of the gas in detail here, but refer instead to the numerous multi-line investigations already published (e.g. Weiß et al. 2001). We argue that a line ratio around unity in contrast to other starburst galaxies - may be due to optical depth effects in the CO lines; see the publications cited above for a dicussion including data from other isotopomers.

Kinematics. A Gaussian fit to the central spectrum yields a line width of $\Delta V \sim 230 \mathrm{~km} \mathrm{~s}^{-1}$. From the shape of the spectra, however, it is clear that we see, even in the centre, two components which represent a ring-like distribution. A two-component fit to the central spectrum yields two separate lines with widths of about 130 and $100 \mathrm{~km} \mathrm{~s}^{-1}$ and at central velocities of $V_{0} \sim 180 \mathrm{~km} \mathrm{~s}^{-1}$ and $V_{0} \sim 290 \mathrm{~km} \mathrm{~s}^{-1}$, respectively. The kinematical structure of M 82 as a whole is a bit more complicated. The two components visible in the central spectrum extend to radii of $\pm 30^{\prime \prime}$ on either side, where they rotate with a velocity of $\pm 80 \mathrm{kms}^{-1}$ relative to the systemic velocity of 
(c)

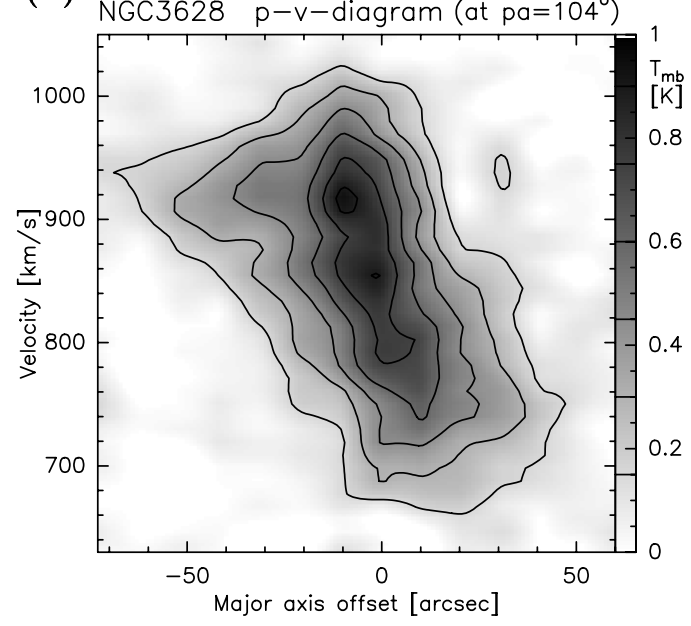

(d)

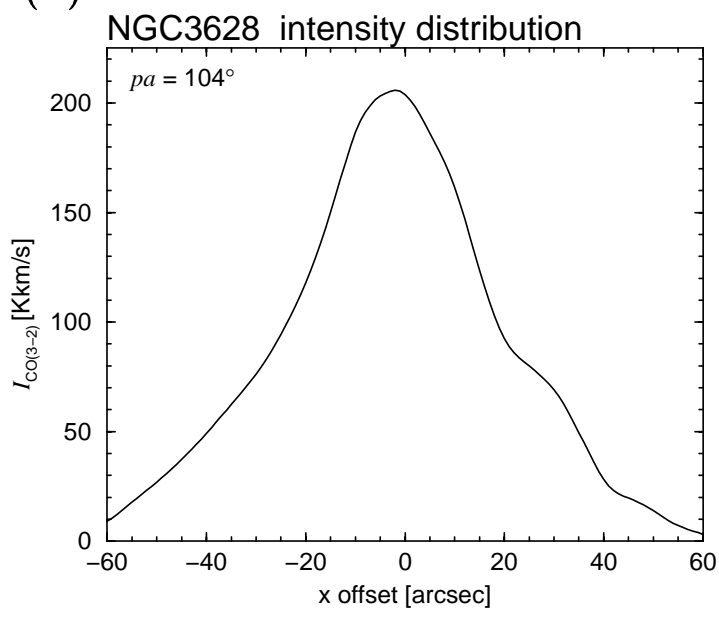

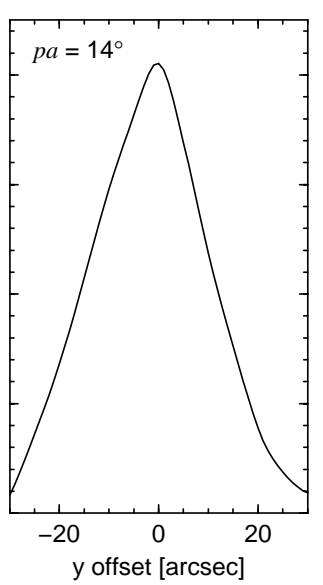

Fig. 8. c and d) $\mathrm{CO}(3-2)$ data of NGC 3628: c) (left) position-velocity diagram along the major axis. Contours are 0.15 , $0.3, \ldots 0.9 \mathrm{~K} . \mathbf{d})$ (right) intensity distribution along the major and minor axis, respectively.

$225 \mathrm{~km} \mathrm{~s}^{-1}$. From the velocity field (not shown) we conclude further that this rotation is in the plane of M 82 (i.e. along the major axis). The rotation above and below the plane seems to differ from this behaviour significantly. Below the plane, the measured velocities of the $\mathrm{CO}(3-2)$ emission are $<200 \mathrm{~km} \mathrm{~s}^{-1}$, probably indicating an outflow of highly excited molecular gas towards the observer in the southern halo of M 82 .

\subsection{NGC 3628}

The disturbed edge-on galaxy NGC 3628 is a member of the well known Leo triplet of galaxies and exhibits a starburst, which is probably triggered by the interaction of NGC 3628 with its neighbours NGC 3623 and NGC 3627. It is viewed edge-on with an inclination of $89^{\circ}$, and its distance is assumed here to $6.7 \mathrm{Mpc}$ (de Vaucouleurs 1975) which is consistent with previous studies of this object. Despite its starburst properties, it was not observed in the higher CO transitions before. Reuter et al. (1991) observed the $\mathrm{CO}(2-1)$ line in this object, while Boissé et al. (1987) observed several $\mathrm{CO}(1-0)$ spectra in the inner $2^{\prime}$. Interferometric $\mathrm{CO}$ observations were presented by Irwin \& Sofue (1996).

Morphology. The position of the CO maximum does not coincide with the optical centre, a fact that was already noted by Boissé et al. (1987) for the $\mathrm{CO}(1-0)$ line.

Even if the $\mathrm{CO}(3-2)$ emission in NGC 3628 shows a concentration towards the central region, the intensity distribution along the major axis is not as centrally peaked as for other starburst galaxies in our sample: the deconvolved source size is $34^{\prime \prime} \times 15^{\prime \prime}$, corresponding to a linear size of $1.1 \mathrm{kpc} \times 0.5 \mathrm{kpc}$ at a distance of $6.7 \mathrm{Mpc}$. This slower intensity decrease along the major axis indicates that violent star formation does not only occur in the very centre, but rather in a central bar or disk which is somewhat elongated along the major axis.
Intensities and Line Ratios. The integrated $\mathrm{CO}(3-2)$ line flux measured from NGC 3628 is $F_{\mathrm{CO}(3-2)}=2.68 \pm$ $0.14 \times 10^{4} \mathrm{Jy} \mathrm{km} \mathrm{s}^{-1}$. This corresponds, at an assumed distance of $6.7 \mathrm{Mpc}$, to a total power of $P_{\mathrm{CO}(3-2)}=$ $1.31 \pm 0.07 \times 10^{31} \mathrm{~W}$, which is of the same order as for M 82, but is emitted from a larger region. The maximum intensity measured in the central region of NGC 3628 is $I_{\mathrm{CO}(3-2)}=206 \pm 19 \mathrm{~K} \mathrm{~km} \mathrm{~s}^{-1}$. From the $\mathrm{CO}(1-0)$ data of Boissé et al. (1987), which are observed with a similar beam size, we estimate a line ratio of $R_{3,1}=1.4 \pm 0.2$. This is, together with M83, the highest value in the present sample. From a CO(2-1) map from Reuter et al. (1991), smoothed to $21^{\prime \prime}$ resolution, we can further determine $R_{3,2}=1.5 \pm 0.2$. For this galaxy the integrated intensity of the $\mathrm{CO}(2-1)$ line is smaller than that of both the (1-0) and the (3-2) transition in the central region. At radii larger than about $30^{\prime \prime}$, both line ratios decrease to $1.0 \pm 0.2$, similar to the other starburst objects in our sample.

In the centre, the high line ratio indicates a kinetic temperature of the $\mathrm{CO}$ gas of $T>60 \mathrm{~K}$, at molecular gas densities around $n\left(\mathrm{H}_{2}\right) \sim 10^{3.5} \mathrm{~cm}^{-3}$. The $\mathrm{CO}(3-2)$ spectrum presented by Mauersberger et al. (1999) was taken at a position nearly $25^{\prime \prime}$ off the centre toward the southeast. They estimated a line ratio of $R_{3,1} \sim 0.2$, which is below our value in the disk, and is not consistent with the starburst properties in the central region. This again shows that an extended mapping of the target sources is necessary for a detailed analysis of line ratios.

Kinematics. The kinematics as traced by the $\mathrm{CO}(3-2)$ emission are dominated by a large line width in the central region (see Fig. 8c), and by a large velocity gradient in the inner $20^{\prime \prime}$. Both results suggest either fast rotation or non-circular velocities near the centre. The latter may be a signature of a barred potential in the inner part of NGC 3628, feeding the starburst nucleus. The velocity field (not shown) shows some velocity shear between the southern and northern half. This may be a consequence 


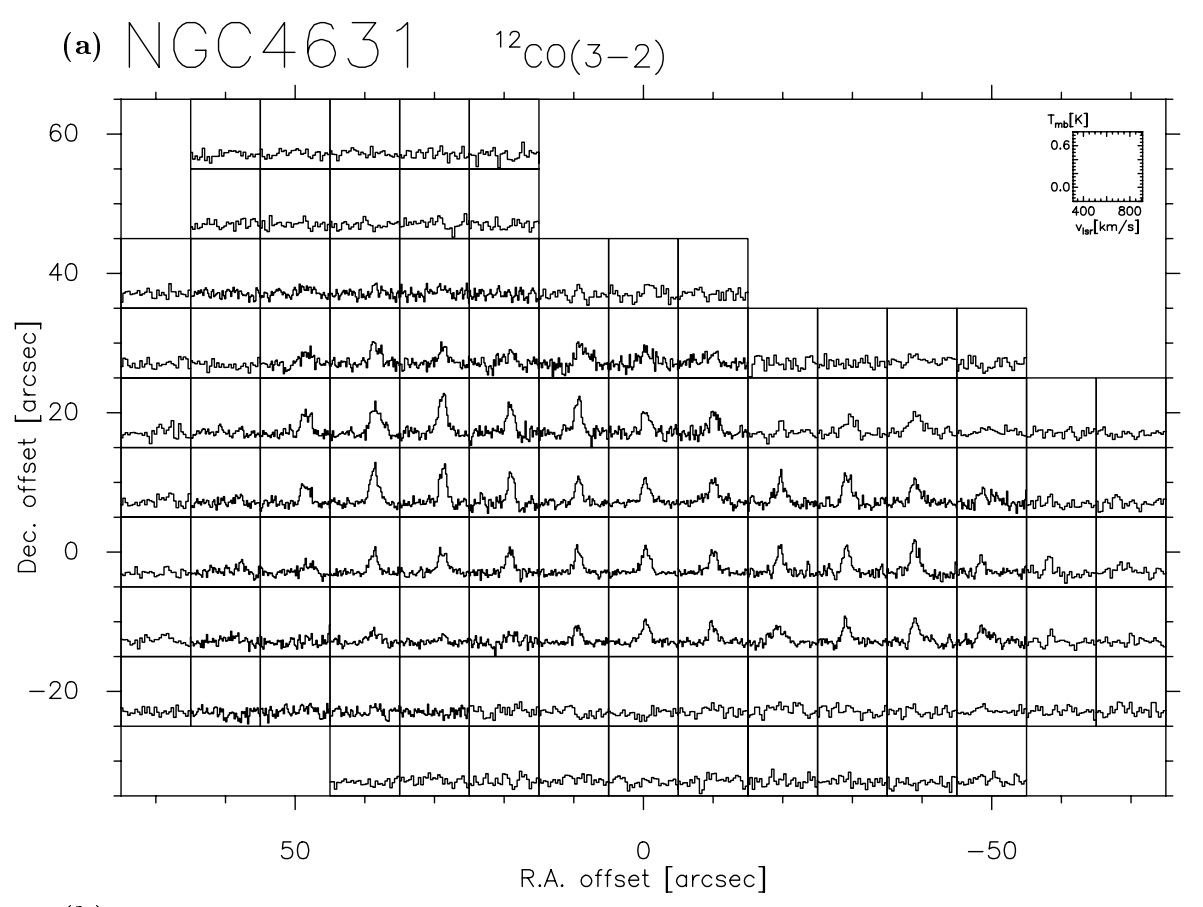

(b)

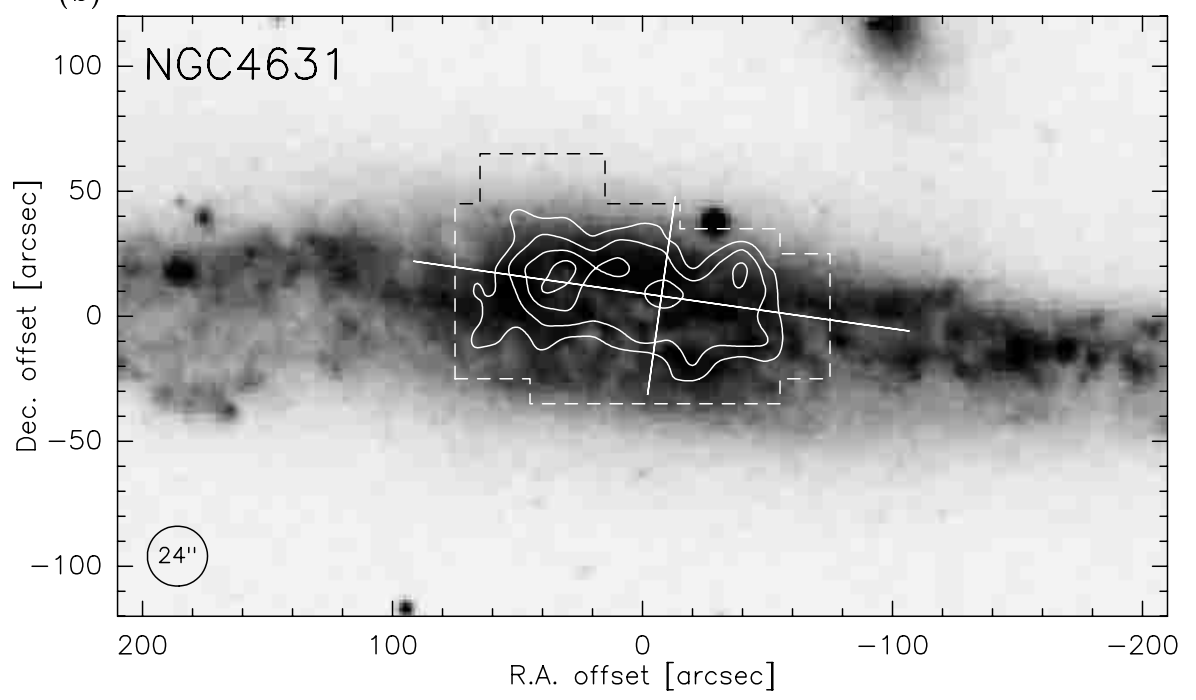

Fig. 9. a and b) $\mathrm{CO}(3-2)$ data of NGC 4631: a) (upper) raster map of the individual spectra, with $(0,0)$ corresponding to the position given in Table 1. The scale of the spectra is indicated by the small box inserted in the upper right corner of the image. b) (lower) contour map of the integrated intensity, overlaid on an optical image extracted from the Digitized Sky Survey. Contour levels are 11, 22, 33, $44 \mathrm{~K} \mathrm{~km} \mathrm{~s}^{-1}$. The region covered by the spectra shown in a) is indicated by the dashed polygon, and the cross shows the reference position for data analysis (see text) and the direction of the major and minor axis.

of the interaction between NGC 3628 and its neighbours NGC 3623 and NGC 3627 (Rots 1978).

\subsection{NGC 4631}

NGC 4631 is a disturbed, edge-on $\left(i \sim 86^{\circ}\right)$ late-type galaxy. It is a member of an interacting group of at least 3 galaxies which includes also NGC 4627 and NGC 4656 (e.g. Combes 1978). For a distance of $D=7.5 \mathrm{Mpc}$ (consistent with earlier publications, e.g. Golla \& Wielebinski 1994), the separation of NGC 4631 from its neighbours is about $60 \mathrm{kpc}$ and $4 \mathrm{kpc}$, respectively. As a consequence of this interaction, NGC 4631 shows a disturbed appearance in the optical and is believed to exhibit a mild starburst (Golla \& Wielebinski 1994). These authors have observed the $\mathrm{CO}(1-0)$ and the $\mathrm{CO}(2-1)$ line transitions with the IRAM $30 \mathrm{~m}$ telescope. Recently Neininger \& Dumke (1999) found intergalactic cold dust (traced by continuum emission at $1.2 \mathrm{~mm}$ ) in this galaxy group. This emission is connected with the $\mathrm{HI}$ spurs emerging from the disk (Rand 1994), probably as a consequence of the mentioned interaction. The $\mathrm{CO}(3-2)$ data we present here were published as a smoothed raster map of spectra in Wielebinski et al. (1999).

Morphology. The spatial distribution of the $\mathrm{CO}(3-2)$ emission (Fig. 9) differs from the other starburst galaxies in our sample, and shows that the starburst properties (if we follow the "mild starburst" classification of Golla \& Wielebinski 1994) are different from starbursts occuring in the nuclei of galaxies. Besides the central maximum with a velocity-integrated intensity of $I_{\mathrm{CO}(3-2)}=37 \pm$ $6 \mathrm{~K} \mathrm{~km} \mathrm{~s}^{-1}$ there are two further maxima at radii of about $\pm 40^{\prime \prime}$, which indicate a disk- or ring-like distribution of the CO gas emitting in the (3-2) line. The eastern peak of this ring, with an intensity of $I_{\mathrm{CO}(3-2)}=46 \pm 7 \mathrm{~K} \mathrm{~km} \mathrm{~s}^{-1}$, 
(c)

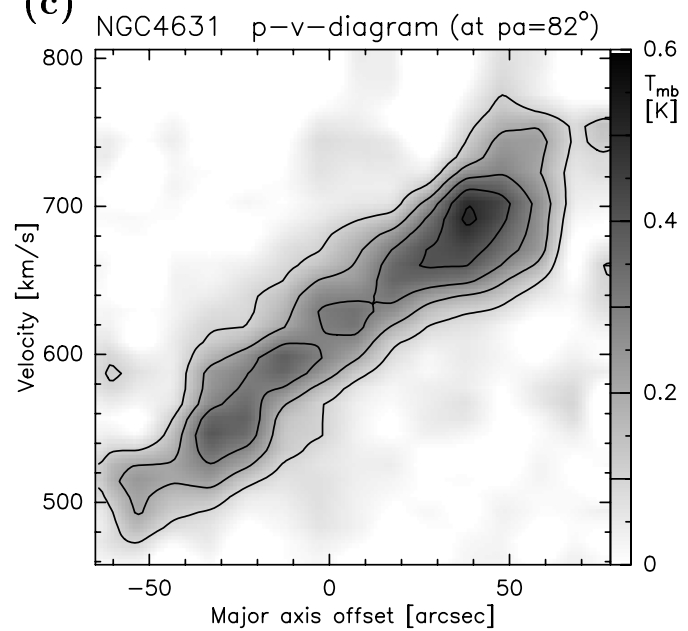

(d)

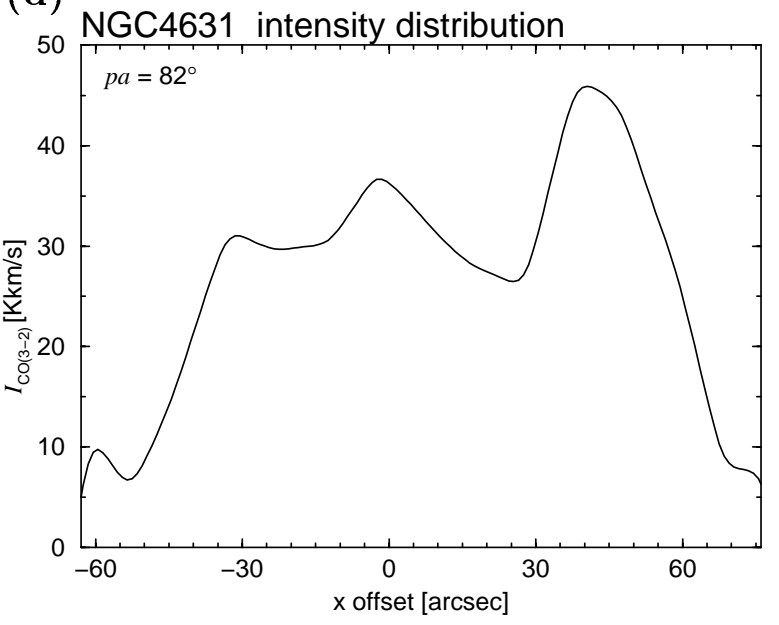

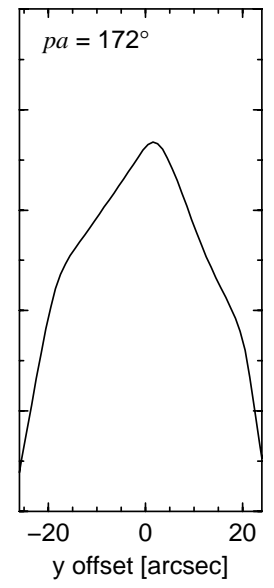

Fig. 9. c and d) $\mathrm{CO}(3-2)$ data of NGC 4631: c) (left) position-velocity diagram along the major axis. Contours are 0.1, $0.2, \ldots 0.5 \mathrm{~K} . \mathbf{d})$ (right) intensity distribution along the major and minor axis, respectively. Note that for figure parts c) and d) the zero position differs from the position $(0,0)$ in figure parts a) and b) by $(\Delta \mathrm{RA}, \Delta \mathrm{Dec})=.\left(-7^{\prime \prime} .6,8^{\prime \prime} .0\right)$.

corresponds to the star-forming region CM 67. Because of this distribution, we cannot estimate a central peak size here. Thus we estimate the size of the whole $\mathrm{CO}(3-2)$ emitting region. We find, assuming a disk-like morphology and after deconvolving in $z$-direction, a size of $100^{\prime \prime} \times 21^{\prime \prime}$. This corresponds to $3.63 \mathrm{kpc} \times 0.77 \mathrm{kpc}$, of which, however, only the latter value (the extent along the minor axis) can be compared with the other galaxies in our sample. As noted by Wielebinski et al. (1999), the detected $\mathrm{CO}(3-2)$ emission is as extended as the lower transitions observed by Golla \& Wielebinski (1994).

We note here that the intensity distributions (Fig. 9d) and the position-velocity diagram (Fig. 9c) are not relative to the position given in Table 1 , but to a reference position which is located at $(\Delta \mathrm{RA}, \Delta$ Dec. $)=\left(-7^{\prime \prime} .6,8^{\prime \prime} .0\right)$ in Figs. 9a and b. This was done in order to center the distributions on the $\mathrm{CO}(3-2)$ emission. Interestingly, this reference position is even $\sim 5^{\prime \prime}$ northwest of the central position given by Golla \& Wielebinski (1994), namely - after conversion to $\mathrm{J}(2000)$ coordinates $-\mathrm{RA}[2000]=12^{\mathrm{h}} 42^{\mathrm{m}} 07^{\mathrm{s}} .2$, Dec. $[2000]=32^{\circ} 32^{\prime} 32^{\prime \prime}$. Hence the $\mathrm{CO}(3-2)$ maxima seem to be slightly north of those for the lower transitions. This is possibly connected to the outflow of interstellar matter into the northern halo of NGC 4631, either by varying optical depth effects or changing gas properties with increasing distance from the major axis.

Intensities and Line Ratios. We measure a total line flux in NGC4631 of $F_{\mathrm{CO}(3-2)}=1.35 \pm 0.11 \times$ $10^{4} \mathrm{Jy} \mathrm{kms}^{-1}$, which corresponds to a total power of $P_{\mathrm{CO}(3-2)}=8.3 \pm 0.7 \times 10^{30} \mathrm{~W}$ emitted in the $\mathrm{CO}(3-2)$ line. Due to the different locations of the emission maxima for the different transitions, the line ratios vary for a constant galactocentric radius. When comparing our data with the $\mathrm{CO}(1-0)$ data from Golla \& Wielebinski (1994), we find values of $R_{3,1}$ between $0.9 \pm 0.2$ and $1.2 \pm 0.2$ in the central region and between $0.6 \pm 0.2$ and $0.9 \pm 0.3$ close to the outer maxima. The line ratio between the $(2-1)$ and the (1-0) transition is about $R_{2,1}=0.8-0.9$ for all intensity peaks. These line ratios indicate moderately warm molecular gas of $\sim 30-50 \mathrm{~K}$. We conclude that NGC 4631 is not an active starburst galaxy. However, in the molecular ring, at radii of about $\pm 40^{\prime \prime}$, there is some enhanced star formation taking place. This is also suggested by the $\mathrm{H} \alpha$ morphology (Golla et al. 1996). In addition, the huge radio halo visible at cm-wavelengths and the magnetic field orientation (e.g. Hummel et al. 1991; Golla \& Hummel 1994) point to some kind of activity in the central region. This activity has probably been triggered by the interaction of NGC 4631 with its neighbours.

Kinematics. The kinematics as obtained from the $\mathrm{CO}(3-2)$ emission do not show significant differences from data from the lower transitions. Rigid rotation can be seen out to radii of $40^{\prime \prime}-50^{\prime \prime}$. The line width in the central region is narrow compared to the starburst galaxies in our sample and also compared to the other edge-on objects, about $80 \mathrm{~km} \mathrm{~s}^{-1}$. This line width stays more or less constant along the major axis of NGC 4631. In the centre no fast rotating gas or noncircular velocities can be seen. The velocity field (not shown) shows further some kinematic anomalies in the northern half, also due to the interaction of NGC 4631 with its neighbours.

\subsection{0. $M 51$}

M 51 (NGC 5194) is a grand-design face-on $\left(i=20^{\circ}\right)$ spiral galaxy at a distance of $D=9.6 \mathrm{Mpc}$ (Sandage \& Tammann 1975). It has already been subject to several studies of the molecular line emission (e.g. Nakai et al. 1994; García-Burillo et al. 1993, and references therein). The data for this galaxy were shown by Wielebinski et al. (1999) to demonstrate that the $\mathrm{CO}(3-2)$ emission is also found in the disks of galaxies. Thus the physical conditions in the spiral arms also lead to a significant excitation of 

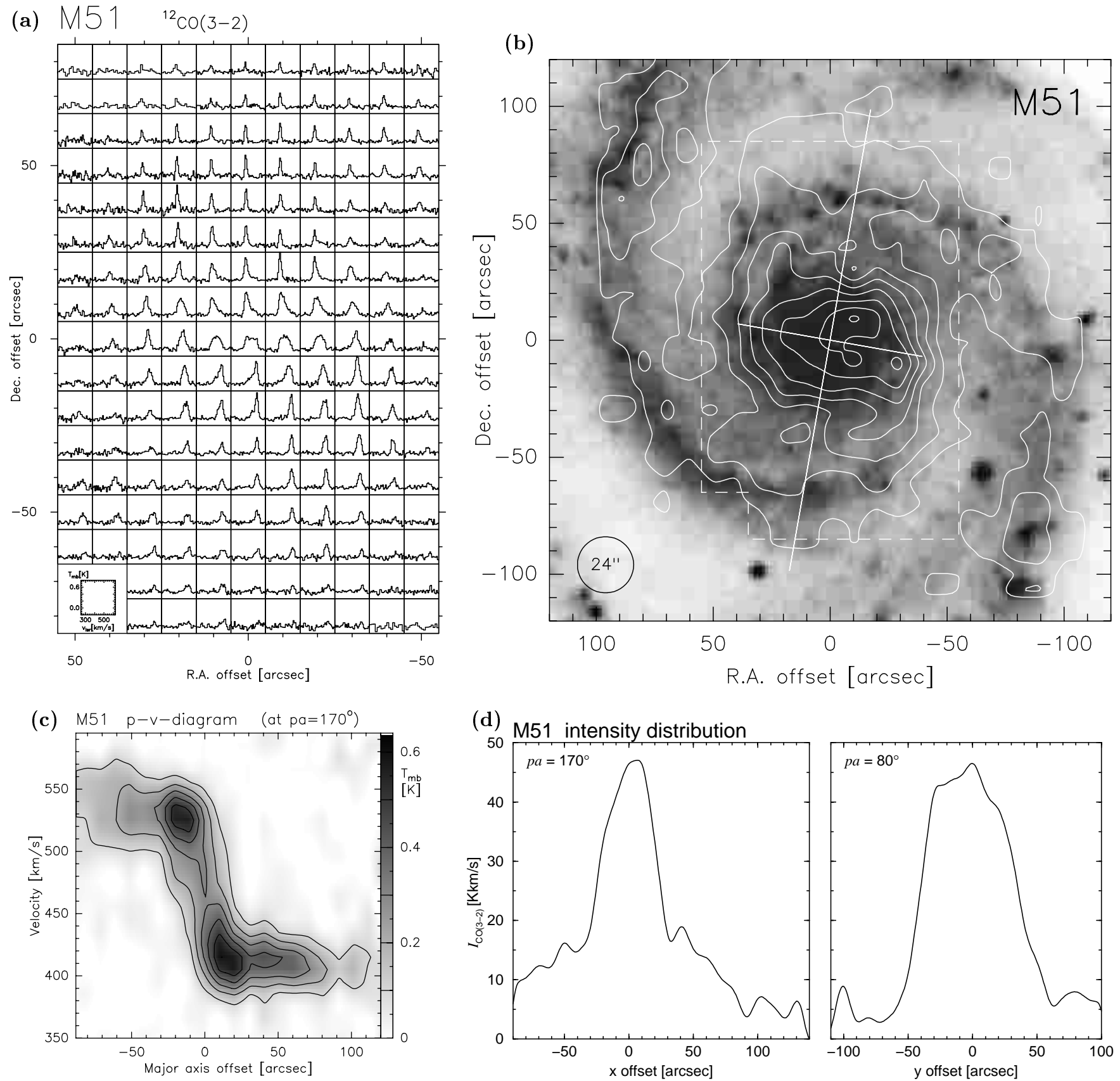

Fig. 10. $\mathrm{CO}(3-2)$ data of $\mathrm{M} 51$ : a) (upper left) raster map of the individual spectra, with $(0,0)$ corresponding to the position given in Table 1. The scale of the spectra is indicated by the small box inserted in the lower left corner of the image. Only the inner part of the mapped area is shown here. b) (upper right) contour map of the integrated intensity, overlaid on an optical image extracted from the Digitized Sky Survey. Contour levels are $6,12,18, \ldots, 48 \mathrm{~K} \mathrm{~km} \mathrm{~s}^{-1}$. The region covered by the spectra shown in a) is indicated by the dashed polygon, and the cross shows the adopted centre and the direction of the major and minor axis. c) (lower left) position-velocity diagram along the major axis. Contours are $0.1,0.2, \ldots 0.6 \mathrm{~K}$. d) (lower right) intensity distribution along the major and minor axis, respectively. Note that while only the spectra in the inner part of M 51 are shown in a), the other figure parts include the whole data set as shown by Wielebinski et al. (1999).

the $J=3$ level of $\mathrm{CO}$. In the present paper we only show the spectra of the inner disk of M 51. The distribution of the $\mathrm{CO}(3-2)$ emission in the spiral arms can be seen in Fig. 10b, which shows an overlay of our $\mathrm{CO}(3-2)$ intensity map, as obtained from the complete data set, on an optical image.
Morphology. Even though the $\mathrm{CO}(3-2)$ emission is seen in the spiral arms, emission from the centre dominates, with a maximum intensity of $I_{\mathrm{CO}(3-2)}=47 \pm 7 \mathrm{~K} \mathrm{~km} \mathrm{~s}^{-1}$. This is a factor 3 brighter than the maxima in the spiral arms. Interestingly the extent of the central peak is larger along the minor axis than along the major axis (the 
position angle can easily be estimated from the isovelocity contours in the velocity field); the deconvolved source size is $47^{\prime \prime} \times 76^{\prime \prime}$. This corresponds to $2.2 \mathrm{kpc} \times 3.5 \mathrm{kpc}$ at a distance of $9.6 \mathrm{Mpc}$. Another difference between M 51 and most other objects is the smooth transition between the line emitting central region and the spiral arms. For most galaxies in this survey the $\mathrm{CO}(3-2)$ emission drops to almost zero outside the central area, before it rises again within the spiral arms, as in M 83 (Fig. 11b) or NGC 6946 (Fig. 12b and Nieten et al. 1999). This connection between the highly excited CO gas in the centre and the disk is further seen only in NGC 891.

Intensities and Line Ratios. The total line flux measured for the central peak of $\mathrm{M} 51$ is $F_{\mathrm{CO}(3-2)}=1.6 \pm 0.2 \times$ $10^{4} \mathrm{Jy} \mathrm{km} \mathrm{s}^{-1}$, the total power radiated from the central region in the $\mathrm{CO}(3-2)$ line $P_{\mathrm{CO}(3-2)}=1.6 \pm 0.2 \times 10^{31} \mathrm{~W}$. The latter value is at the higher end of the range represented by our galaxy sample. When we normalize this value by the size of the emitting region, M51 shows a rather average result.

Wielebinski et al. (1999) compared the present data with the $\mathrm{CO}(1-0)$ observations of Nakai et al. (1994) and determined relatively low line ratios of $R_{3,1}=0.5 \pm 0.2$ in the central region and $R_{3,1}=0.7 \pm 0.2$ in the spiral arms. They observed, however, even higher values in the outermost spiral arms $\left(R_{3,1}=1.2 \pm 0.35\right)$. The ratio between the $(2-1)$ and the (1-0) transition was estimated by García-Burillo et al. (1993) to be almost constant over their map, $R_{2,1} \sim 0.8$ (with a similar angular resolution), but slightly higher in the centre. This leads to line ratios of $R_{3,2} \sim 0.65$ in the centre and $\sim 0.9$ in the spiral arms. This result is not consistent with that determined by GarcíaBurillo et al. (1993) from a few positions observed in the $\mathrm{CO}(3-2)$ line with the $30 \mathrm{~m}$ telescope. The difference, however, may be explained by the smaller beam size and the error beam of the $30 \mathrm{~m}$ telescope. The estimated values for $R_{3,1}$ are - beside NGC 891 - the lowest in our sample, and the inferred properties of the molecular gas are thus similar to NGC 891. Densities are $\sim 10^{3} \mathrm{~cm}^{-2}$ or below; and temperatures are in the range $20-40 \mathrm{~K}$.

Kinematics. The kinematical structure of the inner disk of M 51, as revealed by the position-velocity diagram and the velocity field, is similar to the $\mathrm{CO}(2-1)$ data from García-Burillo et al. (1993). Along the major axis the gas rotates rigidly out to a radius of $15^{\prime \prime}-20^{\prime \prime}$, and with a constant velocity further out. In the $p$ - $v$-diagram (Fig. 10c), the second and third temperature maximum, which are clearly seen in the $\mathrm{CO}(2-1)$ data at $r \sim 50^{\prime \prime}$ and $r \sim 120^{\prime \prime}$ respectively, are less pronounced (or even missing) in the $\mathrm{CO}(3-2)$ emission.

From a Gaussian fit to the central profile, we obtain a line width of $\Delta V \sim 120 \mathrm{~km} \mathrm{~s}^{-1}$. Since the profile is composed of two components, an appropriate fit yields line widths of $\sim 60 \mathrm{~km} \mathrm{~s}^{-1}$ for these components at central velocities of 430 and $500 \mathrm{~km} \mathrm{~s}^{-1}$.

\subsection{1. $M 83$}

M 83 is a barred galaxy at a distance of $D=4.7 \mathrm{Mpc}$ (Tully 1988). With an inclination of $i=24^{\circ}$ (Comte 1981) it is oriented relatively face-on. It is generally believed to undergo a nuclear starburst which is triggered by gas infall along the bar potential (Talbot et al. 1979).

Observations of the $\mathrm{CO}(1-0)$ line were performed basically in the spiral arms of this large nearby galaxy. Handa et al. (1990) made single-dish observations of the central region with an angular resolution of $16^{\prime \prime} \mathrm{HPBW}$. Petitpas $\&$ Wilson (1998) observed the inner $\pm 20^{\prime \prime}$ in the (3-2) and the (4-3) transition of CO.

Morphology. M 83 was also observed at larger radii from the centre within the spiral arms during our observing sessions (Thuma et al., in prep.), but we will restrict the discussion here to the emission from the central part of this galaxy. The line temperature reaches a maximum of $T_{\mathrm{mb}} \sim 2.3 \mathrm{~K}$, a value which is consistent with the JCMT observations of the $\mathrm{CO}(3-2)$ line of Petitpas \& Wilson (1998), considering their smaller telescope beam (and higher noise). The emission is concentrated to the nucleus, but also slightly elongated along the bar. The deconvolved extent of the central peak is $25^{\prime \prime} \times 23^{\prime \prime}$, corresponding to a linear size of $580 \mathrm{pc} \times 520 \mathrm{pc}$ at a distance of $4.7 \mathrm{Mpc}$.

Intensities and Line Ratios. The maximum intensity measured on the central peak is $I_{\mathrm{CO}(3-2)}=234 \pm$ $14 \mathrm{~K} \mathrm{~km} \mathrm{~s}^{-1}$. This is also consistent with the data of Petitpas \& Wilson (1998) - note that we are not able to resolve the double peak structure along the bar given the spatial resolution of our data. Even if we account for the slightly different reference position used by Mauersberger et al. (1999) and the large intensity gradient near the centre, the value of $I_{\mathrm{CO}(3-2)}=126 \mathrm{~K} \mathrm{~km} \mathrm{~s}^{-1}$ given by these authors underestimates the emission by about $25 \%$. The total line flux from the region shown in Fig. 11a can be determined to $F_{\mathrm{CO}(3-2)}=3.12 \pm 0.15 \times 10^{4} \mathrm{Jy} \mathrm{km} \mathrm{s}^{-1}$, which corresponds to a total power of $P_{\mathrm{CO}(3-2)}=7.5 \pm$ $0.4 \times 10^{30} \mathrm{~W}$. From Fig. 11d we estimate that a fraction of $10-20 \%$ of this emission does not originate in the central peak, but in an underlying molecular bar.

Handa et al. (1990) measured a CO(1-0) intensity near the centre of $I_{\mathrm{CO}(1-0)} \sim 180 \mathrm{~K} \mathrm{~km} \mathrm{~s}^{-1}$ (converted to $T_{\mathrm{mb}}$ ). Given the smaller beam size of their data, this can be used to estimate a lower limit for the line ratio of $R_{3,1}>1.2$. However, since these authors give a fully sampled $\mathrm{CO}(1-0)$ map, we can simulate a measurement with a larger beamsize by averaging the intensity values in the inner $30^{\prime \prime}$ of their map with an appropriate weighting. In this way we get a line ratio of $R_{3,1}=1.4 \pm 0.3$ (the large error accounts for the uncertainties of this method). In the disk, at radii between $30^{\prime \prime}$ and $50^{\prime \prime}$, the line ratio goes down to $0.9 \pm 0.3$. Therefore M 83 seems to have similar gas properties to most other galaxies in our sample.

Kinematics. The $p$-v-diagram along the major axis (Fig. 11c) shows a superposition of two kinematical structures. In the inner $\pm 15^{\prime \prime}$ we see a large velocity gradient 


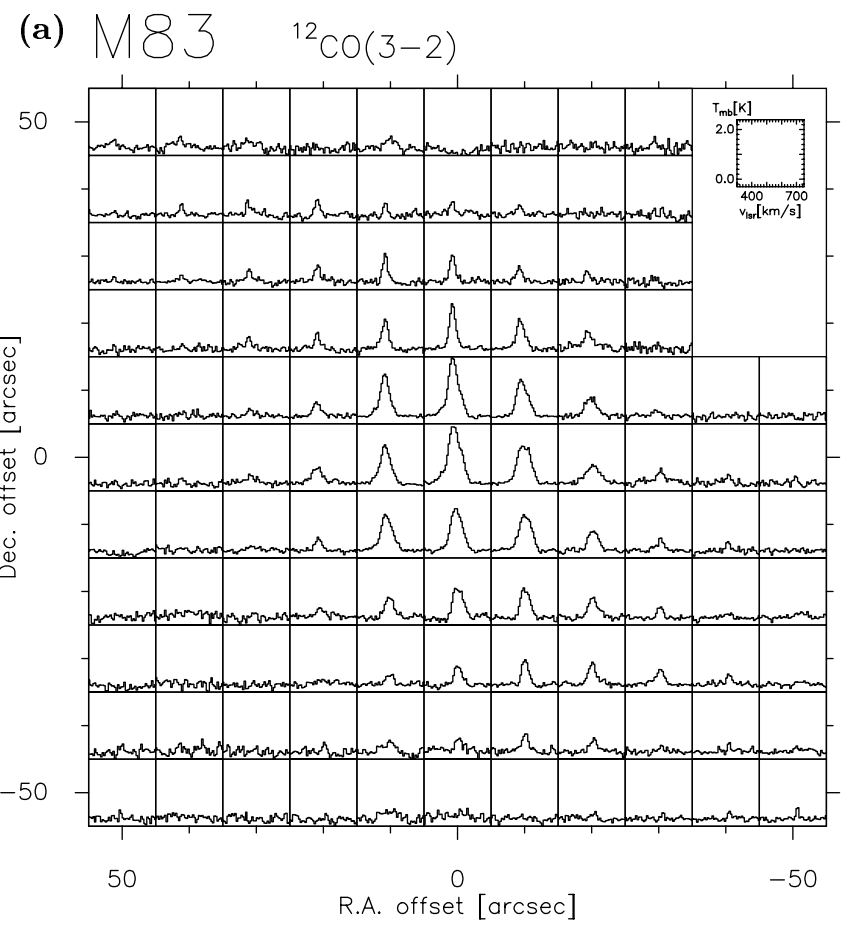

(b)
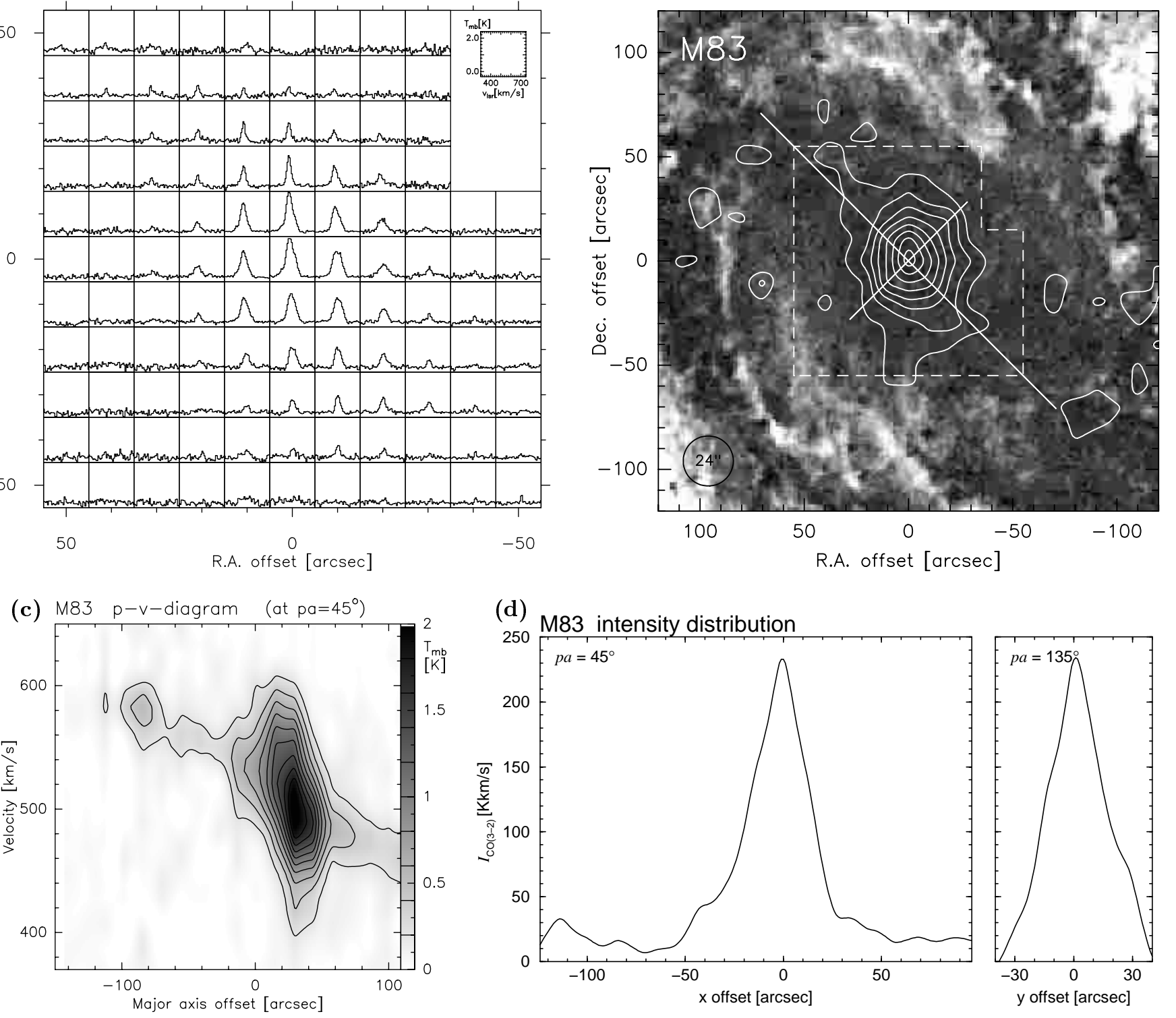

Fig. 11. $\mathrm{CO}(3-2)$ data of $\mathrm{M} 83:$ a) (upper left) raster map of the individual spectra, with $(0,0)$ corresponding to the position given in Table 1 . The scale of the spectra is indicated by the small box inserted in the upper right corner of the image. Only the inner part of the mapped area is shown here. b) (upper right) contour map of the integrated intensity, overlaid on an optical image extracted from the Digitized Sky Survey. Contour levels are 25, 50, 75, 100, 130, .., $220 \mathrm{~K} \mathrm{~km} \mathrm{~s}^{-1}$. The region covered by the spectra shown in a) is indicated by the dashed polygon, and the cross shows the adopted centre and the direction of the major and minor axis. c) (lower left) position-velocity diagram along the major axis. Contours are 0.2, 0.4, . 1.8 K. d) (lower right) intensity distribution along the major and minor axis, respectively. Note that while only the spectra in the inner part of M 83 are shown in $\mathbf{a}$ ), the other figure parts include the whole data set (see text).

from the systemic velocity of $510 \mathrm{~km} \mathrm{~s}^{-1}$ up to a rotational velocity of $\sim \pm 80 \mathrm{~km} \mathrm{~s}^{-1}$ relative to $v_{\text {sys }}$. This signature in the $p$ - $v$-diagram may be due to the $x_{2}$ orbits caused by the potential of the stellar bar which can be seen in optical images. A second kinematical system, probably due to the $x_{1}$ orbits of the mentioned bar, shows a smaller velocity gradient along the line defined by the position angle of M 83, reaching the same rotational velocity, but at a distance of about $\pm 120^{\prime \prime}$ from the centre. Near the centre we measure a line width of $\Delta V \sim 100 \mathrm{~km} \mathrm{~s}^{-1}$.

\subsection{NGC 6946}

NGC 6946 is a nearby grand-design spiral galaxy, oriented nearly face-on $\left(i=30^{\circ}\right)$ and classified as $\mathrm{SAB}(\mathrm{rs}) \mathrm{cd}$. Distance estimates for NGC 6946 range from 3.2 to $11 \mathrm{Mpc}$. De Vaucouleurs (1979) gives $5.1 \mathrm{Mpc}$, while Ferguson et al. (1998) use 5.3 Mpc. We will assume a distance of $5.2 \mathrm{Mpc}$ here.

NGC 6946 is believed to undergo a moderate starburst in its nucleus (Turner \& Ho 1983). It has already been 
(a) NGC6946 ${ }^{12} \mathrm{co}(3-2)$

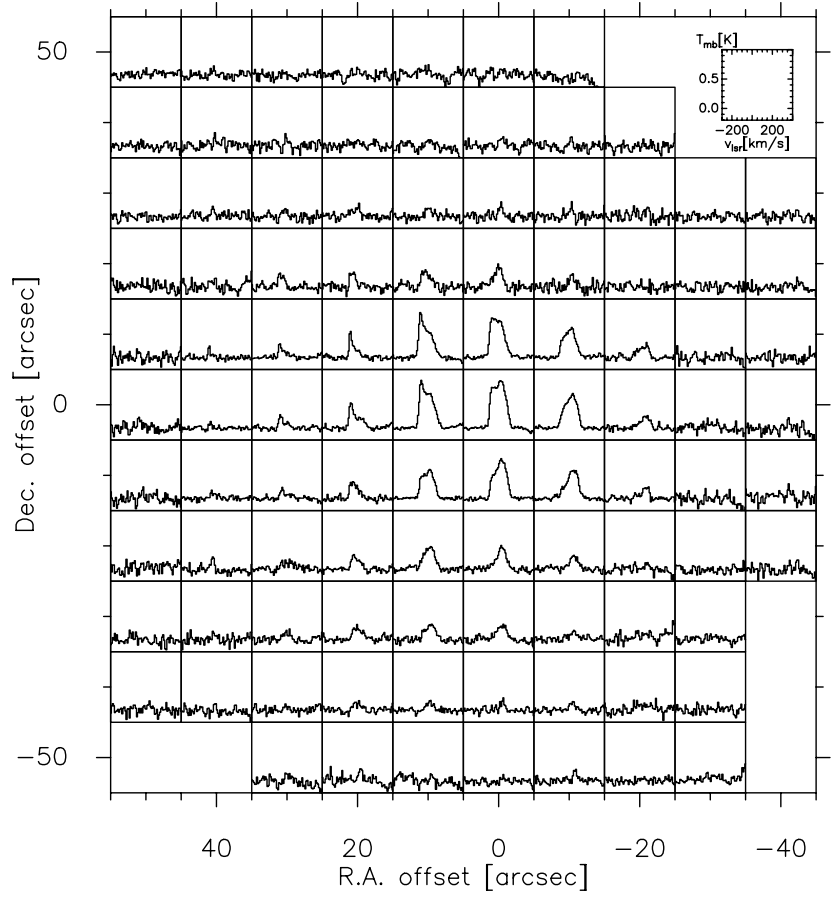

(c) NGC6946 $p-v$-diagram (at $\left.p a=60^{\circ}\right)$

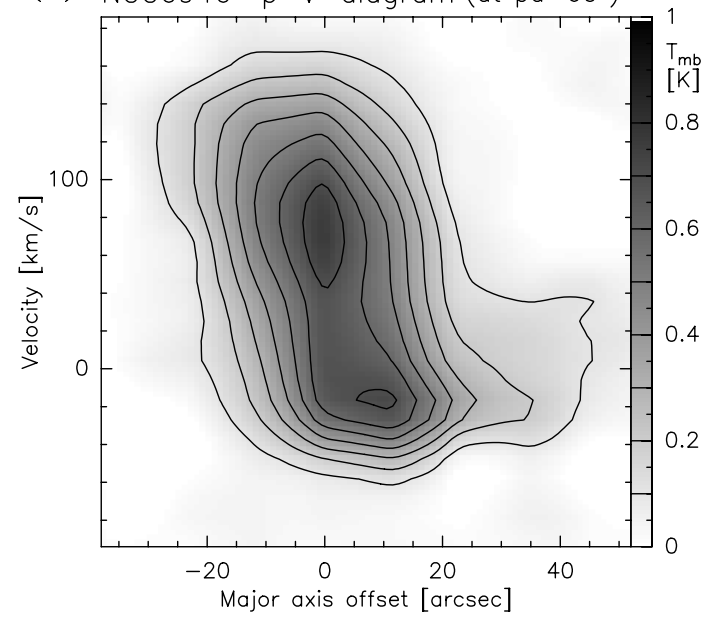

(d) (b)

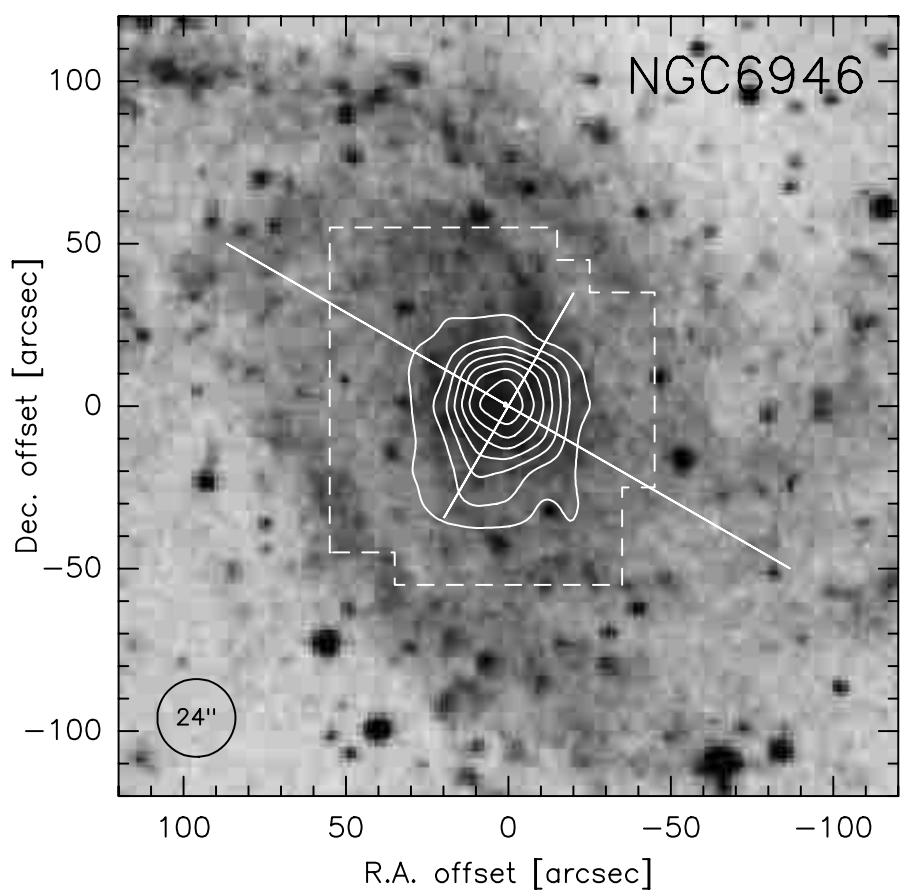

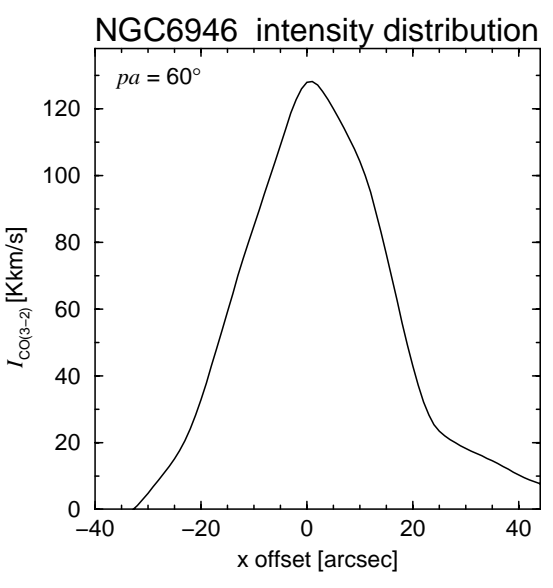

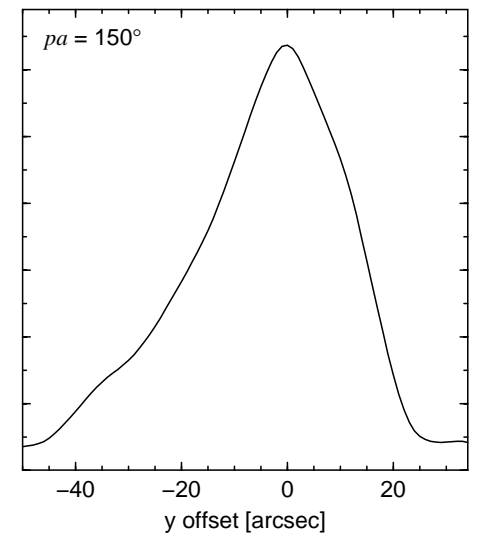

Fig. 12. $\mathrm{CO}(3-2)$ data of NGC 6946: a) (upper left) raster map of the individual spectra, with $(0,0)$ corresponding to the position given in Table 1. The scale of the spectra is indicated by the small box inserted in the upper right corner of the image. b) (upper right) contour map of the integrated intensity, overlaid on an optical image extracted from the Digitized Sky Survey. Contour levels are $16,32,48, \ldots, 128 \mathrm{~K} \mathrm{~km} \mathrm{~s}^{-1}$. The region covered by the spectra shown in a) is indicated by the dashed polygon, and the cross shows the adopted centre and the direction of the major and minor axis. c) (lower left) position-velocity diagram along the major axis. Contours are $0.1,0.2, \ldots 0.8 \mathrm{~K} . \mathrm{d}$ ) (lower right) intensity distribution along the major and minor axis, respectively.

studied at several wavelengths, but due to its large optical size there is no complete map in one of the $\mathrm{CO}$ transitions. While Sofue et al. (1988) and Weliachew et al. (1988) observed the $\mathrm{CO}(1-0)$ transition only in the central region, Young \& Scoville (1982) obtained some data along two orthogonal strips. Casoli et al. (1990) added some information about two regions in spiral arms $\pm 150^{\prime \prime}$ from the centre in the $\mathrm{CO}(1-0)$ and $(2-1)$ lines. The first results for higher $\mathrm{CO}$ transitions were presented by Nieten et al. (1999). These authors observed the $\mathrm{CO}(4-3)$ line in the inner part, using the HHT. While a few selected $\mathrm{CO}(3-2)$ spectra were already shown in that publication, here we present the full map of the inner disk of NGC 6946. We note that in this galaxy $\mathrm{CO}(3-2)$ emission is also found in the spiral arms, more than $3^{\prime}$ away from the centre. More extensive mapping of these off-centre regions in the $\mathrm{CO}$ lines has been performed by Walsh et al. (in prep.).

Morphology. Emission from the inner disk of NGC 6946 is concentrated to the nucleus. This result was also found by Weliachew et al. (1988), with a similar beam size, in the 
$\mathrm{CO}(1-0)$ line. The $\mathrm{CO}(3-2)$ data show a slightly larger extent to the south-southeast and a large intensity gradient in the opposite direction (see Figs. 12b and d). However, the distribution is not point-like - the deconvolved source extent is $19^{\prime \prime} \times 21^{\prime \prime}$ along the major and minor axis respectively, corresponding to $480 \mathrm{pc} \times 530 \mathrm{pc}$ at the distance of NGC 6946.

The brightness temperatures measured in the central region are about $T_{\mathrm{mb}}=0.8 \mathrm{~K}$. The $\mathrm{CO}(3-2)$ spectrum shown by Mauersberger et al. (1999) is a factor of 2 weaker. Their observing position, however, is located at $\left(-5^{\prime \prime},+10^{\prime \prime}\right)$ relative to our central coordinates, hence the results are not inconsistent.

Intensities and Line Ratios. The total $\mathrm{CO}(3-2)$ line flux from the central region of NGC 6946 is $F_{\mathrm{CO}(3-2)}=$ $1.42 \pm 0.07 \times 10^{4} \mathrm{Jy} \mathrm{km} \mathrm{s}^{-1}$, the total power emitted in this line is $P_{\mathrm{CO}(3-2)}=4.2 \pm 0.2 \times 10^{30} \mathrm{~W}$. From our data we calculate a maximum integrated line intensity of $I_{\mathrm{CO}(3-2)}=129 \pm 10 \mathrm{Kkms}^{-1}$. By comparing this value with the $\mathrm{CO}(1-0)$ data from Weliachew et al. (1988), we estimate a line ratio of $R_{3,1}=1.3 \pm 0.2$ in the central region. Further we find $R_{3,1}=1.0 \pm 0.2$ at a distance of $30^{\prime \prime}$ from the centre. These values are again similar to those for other starburst galaxies in our sample.

Kinematics. The kinematics in the inner region of NGC 6946, as traced by CO(3-2), show no differences from the properties found by Weliachew et al. (1988) in the $\mathrm{CO}(1-0)$ line. We see a steep velocity gradient and a large line width in the centre. From a Gaussian fit we obtain a line width of $\Delta V \sim 150 \mathrm{~km} \mathrm{~s}^{-1}$ for the central spectrum.

\section{Discussion and conclusions}

\subsection{General properties and activity}

For the target galaxies we collect some parameters from the literature that are of interest when comparing the galaxies in Table 2. These include some results from IR measurements which can be used as a tracer for the star forming activity. However, care must be taken because these values were obtained using IRAS and are therefore global values, while the $\mathrm{CO}(3-2)$ data usually refer to the central regions of the target galaxies. These data include the IR color, $S_{60} / S_{100}$, which is the ratio of the measured flux densities at 60 and $100 \mu \mathrm{m}$ respectively. This is correlated with the dust temperature in the galaxies. The IR luminosity, $L_{\mathrm{IR}}$, also depends on the dust temperature, but is, according to Young et al. (1989), more closely related to the total molecular mass as given by $\mathrm{CO}(1-0)$ measurements. This explains the high IR luminosity for spiral galaxies which form stars because of their large amount of molecular gas (e.g. NGC 891 and M 51) and not because of peculiar physical properties. We also give the assumed distance to each galaxy in Table 2 , since some determined parameters (luminosities, masses, sizes) are distance dependent.

\subsection{Kinematics}

As already mentioned, the $\mathrm{CO}(3-2)$ line traces a different component of the molecular gas in galaxies than the lower transitions. The kinematic properties of this component may differ from the bulk of cold molecular gas, and from other ISM components.

In general, the largest line widths are seen in the edgeon galaxies. Here they reflect the rigid rotation of the inner gas disk, as well as possible non-circular motions. In our sample, the objects classified as starbursts show the largest linewidths: up to $280 \mathrm{~km} \mathrm{~s}^{-1}$ for NGC 2146. The normal galaxy NGC 891 and NGC 4631 (sometimes called a "mild starburst") show only linewidths of $100 \mathrm{~km} \mathrm{~s}^{-1}$ and $80 \mathrm{~km} \mathrm{~s}^{-1}$ in their central regions, respectively. Note that these values are not corrected for inclination, otherwise the difference between NGC 2146 and the other galaxies would be even larger.

For galaxies which are oriented close to face-on, the measured linewidth is caused by the velocity dispersion of the gas. This dispersion is expected to depend on the star-forming activity. The smallest values are measured for NGC $278\left(40 \mathrm{~km} \mathrm{~s}^{-1}\right)$ and IC $342\left(60 \mathrm{~km} \mathrm{~s}^{-1}\right)$, while all other face-on objects show values between 100 and $150 \mathrm{~km} \mathrm{~s}^{-1}$.

\subsection{Extended emission}

One of the most important results of this investigation is that the $\mathrm{CO}(3-2)$ emission - and hence the warm and dense gas - is not restricted to the nuclei of the galaxies as suggested earlier (e.g. Mauersberger et al. 1996), a fact already emphasized by Wielebinski et al. (1999). This shows the necessity of extended mapping also in the higher CO line transitions. To better quantify the extent of the emission and to set the $\mathrm{CO}(3-2)$ morphology in relation to the general properties and types of the observed galaxies, we investigate the extended gas in more detail.

From the intensity distribution we estimated the source size by fitting a Gaussian to the central emission peak, and deconvolving it by the (very simple) assumption that the intrinsic (unconvolved) intensity distribution is also Gaussian. For most of the galaxies the central emission peak could be reasonably well fitted by a Gaussian with an error for the estimated width of about $\pm 2^{\prime \prime}$. For NGC 278 and NGC 4631, the extent was estimated by the assumption that the emission is distributed disk-like. Here the error is about $\pm 4^{\prime \prime}$. See also the individual subsections of Sect. 3 for details. The results are listed in Table 3, for the measured (in ") and the deconvolved (in kpc) size.

The sizes of the central emission peak vary between $300 \mathrm{pc}$ and more than $3 \mathrm{kpc}$ between the galaxies. There is no correlation between the peak size and other properties of the galaxies. Among the edge-on galaxies, however, the disturbed starburst galaxy NGC 2146 shows a very large extent along the minor axis $(\sim 1.4 \mathrm{kpc})$, which is probably connected to the peculiar optical appearance and the high star formation in this galaxy. 
Table 2. General source properties obtained from the literature. Basic references are de Vaucouleurs et al. (1991) and Young et al. (1989); others are cited in the text.

\begin{tabular}{llccccccccc}
\hline Source & Type & $\begin{array}{c}D \\
{[\mathrm{Mpc}]}\end{array}$ & $\begin{array}{c}\text { beam size } \\
24^{\prime \prime} \hat{=}[\mathrm{pc}]\end{array}$ & $\begin{array}{c}p a \\
{\left[{ }^{\circ}\right]}\end{array}$ & $\begin{array}{c}\text { Incl. } \\
{\left[{ }^{\circ}\right]}\end{array}$ & $\begin{array}{c}D_{25} \\
{\left[^{\prime}\right]}\end{array}$ & $\begin{array}{c}\text { IR color } \\
S_{60} / S_{100}\end{array}$ & $\begin{array}{c}L_{\mathrm{IR}} \\
{\left[\times 10^{9} L_{\odot}\right]}\end{array}$ & $\begin{array}{c}L_{\mathrm{IR}} / L_{\mathrm{B}} \\
{\left[\times 10^{9} M_{\odot}\right]}\end{array}$ & $M_{\mathrm{H} \text { I }}$ \\
\hline NGC 253 & SAB(s)c & 2.5 & 290 & $52^{\circ}$ & $78^{\circ}$ & 27.5 & 0.66 & 15.10 & 1.42 & 2.57 \\
NGC 278 & SAB(rs)b & 12.4 & 1440 & $55^{\circ}$ & $?$ & 2.1 & 0.63 & 8.73 & 0.88 & 1.16 \\
NGC 891 & SA(s)b & 9.6 & 1120 & $23^{\circ}$ & $88^{\circ}$ & 13.5 & 0.41 & 19.32 & 1.16 & 3.73 \\
Maffei 2 & SAB(rs)bc & 5.0 & 580 & $26^{\circ}$ & $66^{\circ}$ & $1 ?$ & - & - & - & - \\
IC 342 & SAB(rs)cd & 4.5 & 520 & $25^{\circ}$ & $25^{\circ}$ & 21.4 & 0.46 & 2.26 & 0.62 & 18.2 \\
NGC 2146 & SB(s)ab pec & 17.0 & 1980 & $135^{\circ}$ & $65^{\circ}$ & 6.0 & 0.83 & 85.74 & 3.10 & 8.49 \\
M 82 & I0 & 3.2 & 370 & $70^{\circ}$ & $81^{\circ}$ & 11.2 & 1.02 & 29.74 & 5.74 & 1.30 \\
NGC 3628 & SB pec & 6.7 & 780 & $104^{\circ}$ & $89^{\circ}$ & 14.8 & 0.48 & 6.31 & 0.55 & 2.81 \\
NGC 4631 & SB(s)d sp & 7.5 & 870 & $82^{\circ}$ & $86^{\circ}$ & 15.5 & 0.53 & 11.90 & 0.56 & 10.1 \\
M 51 & SA(s)bc pec & 9.6 & 1120 & $170^{\circ}$ & $20^{\circ}$ & 11.2 & 0.46 & 30.90 & 0.60 & 4.71 \\
M 83 & SAB(s)c & 4.7 & 550 & $45^{\circ}$ & $24^{\circ}$ & 12.9 & 0.54 & 14.98 & 0.65 & 8.10 \\
NGC 6946 & SAB(rs)cd & 5.2 & 610 & $60^{\circ}$ & $30^{\circ}$ & 11.5 & 0.51 & 10.80 & 0.64 & 5.32 \\
\hline
\end{tabular}

\subsection{Total fluxes}

Since we have obtained extended maps of the observed galaxies, it is possible to calculate the total flux integrated over the mapped region. Since this region contains in any case the central emission peak, some conclusions about the total energy radiated in the $\mathrm{CO}(3-2)$ line are possible.

The total flux is proportional to the velocity-integrated intensity and the mapped area on the sky,

$F_{\mathrm{CO}(3-2)}=\frac{2 k \nu^{2}}{c^{2}} \int I_{\mathrm{CO}(3-2)} \mathrm{d} \Omega$,

and is a measure of the total amount of highly excited CO gas, weighted with the distance. The effect of the latter can be eliminated by comparing the power radiated in the $\mathrm{CO}(3-2)$ line. From the values given in Table 3 , we find some indication that the spiral galaxies whose Hubble type classification includes "peculiar" (NGC 2146, NGC 3628, and M 51; see Col. 2 in Table 2), as well as M 82, show an enhanced output in the $\mathrm{CO}(3-2)$ line. This means that disturbances, e.g. due to encounters with neighbouring galaxies, lead to larger amounts of highly excited molecular gas. Interestingly, this conclusion is not valid for starburst galaxies in general: Maffei 2 and NGC 253 show a similar value of $P_{\mathrm{CO}(3-2)}$ to the normal galaxy NGC 891 or NGC 4631, a "classical" interacting galaxy. Note that it is difficult to compare $P_{\mathrm{CO}(3-2)}$ for NGC 278 with the other objects, since in NGC 278 our map covers the entire galaxy.

We can obtain a different picture when we normalize the power emitted in the $\mathrm{CO}(3-2)$ line to the area from which this power is emitted. Thus we define the frequencyintegrated radiation surface density by

$\Sigma_{\mathrm{CO}(3-2)} \equiv P_{\mathrm{CO}(3-2)} A_{\mathrm{em}}^{-1}$,

where $A_{\mathrm{em}}$ is the galaxy surface area (when looked faceon) from which the $\mathrm{CO}(3-2)$ line is emitted. This area is calculated by $A_{\mathrm{em}}=l_{x}^{2}$ for the edge-on galaxies of our sample $\left(i>60^{\circ}\right)$, and by $A_{\mathrm{em}}=l_{x} l_{y}$ for the other objects, to account for the different orientations.

We find that the two starburst galaxies NGC 253 and M 82 show the highest value of $\Sigma_{\mathrm{CO}(3-2)}$, whereas most other starburst galaxies (including NGC 2146) show values a factor 3 smaller. M 83 can be found somewhere in between. The spiral galaxies NGC 891 and especially M 51 are another considerable factor weaker, despite the fact that only the extent of the central emission peak is used to calculate $\Sigma_{\mathrm{CO}(3-2)}$ in both cases. At the lower end of this scale we find NGC 278, in agreement with e.g. infrared luminosity or H I mass, which point - together with our other results on this object - to NGC 278 being a rather small and quiet galaxy. The "mild starburst" NGC 4631 has an even lower value for $\Sigma_{\mathrm{CO}(3-2)}$ than NGC 278, reflecting also the large size of the area from where $\mathrm{CO}(3-2)$ emission is detected.

\subsection{Line ratios and excitation}

By comparing the measured velocity-integrated intensities $I_{\mathrm{CO}(3-2)}$ with data from the (1-0) and (2-1) transitions, we can calculate line ratios by

$R_{3,1}=I_{\mathrm{CO}(3-2)} / I_{\mathrm{CO}(1-0)}$

(and $R_{3,2}$ analog). We have made fully sampled maps of the galaxies; in addition the spatial resolution of our observations allows us to determine line ratios for the disks and the nuclei of the galaxies separately.

The line ratios we obtained are given in Table 3. Care must be taken when the data for the various transitions are observed with different resolutions: in this case the process of calculating line ratios introduces a larger error (e.g. for M 83). In addition, many authors don't give error estimates at all. Thus the errors given in Table 3 
Table 3. Source properties as obtained from the $\mathrm{CO}(3-2)$ data. In Cols. 2 to 6 values are given for the central emission peak. The source extent along the major and minor axis is given as measured size (in ", Col. 4) and already deconvolved by a Gaussian beam with $H P B W=24^{\prime \prime}$ (in kpc, Col. 5). See text for further details.

\begin{tabular}{|c|c|c|c|c|c|c|c|}
\hline Source & $\begin{array}{c}F_{\mathrm{CO}(3-2)} \\
{\left[\times 10^{4} \mathrm{Jy} \mathrm{km} \mathrm{s}^{-1}\right]}\end{array}$ & $\begin{array}{l}P_{\mathrm{CO}(3-2)} \\
{\left[\times 10^{30} \mathrm{~W}\right]}\end{array}$ & $\begin{array}{c}\Theta_{x} \times \Theta_{y} \\
{\left[{ }^{\prime \prime} \times{ }^{\prime \prime}\right]}\end{array}$ & $\begin{array}{c}l_{x} \times l_{y} \\
{[\mathrm{kpc} \times \mathrm{kpc}]}\end{array}$ & $\begin{array}{c}\Sigma_{\mathrm{CO}(3-2)} \\
{\left[\times 10^{30} \mathrm{~W} \mathrm{kpc}^{-2}\right]}\end{array}$ & $\begin{array}{c}\text { line ratios } \\
R_{3,1}(\text { centre } / \text { disk })\end{array}$ & $\begin{array}{l}\Delta V_{\text {centre }} \\
{\left[\mathrm{km} \mathrm{s}^{-1}\right]}\end{array}$ \\
\hline NGC 253 & $8.74 \pm 0.30$ & $6.0 \pm 0.2$ & $43^{\prime \prime} \times 28^{\prime \prime}$ & $0.43 \times 0.18 \pm 0.03$ & $32.4 \pm 3.7$ & $(0.8 / 0.5) \pm 0.1$ & 200 \\
\hline NGC 278 & $0.37 \pm 0.03$ & $6.2 \pm 0.5$ & $45^{\prime \prime} \times 46^{\prime \prime}$ & $2.71 \times 2.77 \pm 0.24$ & $0.83 \pm 0.14$ & $0.8 \pm 0.2$ & 40 \\
\hline NGC 891 & $0.47 \pm 0.06$ & $4.7 \pm 0.6$ & $30^{\prime \prime} \times 25^{\prime \prime}$ & $0.80 \times 0.36 \pm 0.10$ & $7.3 \pm 2.0$ & $(0.4 / 0.5) \pm 0.1$ & 100 \\
\hline Maffei 2 & $1.87 \pm 0.08$ & $5.1 \pm 0.3$ & $40^{\prime \prime} \times 32^{\prime \prime}$ & $0.78 \times 0.52 \pm 0.05$ & $8.4 \pm 1.5$ & $(1.3 / 0.8) \pm 0.2$ & 150 \\
\hline IC 342 & $2.19 \pm 0.06$ & $4.9 \pm 0.2$ & $45^{\prime \prime} \times 34^{\prime \prime}$ & $0.84 \times 0.52 \pm 0.05$ & $11.2 \pm 1.5$ & $(1.3 / 1.0) \pm 0.1$ & 60 \\
\hline NGC 2146 & $1.94 \pm 0.14$ & $61.4 \pm 4.5$ & $37^{\prime \prime} \times 29^{\prime \prime}$ & $2.36 \times 1.36 \pm 0.17$ & $11.0 \pm 1.6$ & $(1.3 / 1.0) \pm 0.2$ & 280 \\
\hline M 82 & $14.63 \pm 0.40$ & $16.4 \pm 0.5$ & $52^{\prime \prime} \times 33^{\prime \prime}$ & $0.71 \times 0.36 \pm 0.04$ & $32.5 \pm 3.1$ & $(1.0 / 0.8) \pm 0.2$ & 230 \\
\hline NGC 3628 & $2.68 \pm 0.14$ & $13.1 \pm 0.7$ & $41^{\prime \prime} \times 29^{\prime \prime}$ & $1.09 \times 0.50 \pm 0.07$ & $11.0 \pm 1.3$ & $(1.4 / 1.0) \pm 0.2$ & 220 \\
\hline NGC 4631 & $1.35 \pm 0.11$ & $8.3 \pm 0.7$ & $100^{\prime \prime} \times 32^{\prime \prime}$ & $3.63 \times 0.77 \pm 0.15$ & $0.63 \pm 0.07$ & $(1.0 / 0.7) \pm 0.2$ & 80 \\
\hline M 51 & $1.60 \pm 0.20$ & $16.1 \pm 2.0$ & $53^{\prime \prime} \times 80^{\prime \prime}$ & $2.21 \times 3.55 \pm 0.10$ & $2.05 \pm 0.25$ & $(0.5 / 0.7) \pm 0.2$ & 130 \\
\hline M 83 & $3.12 \pm 0.15$ & $7.5 \pm 0.4$ & $35^{\prime \prime} \times 33^{\prime \prime}$ & $0.58 \times 0.52 \pm 0.05$ & $24.9 \pm 3.9$ & $(1.4 / 0.9) \pm 0.3$ & 100 \\
\hline NGC 6946 & $1.42 \pm 0.07$ & $4.2 \pm 0.2$ & $31^{\prime \prime} \times 32^{\prime \prime}$ & $0.48 \times 0.53 \pm 0.06$ & $16.5 \pm 3.3$ & $(1.3 / 1.0) \pm 0.2$ & 150 \\
\hline
\end{tabular}

mainly reflect the errors in the measured $\mathrm{CO}(3-2)$ intensities and only relative errors in the used $\mathrm{CO}(1-0)$ data sets. However, any systematical and calibration errors in the used $\mathrm{CO}(1-0)$ data affect both the centre and disk values for the line ratios.

The values we find for $R_{3,1}$ cover the range from 0.4 (in the centre of NGC 891) up to 1.4 (in the centre of NGC 3628 and M 83). In localized regions within the galaxies the ratios may be even higher, but they cannot be measured with limited spatial resolution. We should also note that the observed values are distance-dependent, since our telescope beam corresponds to different linear scales for the different galaxies.

When comparing the obtained line ratios with other parameters, we find that there is no obvious trend with any other quantity. All galaxies classified as starbursts show $R_{3,1}=1.2-1.4$ in the centre, except M 82 and NGC 253 with values of 1.0 and 0.8 , respectively (note, however, the uncertain calibration for NGC 253 as mentioned in Sect. 3.1). Two galaxies show an exceptionally low ratio: NGC 891 and M 51. Both are usually considered as relatively normal galaxies without a nuclear starburst, but with ongoing star formation in the spiral arms.

\subsection{Summary and outlook}

We have obtained extended maps of the $\mathrm{CO}(3-2)$ emission in twelve nearby galaxies, using the Heinrich-HertzTelescope on Mt. Graham, Arizona. Our observations were not restricted to starburst galaxies, but include also "normal" spiral galaxies. Further not only the central positions of the objects have been observed, as in earlier studies. Hence for the first time we were able to estimate spatially integrated parameters of the $\mathrm{CO}(3-2)$ emission in the observed objects, to measure the extent of the emission, and to investigate variations within these objects.

We find that the $\mathrm{CO}(3-2)$ emission in the observed galaxies is not confined to the nucleus. In some cases the $\mathrm{CO}(3-2)$ is as extended as the $\mathrm{CO}(1-0)$ emission. Nevertheless, the $\mathrm{CO}(3-2)$ is more concentrated to star forming structures (nuclear regions and spiral arms), which is shown by the decrease of the $\mathrm{CO}(3-2) / \mathrm{CO}(1-0)$ line ratios within the galaxies from the very centre to regions further out. The $\mathrm{CO}(3-2)$ luminosity is enhanced in objects which contain a nuclear starburst or morphological peculiarities. The power emitted in the $\mathrm{CO}(3-2)$ line from the central regions is highest in the starburst galaxies NGC 2146, M 82, NGC 3628, and in the spiral galaxy M 51. When normalized to the size of the emission region, the $\mathrm{CO}(3-2)$ emission is a factor 3 higher in the starbursts NGC 253 and M 82 than in most other objects. With the present spatial resolution, the line ratios $R_{3,1}$ seem to be independent of Hubble type, color, or luminosity. Most galaxies with enhanced central star formation show line ratios of $R_{3,1} \sim 1.3$ in the very centre and $\sim 1.0$ at a radius of about $1 \mathrm{kpc}$. Those objects with a ring-like molecular gas distribution (NGC 253, M 82, and NGC 4631) show lower ratios. The two galaxies that have $\mathrm{CO}(3-2)$ emission distributed over their spiral arms (NGC 891 and M 51) show very low line ratios $(\sim 0.5)$. Further our data suggest that even if starbursts are localized phenomena, they imply different properties of the molecular gas also in the disks of the host galaxies. This is shown by line ratios that are systematically higher in the disks of starburst galaxies than in those of normal ones.

These observations have shown that it is necessary to study several types of galaxies in order to obtain results which are not biased towards starburst objects. Further it is not sufficient to observe only one point per galaxy, since 
this cannot account for differences in the $\mathrm{CO}(3-2)$ spatial distribution.

In order to improve this study, we will survey more galaxies in the future. This will lead to better statistics of the findings presented here. Even higher transitions of ${ }^{12} \mathrm{CO}$ will be included, as well as observations of other isotopomers to restrict interpretations concerning optical depth effects.

Acknowledgements. We thank R. Beck, W. Huchtmeier, and R. Kothes for their collaboration during the observations. Further we thank the SMTO staff and D. Muders for their help at the telescope, and T. Wilson for his comments on the manuscript. M.D. acknowledges financial support by a joint CNRS/MPG stipendium, and C.N. acknowledges support by the Studienstiftung des Deutschen Volkes.

\section{References}

Allen, R. J., Baldwin, J. E., \& Sancisi, R. 1978, A\&A, 62, 397 Armus, L., Heckman, T. M., Weaver, K. A., \& Lehnert, M. D. 1995, ApJ, 445, 666

Baars, J. W. M., \& Martin, R. N. 1996, Rev. Mod. Astr., 9, Astr. Ges. Hamburg, 111

Baars, J. W. M., Martin, R. N., Mangum, J. G., McMullin, J. P., \& Peters, W. L. 1999, PASP, 111, 627

Ball, L., \& Kirk, J. G. 1995, A\&A, 303, L57

Braine, J., Combes, F., Casoli, F., et al. 1993, A\&AS, 97, 887

Boissé, P., Casoli, F., \& Combes, F. 1987, A\&A, 173, 229

Casoli, F., Clausset, F., Combes, F., Viallefond, F., \& Boulanger, F. 1990, A\&A, 233, 357

Combes, F. 1978, A\&A, 65, 47

Comte, G. 1981, A\&AS, 44, 441

de Vaucouleurs, G. 1975, in Galaxies and the Universe, Stars and Stellar Systems 9, ed. A. Sandage, M. Sandage, \& J. Kristian (University of Chicago Press), 309

de Vaucouleurs, G. 1979, ApJ, 227, 729

de Vaucouleurs, G., de Vaucouleurs, A., Corwin, H. G. Jr., et al. 1991, Third reference catalogue of bright galaxies (Springer-Verlag, New York)

Ferguson, A. M. N., Wyse, R. F. G., Gallagher, J. S., \& Hunter, D. A. 1998, ApJ, 506, L19

García-Burillo, S., \& Guélin, M. 1995, A\&A, 299, 657

García-Burillo, S., Guélin, M., Cernicharo, J., \& Dahlem, M. 1992, A\&A, 266, 21

García-Burillo, S., Guélin, M., \& Cernicharo, J. 1993, A\&A, 274,123

Golla, G., Dettmar, R.-J., \& Domgörgen, H. 1996, A\&A, 313, 439

Golla, G., \& Hummel, E. 1994, A\&A, 284, 777

Golla, G., \& Wielebinski, R. 1994, A\&A, 286, 733

Greve, A., Neininger, N., Tarchi, A., \& Sievers, A. 2000, A\&A, 364,409

Güsten, R., Serabyn, E., Kasemann, C., et al. 1993, ApJ, 402, 537

Handa, T., Nakai, N., Sofue, Y., Hayashi, M., \& Fujimoto, M. 1990, PASJ, 42, 1

Harrison, A., Henkel, Ch., \& Russell, A. 1999, MNRAS, 303, 157

Ho, P. T. P., Turner, J. L., \& Martin, R. N. 1987, ApJ, 322, L67

Houghton, S., Whiteoak, J. B., Koribalski, B., et al. 1997, A\&A, 325, 923
Hummel, E., Beck, R., \& Dahlem, M. 1991, A\&A, 248, 23

Hurt, R. L., \& Turner, J. L. 1991, ApJ, 377, 434

Hurt, R. L., Turner, J. L., Ho, P. T. P., \& Martin, R. N. 1993, ApJ, 404, 602

Hüttemeister, S., Henkel, C., Mauersberger, R., et al. 1995, A\&A, 295, 571

Irwin, J. A., \& Sofue, Y. 1996, ApJ, 464, 738

Ishizuki, S., Kawabe, R., Ishiguro, M., et al. 1990, Nature, 344, 224

Israel, F. P., White, G. J., \& Baas, F. 1995, A\&A, 302, 343

Jackson, J. M., \& Ho, P. T. P. 1988, ApJ, 324, L5

Knapp, G. R., Phillips, T. G., Huggins, P. J., Leighton, R. B., \& Wannier, P. G. 1980, ApJ, 240, 60

Lo, K. Y., Stephens, S., Rosenthal, E., Eales, S., \& WynnWilliams, C. G. 1990, in Submillimeter Astronomy, ed. G. D. Watt, \& A. S. Webster (Kluwer, Dordrecht)

Mao, R. Q., Henkel, C., Schulz, A., et al. 2000, A\&A, 358, 433

Mauersberger, R., Henkel, C., Wielebinski, R., Wiklind, T., \& Reuter, H.-P. 1996, A\&A, 305, 421

Mauersberger, R., Henkel, C., Walsh, W., \& Schulz, A. 1999, A\&A, 341, 256

McCall, M. L. 1989, AJ, 79, 1341

Nakai, N., Kuno, N., Handa, T., \& Sofue, Y. 1994, PASJ, 46, 527

Neininger, N., \& Dumke, M. 1999, Proc. Natl. Acad. Sci. USA, 96,5360

Neininger, N., Guélin, M., Klein, U., García-Burillo, S., \& Wielebinski, R. 1998, A\&A, 339, 737

Nieten, Ch., Dumke, M., Beck, R., \& Wielebinski, R. 1999, A\&A, 347, L5

Pence, W. D. 1981, ApJ, 247, 473

Petitpas, G. R., \& Wilson, C. D. 1998, ApJ, 503, 219

Rand, R. J. 1994, A\&A, 285, 833

Reuter, H.-P., Krause, M., Wielebinski, R., \& Lesch, H. 1991, A\&A, 248, 12

Rickard, L. J., Turner, B. E., \& Palmer, P. 1977, ApJ, 218, L51

Rieke, G. H., Lebofsky, M. J., Thompson, E. I., Low, F. J., \& Tokunaga, A. T. 1980, ApJ, 238, 24

Rieke, G., Lebofsky, M., \& Walker, C. 1988, ApJ, 325, 679

Rhoads, J. E. 1998, AJ, 115, 472

Rogstad, D. H., \& Shostak, G. S. 1972, ApJ, 176, 315

Rots, A. H. 1978, AJ, 83, 219

Rupen, M. P. 1991, AJ, 102, 48

Sandage, A., \& Tammann, G. A. 1974, ApJ, 194, 559

Sandage, A., \& Tammann, G. A. 1975, ApJ, 196, 313

Sandage, A., \& Tammann, G. A. 1981, Revised ShapleyAmes Catalog of Bright Galaxies (Carnegie Institution of Washington)

Sargent, A., Sutton, E., Masson, R., Lo, K., \& Phillips, T. 1985, ApJ, 289, 150

Schmidt, A. A., Bica, E., \& Alloin, D. 1990, MNRAS, 243, 620

Schulz, A., Güsten R., Köster, B., \& Krause, D. 2001, A\&A, in press

Scoville, N. Z., Thakkar, D., Carlstrom, J. E., \& Sargent, A. I. 1993, ApJ, 404, L59

Sofue, Y., Doi, M., \& Ishizuki, S. 1988, PASJ, 40, 511

Spinrad, H., Bahcall, J., Becklin, E. E., et al. 1971, ApJ, 163, L25

Stanek, K. Z., Knapp, G. R., Young, K., \& Phillips, T. G. 1995, ApJS, 100, 169

Steppe, H., Mauersberger, R., Schulz, A., \& Baars, J. W. M. 1990, A\&A, 233, 410

Talbot, R. J. Jr., Jensen, E. B., \& Dufour, R. J. 1979, ApJ, 229, 91 
Tammann, G. A., \& Sandage, A. R. 1968, ApJ, 151, 825

Tilanus, R. P. J., Tacconi, L. J., Sutton, E. C., et al. 1991, ApJ, 376, 500

Tully, R. B. 1988, Nearby Galaxies Catalogue (Cambridge University Press)

Turner, J. L., \& Ho, P. T. P. 1983, ApJ, 268, L79

Turner, J. L., Martin, R. N., \& Ho, P. T. P. 1990, ApJ, 351, 418

Wall, W. F., Jaffe, D. T., Israel, F. P., \& Bash, F. N. 1991, ApJ, 380,384

Weiß, A., Neininger, N., Hüttemeister, S., \& Klein, U. 2001, A\&A, 365, 571

Weliachew, L., Casoli, F., \& Combes, F. 1988, A\&A, 199, 29

White, G. J., Ellison, B., Claude, S., Dent, W. R. F., \& Matheson, D. N. 1994, A\&A, 284, L23
Wielebinski, R., Dumke, M., \& Nieten, Ch. 1999, A\&A, 347, 634

Wild, W., Eckart, A., Genzel, R., et al. 1990, in The Interstellar Medium in External Galaxies: Summaries of Contributed Papers, ed. D. J. Hollenbach, \& H. A. Thronson (NASA CP 3084)

Wilson, T. L., Muders, D., Butner, H. M., et al. 2000, in Science with the Atacama Large Millimeter Array, ed. A. Wootten, ASP Conf. Ser., in press

Young, J. S., \& Scoville, N. 1982, ApJ, 258, 467

Young, J. S., Xie, S., Kenney, J. D. P., \& Rice, W. L. 1989, ApJS, 70, 699

Young, J. S., Xie, S., Tacconi, L., et al. 1995, ApJS, 98, 219

Xie, S., Young, J., \& Schloerb, F. P. 1994, ApJ, 421, 434 